\title{
Finite temperature and quench dynamics in the Transverse Field Ising Model from form factor expansions
}

\author{
Etienne Granet $^{1 \star}$, Maurizio Fagotti ${ }^{2}$ and Fabian H. L. Essler ${ }^{1}$,
}

1 The Rudolf Peierls Centre for Theoretical Physics, Oxford University, Oxford OX1 3PU, UK

2 LPTMS, CNRS, Université Paris Sud, Université Paris-Saclay, 91405 Orsay, France

* etienne.granet@physics.ox.ac.uk

\begin{abstract}
We consider the problems of calculating the dynamical order parameter twopoint function at finite temperatures and the one-point function after a quantum quench in the transverse field Ising chain. Both of these can be expressed in terms of form factor sums in the basis of physical excitations of the model. We develop a general framework for carrying out these sums based on a decomposition of form factors into partial fractions, which leads to a factorization of the multiple sums and permits them to be evaluated asymptotically. This naturally leads to systematic low density expansions. At late times these expansions can be summed to all orders by means of a determinant representation. Our method has a natural generalization to semi-local operators in interacting integrable models.
\end{abstract}

\section{Contents}

\begin{tabular}{lll}
\hline & Introduction & 3
\end{tabular}

$\begin{array}{lll}1.1 & \text { Finite temperature dynamics } & 3\end{array}$

\begin{tabular}{lll}
\hline 1.2 & Quench dynamics & 4
\end{tabular}

\begin{tabular}{lll}
\hline 1.3 & Local vs semi-local operators & 5
\end{tabular}

\begin{tabular}{|lll}
\hline 1.4 & One and two-point functions of semi-local operators & 6
\end{tabular}

$\begin{array}{lll}2 & \text { Transverse Field Ising Model } & 6\end{array}$

$\begin{array}{lll}2.1 & \text { Quenches in the quench action framework } & 7\end{array}$

\begin{tabular}{|lll}
\hline 2.2 & Dynamical correlation functions at finite temperature & 8
\end{tabular}

3 Systematic approach to form factor expansions for semi-local operators 8

$\begin{array}{lll}3.1 & \text { Case } h<1 \text { : identical number of particles } & 9\end{array}$

$\begin{array}{lll}3.1 .1 & \text { Partial fraction decomposition of form factors } & 9\end{array}$

\begin{tabular}{lll}
\hline 3.1 .2 & Carrying the sum over the momenta $p_{i}$ & 10
\end{tabular}

\begin{tabular}{lll}
\hline 3.1 .3 & Constraints on the functions $f$ & 11
\end{tabular}

\begin{tabular}{lll}
\hline 3.1 .4 & Example: correlation function at $\mathcal{O}\left(\rho_{\beta}\right)$ uniformly in $t$ at large $t$ & 12
\end{tabular}

3.1.5 Example: correlation function at $\mathcal{O}\left(\rho_{\beta}^{2}\right)$ uniformly in $t$ at large $t \quad 13$

\begin{tabular}{llll}
\hline 3.1 .6 & Recursive structure of $A\left(I_{0}, I_{1}, I_{2}\right)$ & 14
\end{tabular}

$\begin{array}{lll}3.1 .7 & \text { Partial fraction in } A\left(I_{0}, I_{1}, I_{2}\right) \text { leading in density } & 14\end{array}$

3.1.8 Result: correlation function at $\mathcal{O}\left(\rho_{\beta}^{2}\right)$ uniformly in $t$ at large $t \quad 15$

\begin{tabular}{lll}
\hline 3.2 & Case $h>1$ : different numbers of particles & 17
\end{tabular}

$\begin{array}{lll}3.2 .1 & \text { General structure } & 17\end{array}$ 
$\begin{array}{lll}3.2 .2 & \text { Case } M=N-1 & 17\end{array}$

$\begin{array}{lll}3.2 .3 & \text { Case } M=N+1 \text { and } k=1 & 18\end{array}$

$\begin{array}{lll}3.2 .4 & \text { Case } M=N+1 \text { and } k=0 & 19\end{array}$

3.2 .5 Result: correlation functions for $h>1$ at leading order in $\rho_{\beta} \quad 19$

$\begin{array}{lll}3.3 & \text { Case } h=1 & 20\end{array}$

$\begin{array}{lll}3.4 & \text { Quantum quench case } & 20\end{array}$

$\begin{array}{lll}3.4 .1 & \text { Generalities } & 20\end{array}$

$\begin{array}{lll}3.4 .2 & \text { Differences to the finite temperature case } & 20\end{array}$

3.4.3 Result: $\mathcal{O}\left(\rho_{Q}^{2}\right)$ uniformly in $t$ at large $t \quad 21$

3.5 Comments on the form factor summation 22

$\begin{array}{lll}3.5 .1 & \text { Which states govern the late time dynamics? } & 22\end{array}$

3.5.2 Non-vanishing low-density limit of form factors 23

\begin{tabular}{lll}
\hline 3.5 .3 & Static correlations & 24
\end{tabular}

\begin{tabular}{|ll|}
4 & Quantum quench dynamics beyond low densities \\
\hline
\end{tabular}

$\begin{array}{lll}4.1 & \text { Determinant representation } & 25\end{array}$

$\begin{array}{lll}4.1 .1 & \text { Approximation } & 25\end{array}$

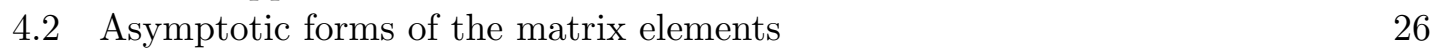

\begin{tabular}{|ll}
\hline 4.2 .1 & Diagonal matrix elements
\end{tabular}

\begin{tabular}{lll}
\hline \hline 4.2 .2 & Off-diagonal matrix elements & 26
\end{tabular}

\begin{tabular}{|ll}
\hline 4.2 .3 & Approximate determinant representation \\
\hline
\end{tabular}

\begin{tabular}{|ll}
4.3 & Evaluating the determinant \\
\hline & 27
\end{tabular}

\begin{tabular}{lll}
\hline 4.3 .1 & First order & 27
\end{tabular}

\begin{tabular}{lll}
\hline 4.3 .2 & Second order & 27
\end{tabular}

\begin{tabular}{|lll}
4.3 .3 & Leading late-time contribution at all orders & 28
\end{tabular}

\begin{tabular}{lll}
\hline 4.3 .4 & Influence of the boundaries 28
\end{tabular}

4.4 Result: late-time asymptotics of the order parameter after a quench 29

\begin{tabular}{|lll}
\hline 4.5 & Numerical Checks & 29
\end{tabular}

5 Dynamical correlation functions at arbitrary finite temperatures $\quad 30$

5.1 Ordered phase $h<1 \quad 30$

5.1 .1 Two-point dynamical correlation functions in the ordered phase 31

\begin{tabular}{lll}
\hline 5.1 .2 & Numerical checks & 32
\end{tabular}

$\begin{array}{lll}5.2 & \text { Disordered phase } h>1 & 34\end{array}$

5.2.1 An approximate mapping to the ordered case 34

\begin{tabular}{|ll}
5.2 .2 & Two-point dynamical correlation functions in the disordered phase 35
\end{tabular}

\begin{tabular}{lll}
\hline 5.2 .3 & Numerical checks & 35
\end{tabular}

\begin{tabular}{lll}
\hline 6 & Summary and Discussion & 37
\end{tabular}

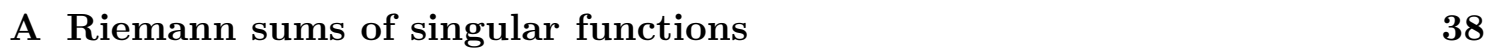

\begin{tabular}{lll}
\hline A.1 Oscillatory sums & 38
\end{tabular}

\begin{tabular}{lll}
\hline A.1.1 Generic example & 38
\end{tabular}

\begin{tabular}{|lll}
\hline \hline A.1.2 & Elementary oscillatory sums with singularities & 39
\end{tabular}

\begin{tabular}{ll|r}
\hline A.1.3 & Equations (43) & 39
\end{tabular}

\begin{tabular}{llr}
\hline A.1.4 & Equations (86) & 40 \\
\hline A.1.5 & Two-(19n
\end{tabular}

\begin{tabular}{|lll}
\hline A.1.5 & Two-dimensional sums & 41
\end{tabular}

\begin{tabular}{|ll}
\hline A.2 & Non-oscillatory sums \\
\hline
\end{tabular}

\begin{tabular}{lll}
\hline A.2.1 & Generic example & 42
\end{tabular}

\begin{tabular}{lll}
\hline A.2.2 & Two-dimensional sums & 42
\end{tabular} 


\section{Introduction}

As a consequence of the existence of extensive numbers of conservation laws with local densities the dynamical properties of quantum integrable models at finite energy densities are both rich and unusual. The two main settings of interest are finite temperature equilibrium response and time evolution after quantum quenches. In the first setting the aim is to determine two-point functions of the form

$$
\chi_{\mathcal{A B}}(x, t)=\frac{1}{Z(\beta)} \operatorname{Tr}\left[e^{-\beta H} \mathcal{A}(x, t) \mathcal{B}(0,0)\right],
$$

where $Z(\beta)=\operatorname{Tr}\left(e^{-\beta H}\right)$ and $\mathcal{A}(x, t)$ is a Heisenberg picture operator, while in the quench setting one is interested in equal time expectation values

$$
\langle\Psi|\mathcal{O}(x, t)| \Psi\rangle,
$$

where $|\Psi\rangle$ is an initial state that is a linear superposition of an exponentially (in system size) large number of energy eigenstates.

\subsection{Finite temperature dynamics}

Early work on determining (1) focussed on the spin- $1 / 2 \mathrm{XY}$ chain in a magnetic field, which can be mapped to a non-interacting model of free fermions [1]. In [2,3 it was shown that two-point functions fulfil systems of nonlinear differential equations, which in the transverse-field Ising limit can be efficiently solved numerically [4. The dynamics at the Ising critical point was obtained in [5,6. The long time and distance asymptotics of two-point functions in the XX limit was obtained from the solution of a Riemann-Hilbert problem in 7 arising from a Fredholm determinant representation 8, 9. A semiclassical approach to the low temperature regime in interacting integrable models was pioneered in 10 and has proved very useful [11 13] due to its relative simplicity. It is however limited in that it applies only to very low temperatures and cannot be easily extended. Perhaps the most direct approach to evaluating (1) or (2) is by introducing spectral representations, e.g.

$$
\chi_{\mathcal{A B}}(\ell, t)=\frac{1}{Z(\beta)} \sum_{n, m} e^{-\beta E_{n}}\langle n|\mathcal{A}(0,0)| m\rangle\langle m|\mathcal{B}(0,0)| n\rangle e^{i t\left(E_{n}-E_{m}\right)-i \ell\left(P_{n}-P_{m}\right)},
$$

where $|n\rangle$ are eigenstates of energy $E_{n}$ and momentum $P_{n}$. Early investigations of (3) focussed on integrable quantum field theories in the infinite volume 14 19, where the spectral representations need to be regularized. This problem was solved in $20-22$ and a systematic low temperature expansion of dynamical two point functions in Fourier space was obtained [22 25]. For some correlators this expansion exhibits divergences close to the zero temperature mass shell and needs to be summed to all orders. Carrying out this summation exactly is an open problem. A similar approach was formulated for the case of the Ising field theory in [26 and used to obtain the late-time asymptotic behaviour of the order parameter two-point function [27]. 
In order to go beyond the low temperature regime in interacting integrable models it is useful to work in the micro-canonical ensemble and employ typicality ideas. This provides a more efficient spectral representation of the form

$$
\chi_{\mathcal{A} \mathcal{B}}(\ell, t)=\sum_{m}\left\langle E_{\beta}|\mathcal{A}(0,0)| m\right\rangle\left\langle m|\mathcal{B}(0,0)| E_{\beta}\right\rangle e^{i t\left(E_{\beta}-E_{m}\right)-i \ell\left(P_{\beta}-P_{m}\right)},
$$

where $E_{\beta}$ is a typical energy eigenstate at the energy density corresponding to inverse temperature $\beta$ [28]. The representation (4) can be analyzed numerically for finite systems 28. Moreover, in particular limiting cases it appears to be very efficient in that only a small number of states need to be summed over [29]. Very recently an axiomatic approach to formulating form factors between states at finite energy densities in the infinite volume limit was proposed [30] and used to formulate a spectral representation. Using this representation to obtain explicit results for dynamical two-point functions remains an open problem.

An alternative approach to finite temperature dynamics is based on the Quantum Transfer Matrix approach 31, 32. The latter is highly efficient for determining static properties [33 36] and can be extended to dynamical correlation functions [37. Very recently this method has been successfully applied to the XX model [38 40] and state-ofthe-art results have been obtained. The generalization to determine dynamical two-point functions in interacting integrable models is an open problem.

The late time asymptotics of certain finite temperature two-point functions can also be accessed by applying generalized hydrodynamics [41,42] to the linear response regime, see [43, 44].

\subsection{Quench dynamics}

Early work on quench dynamics again focussed on models that can be analyzed by means of free fermion techniques [45 53]. Notably, in [50, 51] exact results for the late time behaviour of one and two point functions of the order parameter in the transverse field Ising model (TFIM) after quantum quenches were obtained. One way of going beyond free theories is to employ a spectral representation

$$
\langle\Psi|\mathcal{O}(x, t)| \Psi\rangle=\sum_{n, m}\langle\Psi \mid n\rangle\langle m \mid \Psi\rangle\langle n|\mathcal{O}(0,0)| m\rangle e^{i\left(E_{n}-E_{m}\right) t-i\left(P_{n}-P_{m}\right) x} .
$$

This was used to obtain the late time behaviour for small quenches in the TFIM [50,51, 54 57] and the sine-Gordon model [58 60]. The small quench regime is also accessible by semiclassical methods 61 65, which have the advantage of being significantly simpler to implement. A much more efficient spectral representation is provided by the Quench Action Approach 66. For translationally invariant initial states this allows one to express expectation values of local operators after a quantum quench from an initial state $|\Psi\rangle$ as

$$
\lim _{L \rightarrow \infty}\langle\mathcal{O}(t)\rangle=\lim _{L \rightarrow \infty}\left(\frac{\left\langle\Psi|\mathcal{O}(t)| \Phi_{s}\right\rangle}{2\left\langle\Psi \mid \Phi_{s}\right\rangle}+\frac{\left\langle\Phi_{s}|\mathcal{O}(t)| \Psi\right\rangle}{2\left\langle\Phi_{s} \mid \Psi\right\rangle}\right)
$$

where $\left|\Phi_{s}\right\rangle$ is a representative state fixed by two requirements: first, it is a simultaneous eigenstate of the Hamiltonian and of the (quasi)local conservation laws $I^{(n)}$ of the theory under consideration, and, second, it correctly reproduces the expectation values

$$
\lim _{L \rightarrow \infty} \frac{\left\langle\Psi\left|I^{(n)}\right| \Psi\right\rangle}{L}=\lim _{L \rightarrow \infty} \frac{\left\langle\Phi_{s}\left|I^{(n)}\right| \Phi_{s}\right\rangle}{L} .
$$


Expression (7) affords a more efficient spectral representation involving only a single sum over energy eigenstates as

$$
\left\langle\Psi|\mathcal{O}(t)| \Phi_{s}\right\rangle=\sum_{n}\langle\Psi \mid n\rangle\left\langle n|\mathcal{O}(0)| \Phi_{s}\right\rangle e^{i t\left(E_{n}-E_{s}\right)} .
$$

The behaviour in the steady state reached in the limit $t \rightarrow \infty$ is given by the expectation value in the representative state and this has been analyzed in a number of cases 7075 . The time dependence is significantly more difficult to obtain. So far results are restricted to a particular one-point function for small quenches in the sine-Gordon model [58] and density correlations at late times after a quench in the repulsive Lieb-Linger model [76].

\subsection{Local vs semi-local operators}

Locality properties of the operator of interest have important implications in both finite temperature and quench contexts. For quantum quenches this was emphasized in [48, 49] and clarified through explicit calculations in Refs [50, 51, 58, 66]. A precise definition of the mutual locality index $\omega(A, B)$ of two operators exists in the context of relativistic integrable quantum field theory, see e.g. 77]; specifically, the product of operators $A(x, \tau) B(0,0)$ as a function of $(x, \tau)$ has the property

$$
\mathcal{A}_{C}[A(x, \tau) B(0,0)]=e^{2 \pi i \omega(A, B)} A(x, \tau) B(0,0),
$$

where $\mathcal{A}_{C}$ denotes the analytic continuation along a counter-clockwise contour $C$ around zero. Let us for simplicity consider the case of a diagonal scattering theory with only a single "elementary" particle excitation created by the field $\Psi(x)$. A convenient basis of energy eigenstates is given in terms of scattering states of elementary excitations

$$
\left|\theta_{1}, \ldots, \theta_{n}\right\rangle
$$

where $\theta_{j}$ are rapidity variables related to the energy and momentum of a single-particle excitation by $\epsilon(\theta)=M \cosh (\theta), p(\theta)=\frac{M}{v} \sinh (\theta)$. Spectral representations of correlation functions (in the infinite volume) involve form factors like

$$
\left\langle\theta_{1}, \ldots, \theta_{N}|A(0,0)| \theta_{1}^{\prime}, \ldots, \theta_{M}^{\prime}\right\rangle .
$$

As we will see below the case $M=N$ is of particular interest. Local operators have vanishing mutual locality index with $\Psi(x)$. As a consequence of kinematic poles 78 the form factors become singular when rapidities in the set $\left\{\theta_{j}\right\}$ approach those in $\left\{\overline{\theta_{j}^{\prime}}\right\}$. In the case $N=M$ the structure of singularities is 21

$$
\begin{aligned}
\left\langle\theta_{1}+\epsilon_{1}, \ldots, \theta_{N}+\epsilon_{N}|A(0,0)| \theta_{1}, \ldots, \theta_{N}\right\rangle= & \sum_{i_{N}=1}^{N} \ldots \sum_{i_{1}=1}^{N} a_{i 1 \ldots i_{N}}\left(\theta_{1}, \ldots, \theta_{N}\right) \frac{\epsilon_{i_{1}} \ldots \epsilon_{i_{N}}}{\epsilon_{1} \ldots \epsilon_{N}} \\
& +\ldots
\end{aligned}
$$

In contrast, for semi-local operators $B$ with $\omega(B, \Psi)=1$ one has instead

$$
\left\langle\theta_{1}+\epsilon_{1}, \ldots, \theta_{N}+\epsilon_{N}|A(0,0)| \theta_{1}, \ldots, \theta_{N}\right\rangle=\frac{2^{N}\langle 0|A(0,0)| 0\rangle}{\epsilon_{1} \ldots \epsilon_{N}}+\ldots
$$

This shows that form factors of such semi-local operators are much more singular than the ones for local operators. Form factors in integrable lattice models have analogous structures of singularities. 


\subsection{One and two-point functions of semi-local operators}

The nature of singularities for semi-local operators $(13)$ has been exploited previously to obtain results for 1-point functions after small quantum quenches [58, 66]. The aim of this work is to extend this approach to general quantum quenches as well as to dynamical two-point functions at finite temperatures. We focus on the case of the order parameter in the TFIM because the form factors are particularly simple in this case. This allows us to exhibit in considerable detail which states in the respective spectral representations contribute to the late time asymptotics of one and two-point functions. These considerations can be generalized to interacting integrable models, as will be shown in a following publication.

\section{Transverse Field Ising Model}

The TFIM Hamiltonian on a ring with $L$ sites reads

$$
H(h)=-J \sum_{j=1}^{L}\left(\sigma_{j}^{x} \sigma_{j+1}^{x}+h \sigma_{j}^{z}\right)
$$

where $\sigma_{j}^{\alpha}$ acts like the corresponding Pauli matrix at sites $j$ and like the identity elsewhere. We assume $J, h>0$ and consider periodic boundary conditions. We refer the reader to appendix A of [50] for details about the diagonalization of this Hamiltonian. We simply recall here that it can be expressed in terms of free fermions $\alpha_{k}$ as

$$
H(h)=\sum_{k} \varepsilon(k)\left(\alpha_{k}^{\dagger} \alpha_{k}-\frac{1}{2}\right), \quad \varepsilon(k)=2 J \sqrt{1+h^{2}-2 h \cos k},
$$

and that the Hilbert space is divided into a Neveu Schwartz (NS) sector with states of the form

$$
\left|p_{1} \ldots p_{2 n}\right\rangle=\alpha_{p_{1}}^{\dagger} \ldots \alpha_{p_{2 n}}^{\dagger}|0\rangle_{\mathrm{NS}}, \quad p_{i}=\frac{2 \pi}{L}\left(n_{i}+\frac{1}{2}\right), \quad n_{i}=-\frac{L}{2}, \ldots, \frac{L}{2}-1
$$

and a Ramond (R) sector

$$
\left|k_{1} \ldots k_{2 m+1}\right\rangle=\alpha_{k_{1}}^{\dagger} \ldots \alpha_{k_{2 m+1}}^{\dagger}|0\rangle_{\mathrm{R}}, \quad k_{i}=\frac{2 \pi n_{i}}{L} \quad n_{i}=-\frac{L}{2}, \ldots, \frac{L}{2}-1,
$$

where the energy $E\left(\left\{p_{i}\right\}\right)$ and momentum $P\left(\left\{p_{i}\right\}\right)$ of such states are given by

$$
E\left(\left\{p_{i}\right\}\right)=\sum_{i=1}^{2 n} \varepsilon\left(p_{i}\right), \quad P\left(\left\{p_{i}\right\}\right)=\sum_{i=1}^{2 n} p_{i}
$$

with an identical relation for the Ramond sector.

We will be interested in two problems involving the summation of form factors of the order parameter over the full Hilbert space, which are given by 80.83

$$
\begin{aligned}
& \mathrm{NS}\left\langle q_{1}, \ldots, q_{2 n}\left|\sigma_{l}^{x}\right| p_{1} \ldots p_{m}\right\rangle_{\mathrm{R}}=e^{-i l\left(\sum_{j=1}^{2 n} q_{j}-\sum_{l=1}^{m} p_{m}\right)} i^{n+m / 2}\left(4 J^{2} h\right)^{(m-2 n)^{2} / 4} \sqrt{\xi \xi_{T}} \\
\times & \prod_{j=1}^{2 n}\left(\frac{e^{\eta_{q_{j}}}}{L \varepsilon\left(q_{j}\right)}\right)^{1 / 2} \prod_{l=1}^{m}\left(\frac{e^{-\eta_{p_{l}}}}{L \varepsilon\left(p_{l}\right)}\right)^{1 / 2} \prod_{j<j^{\prime}}^{2 n} \frac{\sin \frac{q_{j}-q_{j^{\prime}}}{2}}{\varepsilon_{q_{j} q_{j^{\prime}}}} \prod_{l<l^{\prime}}^{m} \frac{\sin \frac{p_{l}-p_{l^{\prime}}}{2}}{\varepsilon_{p_{l} p_{l^{\prime}}}} \prod_{j=1}^{2 n} \prod_{l=1}^{m} \frac{\varepsilon_{q_{j} p_{l}}}{\sin \frac{q_{j}-p_{l}}{2}} .
\end{aligned}
$$


Here $\xi=\left|1-h^{2}\right|^{1 / 4}, m$ is even (odd) for $h<1(h>1)$, and

$$
\varepsilon_{a b}=\frac{\varepsilon(a)+\varepsilon(b)}{2} .
$$

The terms $\xi_{L}$ and $\eta_{k}$ do not depend on the momenta (except $k$ ) and for large $L$ approach one with exponential accuracy

$$
\xi_{L} \approx 1, \quad e^{\eta_{k}} \approx 1
$$

thus, they will be set to 1 in the following.

\subsection{Quenches in the quench action framework}

We consider the following quantum quench setup [50,51]: at time $t=0$ we prepare the system in the ground state of the TFIM (14) at a magnetic field $h_{0}<1$

$$
|\Psi\rangle=\left|0 ; h_{0}\right\rangle_{\mathrm{NS}} .
$$

At times $t>0$ we evolve the system with Hamiltonian $H(h)$ with $h_{0} \neq h<1$. As $|\Psi\rangle$ is not an eigenstate of $H(h)$ this results in interesting dynamics. The order parameter one-point function at time $t>0$ is given by (6), where the representative state $\left|\Phi_{s}\right\rangle$ is characterized by the root density 66$]$

$$
\begin{aligned}
\rho(k) & =\frac{1-\cos \Delta_{k}}{4 \pi}, \\
\cos \Delta_{k} & =\frac{h h_{0}-\left(h+h_{0}\right) \cos k+1}{\sqrt{1+h^{2}-2 h \cos k} \sqrt{1+h_{0}^{2}-2 h_{0} \cos k}} .
\end{aligned}
$$

For later convenience we define the density of particles in the representative state

$$
\rho_{Q}=\int_{-\pi}^{\pi} d k \rho(k)
$$

In a large finite volume $L$ we may choose 66

$$
\left|\Phi_{s}\right\rangle=\left|q_{1},-q_{1}, \ldots, q_{N},-q_{N}\right\rangle_{\mathrm{NS}},
$$

where the momenta $q_{i}$ are distributed according to the root density $\rho(k)$. The time-evolved initial state is given by

$$
|\psi(t)\rangle=\prod_{p>0} \frac{1+i e^{-2 i t \varepsilon(p)} K(p) \alpha_{-p}^{\dagger} \alpha_{p}^{\dagger}}{\sqrt{1+K^{2}(p)}}|0\rangle,
$$

with $K(p)=\tan \left(\Delta_{p} / 2\right)$.

Eqn (6) thus provides the following representation for the order parameter one-point function

$$
\begin{aligned}
\left\langle\sigma_{\ell}^{x}(t)\right\rangle=\operatorname{Re}[ & \sum_{M=0}^{\infty} \frac{(-1)^{M}}{M !} \sum_{\substack{0<p_{1}, \ldots, p_{M} \\
\in \mathrm{R}}} \mathrm{R}\left\langle p_{1},-p_{1}, \ldots, p_{M},-p_{M}\left|\sigma_{\ell}^{x}\right| q_{1},-q_{1}, \ldots, q_{N},-q_{N}\right\rangle_{\mathrm{NS}} \\
& \left.\times \prod_{j=1}^{N} \frac{\sqrt{1+K\left(q_{j}\right)^{2}}}{K\left(q_{j}\right)} e^{-2 i t \varepsilon\left(q_{j}\right)} \prod_{j=1}^{M} \frac{K\left(p_{j}\right)}{\sqrt{1+K\left(p_{j}\right)^{2}}} e^{2 i t \varepsilon\left(p_{j}\right)}\right],
\end{aligned}
$$

that is a sum of form factors over states that are expressed in terms of pairs of momenta. 


\subsection{Dynamical correlation functions at finite temperature}

In the TFIM, the density of momenta $q$ of the representative state $\left|E_{\beta}\right\rangle$ in (4) is

$$
\rho(q)=\frac{1}{2 \pi} \frac{1}{1+e^{\beta \varepsilon(q)}} .
$$

It is very useful in the following to define the corresponding density

$$
\rho_{\beta}=\int_{-\pi}^{\pi} d q \rho(q)
$$

In practice a representative state is constructed from $\rho(q)$ as follows. We first construct the particle counting function $z(q)$ by integrating the root density

$$
z(q)=\int_{-\pi}^{q} d p \rho(p) .
$$

We then solve the equations

$$
z\left(q_{j}^{(0)}\right)=\frac{2 \pi j}{L}, \quad j=1, \ldots, N,
$$

where $N$ is fixed by the requirement that $\left|q_{j}^{(0)}\right| \leq \pi$. Finally we set

$$
q_{j}=\frac{2 \pi}{L}\left(\left\lfloor\frac{L}{2 \pi} q_{j}^{(0)}-\frac{1}{2}\right\rfloor+\frac{1}{2}\right), \quad j=1, \ldots, N .
$$

Inserting a resolution of the identity between the two spin operators in (4) leads to the following spectral representation

$$
\chi^{x x}(\ell, t)=\sum_{M=0}^{+\infty} \frac{1}{M !} \sum_{p_{1}, \ldots, p_{M}}\left|\left\langle p_{1}, \ldots, p_{M}\left|\sigma_{l}^{x}\right| q_{1}, \ldots, q_{N}\right\rangle\right|^{2} e^{i t(E(\{q\})-E(\{p\}))+i \ell(P(\{p\})-P(\{q\}))}
$$

The terms in the sum depend on the regime of the TFIM: in the ordered phase, $h<1$, $M$ has the same (even/odd) parity as $N$, whereas in the disordered phase, $h>1$, it has opposite parity. Moreover, in contrast to the quench case, (33) involves squares of form factors, and the intermediate states do not have a structure where momenta only appear in pairs $\left\{-p_{i}, p_{i}\right\}$.

\section{Systematic approach to form factor expansions for semi- local operators}

In this section we present a general framework for carrying out the form factor sums (27) and (33) analytically at late times $J t \gg 1$. It is based on decomposing the form factors (19) into partial fractions so that the sums over the $p$ 's decouple and can be evaluated exactly. The key observation is then that an oscillatory sum with a pole of order $d$ like $\sum_{n} \frac{e^{i n t}}{(n+1 / 2)^{d}}$ grows as $t^{d-1}$, so that the leading poles give the leading time behaviour, and the terms in the partial fraction decomposition can be organized according to the total number of poles. This naturally leads to an expansion in the number of particles per unit site $-N / L$ - in the representative state, which has already been proven very 
efficient for simpler quantities such as the free energy in Bethe ansatz solvable interacting models 67,68.

In sections 3.1 and 3.2 we consider the application of this framework in the context of the finite temperature case (33), due to the more canonical sum over form factors that it involves. Since the form factors differ for $h<1, h>1$ and $h=1$, we will treat these cases separately.

We will be interested in large time or space asymptotics of correlation functions, generically defined as requiring the phase it $(E(\{q\})-E(\{p\}))+i l(P(\{p\})-P(\{q\}))$ in (33) to be large. This is in particular the case of the large time and distance asymptotics at fixed

$$
\alpha=\frac{t}{\ell},
$$

on which we will focus. However, the static correlations case $t=0$ and large $l$ is also covered by our calculations; we refer the reader to section 3.5 .3 for details on this case. For later convenience we introduce the following notations

$$
\begin{aligned}
& \bar{\varepsilon}(x)=\varepsilon(x)-\frac{x}{\alpha}, \\
& v_{\max }=\max _{|x| \leq \pi} \varepsilon^{\prime}(x),
\end{aligned}
$$

where $v_{\max }$ is the maximal group velocity of the elementary fermion excitations in the TFIM. According to whether there exists an $x_{0}$ such that $\bar{\varepsilon}^{\prime}\left(x_{0}\right)=0$ ('time-like region', $t v_{\max }>\ell$ for $t, \ell \geq 0$ ) or not ('space-like region', $t v_{\max }<\ell$ for $t, \ell \geq 0$ ), the next-toleading terms in our expansions differ. We will in the following compute these terms in the space-like region. Their calculation in the time-like region is more involved and will be reported elsewhere.

In section 3.4 we briefly present the application of our framework to the dynamics after quantum quenches.

\subsection{Case $h<1$ : identical number of particles}

In this subsection we treat the case $h<1$, for which the sum 33 includes intermediate states with the same number $N$ of particles as the representative state. We will show in section 3.2 that the contributions of intermediate states with different particle numbers $M \neq N$ is always subleading in time. We exploit this result right away and focus on states with $M=N$ in this subsection.

\subsubsection{Partial fraction decomposition of form factors}

We recall that the partial fraction decomposition of a ratio of two polynomials $\frac{P(X)}{\prod_{i}\left(X-x_{i}\right)^{a_{i}}}$ with distinct $x_{i}$ 's is the writing

$$
\frac{P(X)}{\prod_{i}\left(X-x_{i}\right)^{a_{i}}}=P_{0}(X)+\sum_{i} \sum_{\nu=1}^{a_{i}} \frac{B_{i, \nu}}{\left(X-x_{i}\right)^{\nu}},
$$

with $P_{0}(X)$ a polynomial of degree $\operatorname{deg}(P)-\sum_{i} a_{i}$ and $B_{i, \nu}$ independent of $X$, given by $B_{i, \nu}=\left.\frac{1}{\left(a_{i}-\nu\right) !}\left(\frac{d}{d X}\right)^{a_{i}-\nu}\left(P(X)\left(X-x_{i}\right)^{a_{i}}\right)\right|_{X=x_{i}}$.

The squared form factor appearing in 33 can be written as

$$
\left.\left.\right|_{\mathrm{NS}}\left\langle q_{1}, \ldots, q_{N}\left|\sigma_{l}^{x}\right| p_{1}, \ldots, p_{N}\right\rangle_{\mathrm{R}}\right|^{2}=\frac{\xi}{L^{2 N}} F_{\left\{p_{i}\right\}}^{\left\{q_{i}\right\}}
$$


with 1

$$
F_{U}^{V}=\frac{\left|\prod_{u \neq u^{\prime} \in U} \sin \left(\frac{u-u^{\prime}}{2}\right) \prod_{v \neq v^{\prime} \in V} \sin \left(\frac{v-v^{\prime}}{2}\right)\right|}{\prod_{u \neq v \in U, V} \sin ^{2}\left(\frac{u-v}{2}\right)} \frac{\prod_{u, v \in U, V} \varepsilon_{u v}^{2}}{\prod_{u, u^{\prime} \in U} \varepsilon_{u u^{\prime}} \prod_{v, v^{\prime} \in V} \varepsilon_{v v^{\prime}}} .
$$

The $\varepsilon\left(p_{1}\right)$ factors are not polynomial in $p_{1}$ but are nevertheless bounded and without zeros. Seen as a function of $p_{1}$, it can thus be written $\sum_{i} \frac{A_{i}}{\sin ^{2}\left(\frac{p_{1}-q_{i}}{2}\right)}+\frac{B_{i}}{\sin \left(\frac{p_{1}-q_{i}}{2}\right)}+C$ with $A_{i}, B_{i}$ independent of $p_{1}$ and $C$ a bounded function of $p_{1}$. Repeating the operation for the other momenta, one can write

$$
\left.\left.\right|_{\mathrm{NS}}\left\langle q_{1}, \ldots, q_{N}\left|\sigma_{l}^{x}\right| p_{1}, \ldots, p_{N}\right\rangle_{\mathrm{R}}\right|^{2}=\frac{\xi}{L^{2 N}} \sum_{\nu_{1}, \ldots, \nu_{N}=0}^{2} \sum_{\left\{f_{\vec{\nu}}\right\}} \frac{\mathcal{A}\left(\{q\},\{p\},\{\nu\}, f_{\vec{\nu}}\right)}{\prod_{j=1}^{N} \sin ^{\nu_{j}}\left(\frac{p_{j}-q_{f_{\vec{\nu}}(j)}}{2}\right)}
$$

where the second sum is over a complete set of functions $f_{\vec{\nu}}:\left\{i \in\{1, \ldots, N\} \mid \nu_{i} \neq 0\right\} \mapsto$ $\{1, \ldots, N\}$, and where $\mathcal{A}\left(\{q\},\{p\},\{\nu\}, f_{\vec{\nu}}\right)$ is a bounded function of $p_{j}$ if $\nu_{j}=0$, and independent of $p_{j}$ otherwise. In Fig. 3.1.1 we show examples of such functions $f_{\vec{\nu}}$. The important feature of (39) is that each $p$ appears at most once (however, the $q$ 's may appear several times).
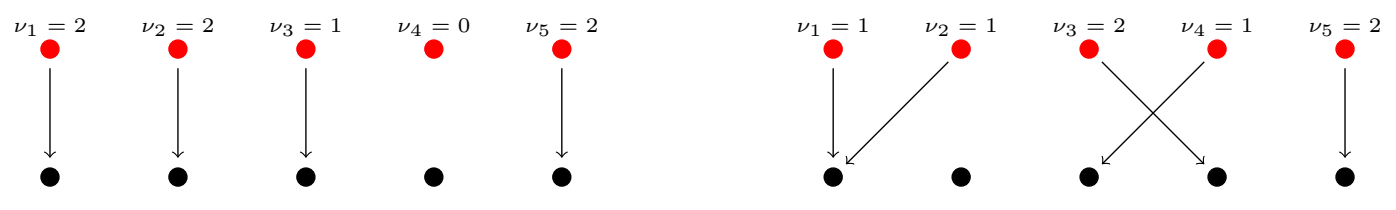

Figure 1: Sketch of two examples of a function $f$ from $p$ 's in red to $q$ 's in black.

\subsubsection{Carrying the sum over the momenta $p_{i}$}

Let us briefly anticipate the method that we will use to carry out the the sum over $p$ in (33). If there is a $\nu_{i}=0$ then the sum over $p_{i}$ is an oscillatory Riemann sum of a bounded function, hence it decays to zero with time. Thus the leading behaviour is obtained for $\nu_{i}>0$ for all $i$. Then (and only in this case) the coefficients $\mathcal{A}\left(\{q\},\{p\},\{\nu\}, f_{\vec{\nu}}\right)$ are independent of the $p$ 's and the maps will no longer depend on $\left\{\nu_{j}\right\}$. These coefficients will be denoted by $A(\{q\},\{\nu\}, f)$ and are obtained as

$$
A(\{q\},\{\nu\}, f)=\left.\left[\left(\prod_{j}\left(2 \frac{d}{d p_{j}}\right)^{2-\nu_{j}} \sin ^{2}\left(\frac{p_{j}-q_{f(j)}}{2}\right)\right) F_{\left\{p_{i}\right\}_{i=1, \ldots, N}}^{\left\{q_{i=1, \ldots, N}\right.}\right]\right|_{\left\{p_{j}=q_{f(j)}\right\}} .
$$

This follows from the partial fraction decomposition, with a factor 2 because of the $1 / 2$ inside the sin.

\footnotetext{
${ }^{1}$ In $F_{U}^{V}$ the sets $U$ and $V$ can contain arbitrary momenta and are not meant to be each in the sectors $R$ and $N S$ (this freedom will be indeed useful in section 3.1.6 below). For this reason we impose in the denominator that the momenta are different $u \neq v$, which is automatically satisfied if they are in different sectors, but not otherwise.
} 
From here on we will only consider terms in the partial fraction decomposition such that $\nu_{i}>0$ for all $i$, so that (40) applies. We denote the corresponding contribution to the spectral representation (33) of $\chi^{x x}(\ell, t)$ by $S$

$$
\begin{array}{r}
S=\frac{\xi}{N ! L^{2 N}} \sum_{p_{1}, \ldots, p_{N}}\left\{\left[\sum_{\nu_{1}, \ldots, \nu_{N}=1}^{2} \sum_{\{f\}} \frac{A(\{q\},\{\nu\}, f)}{\prod_{j=1}^{N} \sin ^{\nu_{j}}\left(\frac{p_{j}-q_{f(j)}}{2}\right)}\right]\right. \\
\left.\times e^{i t(E(\{q\})-E(\{p\}))+i \ell(P(\{p\})-P(\{q\}))}\right\},
\end{array}
$$

where the third sum is over a complete set of functions $f:\{1, \ldots, N\} \mapsto\{1, \ldots, N\}$.

In this form one can perform the sums over $p_{j}$ using the following relations proven in Appendix A

$$
\begin{aligned}
& \chi_{1}(q) \equiv \sum_{p \in \mathrm{R}} \frac{e^{-i t(\bar{\varepsilon}(p)-\bar{\varepsilon}(q))}}{L \sin \left(\frac{p-q}{2}\right)}=-i \operatorname{sgn}\left(t \bar{\varepsilon}^{\prime}(q)\right)+\mathcal{O}\left(L^{0} t^{-1 / 2}\right) \\
& \chi_{2}(q) \equiv \sum_{p \in \mathrm{R}} \frac{e^{-i t(\bar{\varepsilon}(p)-\bar{\varepsilon}(q))}}{L^{2} \sin ^{2}\left(\frac{p-q}{2}\right)}=1-\frac{2\left|t \bar{\varepsilon}^{\prime}(q)\right|}{L}+\mathcal{O}\left(L^{-1} t^{-1 / 2}\right)
\end{aligned}
$$

with $q \in \mathrm{NS}$, to obtain

$$
S=\frac{\xi}{N !} \sum_{\nu_{1}, \ldots, \nu_{N}=1}^{2} \sum_{\{f\}} A(\{q\},\{\nu\}, f) \prod_{i=1}^{N} \frac{\chi_{\nu_{i}}\left(q_{f(i)}\right)}{L^{2-\nu_{i}}} e^{i t\left(\bar{\varepsilon}\left(q_{i}\right)-\bar{\varepsilon}\left(q_{f(i)}\right)\right)} .
$$

Eqns (43) are valid only when $\bar{\varepsilon}^{\prime}(q) \neq 0$. If there is a point where $\bar{\varepsilon}^{\prime}(q)=0$, ie if we are in the time-like region, corrections in time to (43) have to be taken into account, and they are expected to modify significantly the subleading corrections in the correlation function. We leave this matter of discussion for future work.

\subsubsection{Constraints on the functions $f$}

The set of functions $f$ over which we need to sum is actually quite constrained. First, since $F_{\left\{p_{i}\right\}_{i=1, \ldots N}}^{\left\{q_{i}\right\}_{i=1, \ldots N}}=0$ whenever $p_{i}=p_{j}$ we must have $f(i) \neq f(j)$ whenever $\nu_{i}=\nu_{j}=2$.

We also need $f(i) \neq f(j)$ if $\nu_{i}=1$ and $\nu_{j}=2$. Indeed, if $f(i)=f(j)$ then in (40) there is a $\sin ^{2} \frac{p_{i}-p_{j}}{2}$ factor in the numerator of $F_{\left\{p_{n}\right\}_{n=1, \ldots, N}}^{\left\{q_{n}\right\}_{n=1, \ldots, N}}$, but as there is only one derivative with respect to $p_{i}$ and none with respect to $p_{j}$ this factor will make the coefficient $A(\{q\},\{\nu\}, f)$ vanish upon taking $p_{i}=q_{f(i)}=q_{f(j)}=p_{j}$.

More generally, if $f$ takes $k$ times the same value at points with $\nu_{i}=1$, then all the $k(k-1) / 2$ terms $\sin ^{2} \frac{p_{i}-p_{j}}{2}$ contribute to a zero of order $k(k-1)$; since the number of derivatives is equal to $k$, we must have $k=2$.

These arguments show that the sum over $f$ can be replaced by a sum over three disjoint subsets $I_{0}, I_{1}, I_{2} \subset\{1, \ldots, N\}$, where $I_{k}$ is the set of points with $\nu=1$ attained $k$ times by $f$. The remaining points $\{1, \ldots, N\}-\left(I_{0} \cup I_{1} \cup I_{2}\right)$ all have $\nu=2$. There is a combinatorial factor $\frac{N !}{2^{\left|I_{2}\right|}}$ corresponding to the number of such functions with this precise ouput. It 
follows that $A(\{q\},\{\nu\}, f)$ depends only on the sets $I_{0}, I_{1}, I_{2}$ and we have

$$
\begin{aligned}
S=\xi \sum_{\substack{I_{0}, I_{1}, I_{2} \subset\{1, \ldots, N\} \\
\left|I_{0}\right|=\left|I_{2}\right|, \text { all disjoint }}} A\left(I_{0}, I_{1}, I_{2}\right) 2^{-\left|I_{2}\right|} e^{i t\left(\sum_{i \in I_{0}} \bar{\varepsilon}\left(q_{i}\right)-\sum_{i \in I_{2}} \bar{\varepsilon}\left(q_{i}\right)\right)} & \\
& \times \prod_{i \in I_{1}} \frac{\chi_{1}\left(q_{i}\right)}{L} \prod_{i \in I_{2}} \frac{\chi_{1}^{2}\left(q_{i}\right)}{L^{2}} \prod_{i \notin I_{0,1,2}} \chi_{2}\left(q_{i}\right) .
\end{aligned}
$$

The expression for the coefficients $A\left(I_{0}, I_{1}, I_{2}\right)$ can be simplified as follows. We observe that, whenever we have $\nu_{i}=2$ in 40 , the various factors depending on $p_{i}$ and $q_{f(i)}$ precisely compensate one another. Hence we can work with a reduced form factor involving only momenta in $I_{0}, I_{1}, I_{2}$

$$
A\left(I_{0}, I_{1}, I_{2}\right)=\left.\left[\left(\prod_{j=1}^{n+2 m}\left(2 \frac{d}{d p_{j}}\right) \sin ^{2}\left(\frac{p_{j}-q_{f(j)}}{2}\right)\right) F_{\left\{p_{i}\right\}_{i=1, \ldots, n+2 m}}^{\left\{q_{i}\right\}_{i \in I_{0} \cup I_{1} \cup I_{2}}}\right]\right|_{\left\{p_{j}=q_{f(j)}\right\}}
$$

for any function $f$ such that $\{f(i)\}_{i=1, \ldots, n}=I_{1},\{f(i)\}_{i=n+1, \ldots, n+m}=I_{2}$,

and $\{f(i)\}_{i=n+m+1, \ldots, n+2 m}=I_{2}$. The set $I_{2}$ does appear twice by construction, and $I_{0}$ does not appear at all. The decomposition of $\{1, \ldots, n+2 m\}$ into $\{1, \ldots, n\},\{n+1, \ldots, n+m\}$, $\{n+m+1, \ldots, n+2 m\}$ is arbitrary, and it needs only to involve one set with $n$ elements and two sets with $m$ elements.

The sum of all the terms in (45) with $\left|I_{1}\right|=n,\left|I_{0}\right|=\left|I_{2}\right|=m$ will be denoted by $S_{n, 2 m}$. We can factorize $S_{0,0}$ and, using the explicit expression for $\chi_{1}(q)$ and $\chi_{2}(q)$, write

$$
\begin{gathered}
S_{n, 2 m}=\frac{(-i)^{n} S_{0,0}}{(-2)^{m} L^{n+2 m}} \sum_{\substack{I_{0,1,2} \subset\{1, \ldots, N\} \\
\left|I_{0}\right|=\left|I_{2}\right|=m \\
\left|I_{1}\right|=n \\
\text { all disjoint }}} A\left(I_{0}, I_{1}, I_{2}\right) \prod_{\prod_{i \in I_{1}} \operatorname{sgn}\left(t \bar{\varepsilon}^{\prime}\left(q_{i}\right)\right)}\left(1-\frac{2\left|t \bar{\varepsilon}^{\prime}\left(q_{i}\right)\right|}{L}\right) \\
\times e^{i t\left(\sum_{i \in I_{0}} \bar{\varepsilon}\left(q_{i}\right)-\sum_{i \in I_{2}} \bar{\varepsilon}\left(q_{i}\right)\right)} .
\end{gathered}
$$

If $n$ and $m$ stay finite in the limit $L \rightarrow \infty$ we have $\prod_{i \in I_{0,1,2}}\left(1-\frac{2\left|t \bar{\varepsilon}^{\prime}\left(q_{i}\right)\right|}{L}\right)=1+\mathcal{O}\left(L^{-1}\right)$ and hence

$$
\begin{aligned}
S_{n, 2 m}= & \frac{(-i)^{n} S_{0,0}}{(-2)^{m} L^{n+2 m}} \sum_{\substack{q_{1}^{0}<\ldots<q_{m}^{0} \\
q_{1}^{1}<\ldots<q_{n}^{1} \\
q_{1}^{2}<\ldots<q_{m}^{2} \\
\text { all distinct }}} A\left(\left\{q^{0}\right\},\left\{q^{1}\right\},\left\{q^{2}\right\}\right) e^{i t \sum_{i=1}^{m} \bar{\varepsilon}\left(q_{i}^{0}\right)-\bar{\varepsilon}\left(q_{i}^{2}\right)} \prod_{i=1}^{n} \operatorname{sgn}\left(t \bar{\varepsilon}^{\prime}\left(q_{i}^{1}\right)\right) \\
& +\mathcal{O}\left(L^{-1}\right) .
\end{aligned}
$$

Since the momenta selected by the sets $I_{0,1,2}$ are drawn from the momenta $\left\{q_{j} \mid j=\right.$ $1, \ldots, N\}$ of the representative state with density $\rho$, the term $S_{n, 2 m}$ is of order $(N / L)^{n+2 m}$ times $S_{0,0}$. Hence this expansion naturally leads to an expansion in $N / L$.

\subsubsection{Example: correlation function at $\mathcal{O}\left(\rho_{\beta}\right)$ uniformly in $t$ at large $t$}

Let us give some examples. With $I_{0}=I_{1}=I_{2}=\{\}$ we have $A\left(I_{0}, I_{1}, I_{2}\right)=1$ hence the term

$$
S_{0,0}=\xi \prod_{i=1}^{N}\left(1-\frac{2\left|t \bar{\varepsilon}^{\prime}\left(q_{i}\right)\right|}{L}\right)=\xi \exp \left(-2 \int_{-\pi}^{\pi}\left|t \bar{\varepsilon}^{\prime}(x)\right| \rho(x) d x\right)+\mathcal{O}\left(L^{-1}\right),
$$


where, for a function $f(x)$, we used

$$
\lim _{L \rightarrow \infty} \prod_{k=0}^{L-1}\left(1+\frac{f(k / L)}{L}\right)=\exp \int_{0}^{1} f(x) d x .
$$

This term is the correlation function at order 1 in $\rho$ uniformly in large $t$.

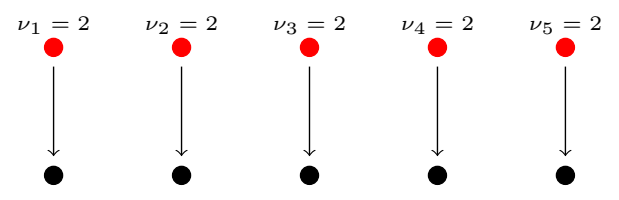

Figure 2: Sketch of configurations contributing to $S_{0,0}$.

\subsubsection{Example: correlation function at $\mathcal{O}\left(\rho_{\beta}^{2}\right)$ uniformly in $t$ at large $t$}

With $I_{1}=\{i, j\}$ and $I_{0}=I_{2}=\{\}$ we have

$$
A\left(I_{0}, I_{1}, I_{2}\right)=\frac{2}{\sin ^{2}\left(\frac{q_{i}-q_{j}}{2}\right)}+\frac{8 \varepsilon^{\prime}\left(q_{i}\right) \varepsilon^{\prime}\left(q_{j}\right)}{\left(\varepsilon\left(q_{i}\right)+\varepsilon\left(q_{j}\right)\right)^{2}} .
$$

This term leads to

$$
S_{2,0}=-\frac{S_{0,0}}{L^{2}} \sum_{i<j}\left(\frac{2}{\sin ^{2}\left(\frac{q_{i}-q_{j}}{2}\right)}+\frac{8 \varepsilon^{\prime}\left(q_{i}\right) \varepsilon^{\prime}\left(q_{j}\right)}{\left(\varepsilon\left(q_{i}\right)+\varepsilon\left(q_{j}\right)\right)^{2}}\right) \operatorname{sgn}\left(\bar{\varepsilon}^{\prime}\left(q_{j}\right) \bar{\varepsilon}^{\prime}\left(q_{i}\right)\right) .
$$

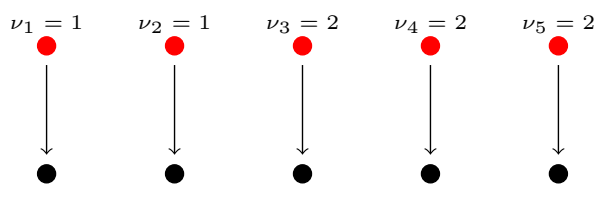

Figure 3: Sketch of configurations contributing to $S_{2,0}$.

For the choice $I_{1}=\{\}$ and $I_{0}=\{i\}, I_{2}=\{j\}$ we have

$$
A\left(I_{0}, I_{1}, I_{2}\right)=-\frac{2}{\sin ^{2}\left(\frac{q_{i}-q_{j}}{2}\right)},
$$

and

$$
S_{0,2}=\frac{S_{0,0}}{L^{2}} \sum_{i \neq j} \frac{e^{i t\left(\bar{\varepsilon}\left(q_{j}\right)-\bar{\varepsilon}\left(q_{i}\right)\right)}}{\sin ^{2}\left(\frac{q_{i}-q_{j}}{2}\right)} \operatorname{sgn}\left(\bar{\varepsilon}^{\prime}\left(q_{j}\right) \bar{\varepsilon}^{\prime}\left(q_{i}\right)\right) .
$$

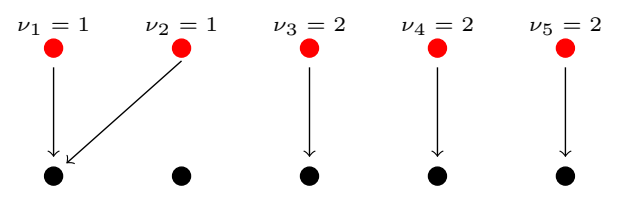

Figure 4: Sketch of configurations contributing to $S_{0,2}$. 
Although they are individually both divergent in $L$ in the scaling limit $L \rightarrow \infty$, their sum is not divergent and is, see appendix $\mathrm{A}$.

$$
S_{2,0}+S_{0,2}=-S_{0,0}\left(4 \pi \int_{-\pi}^{\pi}\left|t \bar{\varepsilon}^{\prime}(x)\right| \rho^{2}(x) d x+c\right),
$$

with the following value in the space-like regime where $\operatorname{sgn}\left(\bar{\varepsilon}^{\prime}(x)\right)$ is constant

$$
c=2 \int_{-\pi}^{\pi} \int_{-\pi}^{\pi} \frac{\rho(y) \rho^{\prime}(x)}{\tan \left(\frac{x-y}{2}\right)} .
$$

Contrarily to the previous case, this order $\rho^{2}$ of the correlation function at fixed large $t$ cannot be uniform in $t$, since it diverges for $t \rightarrow \infty$.

\subsubsection{Recursive structure of $A\left(I_{0}, I_{1}, I_{2}\right)$}

In the general case the amplitudes $A\left(I_{0}, I_{1}, I_{2}\right)$ are obtained from (46), but the sums over momenta associated with the index sets $I_{0}, I_{2}$ in 47 cannot be carried out as simply as in the cases treated above. In fact, as we noted earlier, the derivatives corresponding to $I_{2}, I_{0}$ in 46 have to be applied on the double zero $\sin ^{2} \frac{p_{i}-p_{j}}{2}$ in the numerator to give a non-vanishing result, so that one actually has

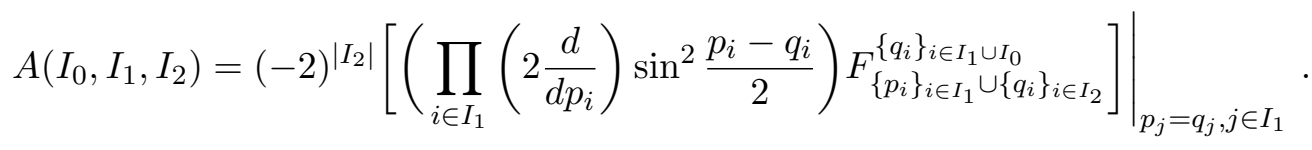

The form factor $F_{\left\{p_{i}\right\}_{i \in I_{1}} \cup\left\{q_{i}\right\}_{i \in I_{2}}}^{\left\{q_{i}\right\}_{i \in I_{1}}}$ can itself be decomposed into partial fractions. One obtains

$$
A\left(I_{0}, I_{1}, I_{2}\right)=(-2)^{\left|I_{2}\right|} \sum_{\substack{\nu_{i} \in\left\{I_{1}, 1,2\right\} \\ i \in I_{1} \cup I_{2} \\ \nu_{i}=1 \text { if } i \in I_{1}}} \sum_{\substack{f:\left\{i \in I_{1} \cup I_{2} / \nu_{i}>0\right\} \\ \forall \rightarrow I_{1} \cup I_{0} \\ f(i)=i \text { if } i \in I_{1}}} \frac{A\left(\left\{q_{i}\right\}_{i \in I_{1} \cup I_{0}},\{\nu\}, f\right)}{\prod_{j \in I_{2}} \sin ^{\nu_{j}} \frac{q_{j}-q_{f(j)}}{2}} .
$$

We observe that the sum over $\left\{q^{2}\right\}$ in (47) will play a similar role to the sum over $p$ 's in (33), with however the important difference that they are drawn from the original $q$ 's of the representative state and are not arbitrary momenta as is the case for the $p$ 's in 33 .

\subsubsection{Partial fraction in $A\left(I_{0}, I_{1}, I_{2}\right)$ leading in density}

Relation (57) reveals a recursive structure in the calculation of $A\left(I_{0}, I_{1}, I_{2}\right)$. However, we will not develop this recursion further here, but will rather focus on the leading partial fractions of (57) obtained with a set $\{\nu\}$ such that $\nu_{i}=2$ for $i \in I_{2}$ and $\nu_{i}=1$ for $i \in I_{1}$, and functions $f: I_{1} \cup I_{2} \mapsto I_{1} \cup I_{0}$ that map $I_{2}$ to $I_{0}$ in a one-to-one fashion and fulfil $f(i)=i$ for $i \in I_{1}$. 


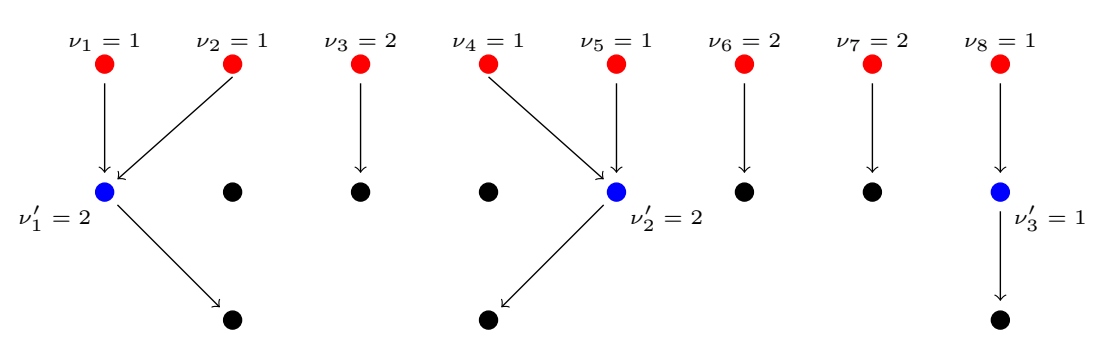

Figure 5: Sketch of the two functions $f$ after one step of recursion. In red are indicated the $p$ 's, in blue the $q$ 's that play the role of the $p$ 's after the first step of the recursion, that are those with index in $I_{1}$ or $I_{2}$. The 'new' $q$ 's on the last row are those with index in $I_{1}$ or $I_{0}$.

The coefficient then reads

$$
A\left(\left\{q_{i}\right\}_{i \in I_{1} \cup I_{0}},\{\nu\}, f\right)=\left.\left[\left(\prod_{i \in I_{1}}\left(2 \frac{d}{d p_{i}}\right) \sin ^{2} \frac{p_{i}-q_{i}}{2}\right) F_{\left\{p_{i}\right\}_{i \in I_{1}}}^{\left\{q_{i}\right\}_{i \in I_{1}}}\right]\right|_{p_{i}=q_{i}, i \in I_{1}} .
$$

Let us select one $i \in I_{1}$ and introduce a reduced set $I_{1}^{\prime}=I_{1}-\{i\}$. Performing the derivative with respect to $p_{i}$ gives

$$
\begin{aligned}
A\left(\left\{q_{i}\right\}_{i \in I_{1} \cup I_{0}},\{\nu\}, f\right) & =\left(\prod_{k \in I_{1}^{\prime}}\left(2 \frac{d}{d p_{k}}\right) \sin ^{2} \frac{p_{k}-q_{k}}{2}\right) \\
& \left.2\left(\sum_{k \in I_{1}^{\prime}} \frac{1}{\tan \frac{q_{i}-p_{k}}{2}}-\frac{\varepsilon^{\prime}\left(q_{i}\right)}{\varepsilon_{q_{i} p_{k}}}-\sum_{k \in I_{1}^{\prime}} \frac{1}{\tan \frac{q_{i}-q_{k}}{2}}-\frac{\varepsilon^{\prime}\left(q_{i}\right)}{\varepsilon_{q_{i} q_{k}}}\right) F_{\left\{p_{k}\right\}_{k \in I_{1}^{\prime}}}^{\left\{q_{k}\right\}_{k \in I_{1}^{\prime}}}\right|_{p_{k}=q_{k}, k \in I_{1}^{\prime}} .
\end{aligned}
$$

We observe that the first factor in the second line has to be differentiated precisely one more time for the result not to vanish (the fact that $f$ is one-to-one on $I_{1}$ to $I_{1}$ is essential for this), so that

$$
\begin{aligned}
A\left(\left\{q_{i}\right\}_{i \in I_{1} \cup I_{0}},\{\nu\}, f\right)=\sum_{j \in I_{1}^{\prime}} & \left(\frac{2}{\sin ^{2} \frac{q_{i}-q_{j}}{2}}+2 \frac{\varepsilon^{\prime}\left(q_{i}\right) \varepsilon^{\prime}\left(q_{j}\right)}{\varepsilon_{q_{i} q_{j}}^{2}}\right) \\
\times & {\left[\left(\prod_{k \in I_{1}^{\prime \prime}}\left(2 \frac{d}{d p_{k}}\right) \sin ^{2} \frac{p_{k}-q_{k}}{2}\right) F_{\left\{p_{k}\right\}_{k \in I_{1}^{\prime \prime}}}^{\left.\left\{q_{k}\right\}_{k \in I_{1}^{\prime \prime}}\right]\left.\right|_{p_{k}=q_{k}, k \in I_{1}^{\prime \prime}},},\right.}
\end{aligned}
$$

with $I_{1}^{\prime \prime}=I_{1}-\{i, j\}$. Applying the same reasoning to the remaining momenta yields

$$
A\left(\left\{q_{i}\right\}_{i \in I_{1} \cup I_{0}},\{\nu\}, f\right)=\sum_{P \text { pairings of } I_{1}} \prod_{(i, j) \in P}\left(\frac{2}{\sin ^{2} \frac{q_{i}-q_{j}}{2}}+8 \frac{\varepsilon^{\prime}\left(q_{i}\right) \varepsilon^{\prime}\left(q_{j}\right)}{\left(\varepsilon\left(q_{i}\right)+\varepsilon\left(q_{j}\right)\right)^{2}}\right) \equiv \phi\left(I_{1}\right) .
$$

\subsubsection{Result: correlation function at $\mathcal{O}\left(\rho_{\beta}^{2}\right)$ uniformly in $t$ at large $t$}

In analogy with our notations for the first level of the recursive structure we denote by $S_{n, 2 m \mid 0,0}$ all contributions to (47) that arise by specifying $\nu_{i}=2$ for $i \in I_{2}$ in (57). We observe that in (56) the form factor vanishes if there are coinciding momenta in $I_{0}$ and in $I_{2}$, or momenta that occur both in $I_{1}$ and $I_{0}$ or $I_{2}$. Hence one only has to impose that 
momenta in $I_{1}$ are distinct among themselves, and that momenta in $I_{0}$ are distinct from those of $I_{2}$. This gives

$$
\begin{aligned}
& S_{n, 2 m \mid 0,0}=\frac{(-i)^{n} S_{0,0}}{n !(m !)^{2} L^{n+2 m}} \times \\
& \sum_{\substack{q_{1}^{1}, \ldots, q_{n}^{1} \\
\text { all distinct }}} \sum_{\substack{q_{1}^{0}, \ldots, q_{m}^{0} \\
q_{1}^{2}, \ldots, q_{m}^{2} \\
q^{0} \text { distinct from } q^{2}}} \phi\left(\left\{q^{1}\right\}\right) \sum_{\begin{array}{c}
f:\left\{q^{2}\right\} \rightarrow\left\{q^{0}\right\} \\
\text { one-to-one }
\end{array}} \frac{e^{i t \sum_{i=1}^{m} \bar{\varepsilon}\left(q_{i}^{0}\right)-\bar{\varepsilon}\left(q_{i}^{2}\right)}}{\prod_{j=1}^{m} \sin ^{2} \frac{q_{j}^{2}-q_{f(j)}^{0}}{2}} \prod_{i=1}^{n} \operatorname{sgn}\left(t \bar{\varepsilon}^{\prime}\left(q_{i}^{1}\right)\right) .
\end{aligned}
$$

The two sets of sums factorize. The first set can be calculated using the results of Appendix A

$$
\begin{aligned}
& \frac{1}{(m !)^{2} L^{2 m}} \sum_{\substack{q_{1}^{0}, \ldots, q_{m}^{0} \\
q_{1}^{2}, \ldots, q_{m}^{2} \\
q^{0} \text { distinct from } q^{2}}} \sum_{\begin{array}{c}
f:\left\{q^{2}\right\} \rightarrow\left\{q^{0}\right\} \\
\text { one-to-one }
\end{array}} \frac{e^{i t \sum_{i=1}^{m} \bar{\varepsilon}\left(q_{i}^{0}\right)-\bar{\varepsilon}\left(q_{i}^{2}\right)}}{\prod_{j=1}^{m} \sin ^{2} \frac{q_{j}^{2}-q_{f(j)}^{0}}{2}}=\frac{1}{m !}\left(\frac{1}{L^{2}} \sum_{q_{i} \neq q_{j}} \frac{e^{i t\left(\bar{\varepsilon}\left(q_{i}\right)-\bar{\varepsilon}\left(q_{j}\right)\right)}}{\sin ^{2} \frac{q_{i}-q_{j}}{2}}\right)^{m} \\
& =\frac{1}{m !}\left(L \frac{4 \pi^{2}}{3} \int_{-\pi}^{\pi} \rho(x)^{3} d x-4 \pi \int_{-\pi}^{\pi} \rho(x)^{2}\left|t \bar{\varepsilon}^{\prime}(x)\right| d x+\mathcal{O}\left(L^{0} t^{-\frac{1}{2}}\right)\right)^{m} .
\end{aligned}
$$

As for the terms in the sum over $\left\{q^{1}\right\}$, they vanish for $n$ odd, while for even $n=2 p$ they are calculated in Appendix $\mathrm{A}$

$$
\begin{aligned}
& \frac{(-i)^{n}}{n ! L^{n}} \sum_{\substack{q_{1}^{1}, \ldots, q_{n}^{1} \\
\text { all distinct }}} \phi\left(\left\{q^{1}\right\}\right) \prod_{i=1}^{n} \operatorname{sgn}\left(t \bar{\varepsilon}^{\prime}\left(q_{i}^{1}\right)\right) \\
& =\frac{(-1)^{p}}{(2 p) !} \frac{(2 p) !}{p ! 2^{p}}\left(\frac{1}{L^{2}} \sum_{q_{i} \neq q_{j}} \operatorname{sgn}\left(\bar{\varepsilon}^{\prime}\left(q_{i}\right) \bar{\varepsilon}^{\prime}\left(q_{j}\right)\right)\left(\frac{2}{\sin ^{2} \frac{q_{i}-q_{j}}{2}}+8 \frac{\varepsilon^{\prime}\left(q_{i}\right) \varepsilon^{\prime}\left(q_{j}\right)}{\left(\varepsilon\left(q_{i}\right)+\varepsilon\left(q_{j}\right)\right)^{2}}\right)\right)^{p} \\
& =\frac{(-1)^{p}}{p !}\left(L \frac{4 \pi^{2}}{3} \int_{-\pi}^{\pi} \rho(x)^{3} d x+c+\mathcal{O}\left(L^{-1}\right)\right)^{p},
\end{aligned}
$$

where $c$ is defined in (55). We note that these two calculations involve the same sums as in $S_{0,2}$ and $S_{2,0}$ above. It follows that the infinite volume limit of the sum of all $S_{n, 2 m \mid 0,0}$ reads

$$
\sum_{n, m \geq 0} S_{n, 2 m \mid 0,0}=S_{0,0} \exp \left(-c-4 \pi \int_{-\pi}^{\pi} \rho(x)^{2}\left|t \bar{\varepsilon}^{\prime}(x)\right| d x+\mathcal{O}\left(t^{-\frac{1}{2}}\right)\right),
$$

where $S_{0,0}$ has been computed in 48 . We have thus obtained the order $\mathcal{O}\left(\rho_{\beta}^{2}\right)$ contribution to the correlation function uniformly in $t$

$$
\chi^{x x}(t, \ell) \approx C \exp \left(-2 \int_{-\pi}^{\pi} \rho(x)(1+2 \pi \rho(x))\left|t \varepsilon^{\prime}(x)-\ell\right| d x\right),
$$

with

$$
C=\xi \exp \left(-2 \int_{-\pi}^{\pi} \int_{-\pi}^{\pi} \frac{\rho(y) \rho^{\prime}(x)}{\tan \left(\frac{x-y}{2}\right)} d x d y\right) .
$$

In the time-like region, the exponent would be the same, but the constant would differ.

This result should be compared to the semiclassical approach of Sachdev and Young [10,79], which gives

$$
\chi_{\mathrm{SY}}^{x x}(t, \ell) \approx \xi \exp \left(-\int_{-\pi}^{\pi} \frac{d k}{\pi} e^{-\beta \varepsilon(k)}\left|t \varepsilon^{\prime}(k)-\ell\right|\right) .
$$

As expected our result reduces to the semiclassical one in the limit $\beta J \gg 1$. 


\subsection{Case $h>1$ : different numbers of particles}

We now turn to the case $h>1$. Here the sum (33) involves only intermediate states with numbers of particles that are different from that of the representative state. Hence we must study form factor sums with $M \neq N$. We will compute the prefactors at order $\mathcal{O}\left(\rho_{\beta}^{1}\right)$ in the space-like region, and because of saddle points effects only at order $\mathcal{O}\left(\rho_{\beta}^{0}\right)$ in the time-like region.

\subsubsection{General structure}

We start by considering the general structure of contributions with $N \neq M$. This discussion applies also to the $h<1$ case and in particular shows that there the dominant contributions arise from $N=M$. As in the case $M=N$, the form factor $\left.\left.\right|_{\mathrm{NS}}\left\langle q_{1}, \ldots, q_{N}\left|\sigma_{l}^{x}\right| p_{1}, \ldots, p_{M}\right\rangle_{\mathrm{R}}\right|^{2}$ can be decomposed into partial fractions

$$
\left|\mathrm{NS}_{\mathrm{S}}\left\langle q_{1}, \ldots, q_{N}\left|\sigma_{l}^{x}\right| p_{1}, \ldots, p_{M}\right\rangle_{\mathrm{R}}\right|^{2}=\frac{\xi(2 J \sqrt{h})^{(M-N)^{2}}}{L^{N+M}} \sum_{\nu_{1}, \ldots, \nu_{M}=0}^{2} \sum_{\left\{f_{\vec{\nu}}\right\}} \frac{A\left(\{q\},\{p\},\{\nu\}, f_{\vec{\nu}}\right)}{\prod_{j=1}^{M} \sin ^{\nu_{j}}\left(\frac{p_{j}-q_{f_{\vec{\nu}}(j)}}{2}\right)}
$$

with $f:\left\{i \in\{1, \ldots, M\} \mid \nu_{i} \neq 0\right\} \mapsto\{1, \ldots, N\}$ any function, and $A\left(\{q\},\{p\},\{\nu\}, f_{\vec{\nu}}\right)$ is a bounded function of $p_{j}$ if $\nu_{j}=0$, and independent of $p_{j}$ otherwise.

The important difference to the case $M=N$ is that we cannot always neglect the contributions with $\nu_{j}=0$. Indeed, let us denote by $k$ the number of $\nu_{j}=0$. The corresponding contributions give rise to $k$ oscillatory bounded integrals that will each decay with time. On the other hand each of the $M-k$ sums over the other momenta $p_{i}$ will generate an oscillating factor $e^{-i t \bar{\varepsilon}\left(q_{f_{\vec{\nu}}(j)}\right)}$ according to $(43)$, while $N$ factors $e^{i t \bar{\varepsilon}\left(q_{k}\right)}$ are already present. The resulting oscillatory sums may have singularities, but according to (43) summing these singularities does not consume any oscillatory factor, it only lowers the number of singularities. Hence in the end we will be left with $|N-M+k|$ oscillatory bounded integrals to perform. In total, there are thus $k+|N-M+k|$ of such integrals. Hence if $M \leq N$ the case $k=0$ is still dominant at late times, but if $M>N$ then all the cases $0 \leq k \leq M-N$ are a priori of the same order. In both cases these leading terms involve $|M-N|$ oscillatory bounded integrals, so that we can conclude that the terms $M \neq N$ are exponentially smaller than the case $M=N$ in the space-like regime, and typically around ${ }^{2} t^{-|M-N| / 2}$ smaller in the time-like regime.

It follows that for $h>1$ the dominant terms in (33) are obtained for $M=N \pm 1$, which we now consider in turn.

\subsubsection{Case $M=N-1$}

For $M=N-1$ the dominant contribution in density in (69) is obtained with $\nu_{i}=2$ for all $i=1, \ldots, N-1$. The function $f:\{1, \ldots, N-1\} \mapsto\{1, \ldots, N\}$ has to be injective so there is one $q_{i}$ not attained by $f$, leaving $(N-1)$ ! possible equivalent choices for $f$ once $q_{i}$ is chosen. Then one has $A\left(\{q\},\{p\},\{\nu\}, f_{\vec{\nu}}\right)=\frac{1}{\varepsilon\left(q_{i}\right)}$. Using 43], the corresponding contribution to 33 is

$$
S_{0,0}^{-}=\frac{2 J \xi \sqrt{h}}{L} \sum_{i=1}^{N} \frac{e^{i t \bar{\varepsilon}\left(q_{i}\right)}}{\varepsilon\left(q_{i}\right)} \prod_{j \neq i} \chi_{2}\left(q_{j}\right)
$$

\footnotetext{
${ }^{2}$ Of course, there are specific degenerate cases where the stationary phase approximation will give a different factor than $\frac{1}{\sqrt{t}}$. Due to the possible time-dependence of the integrand apart from the oscillatory term this factor may also be marginally corrected by logarithms.
} 
This term is of order $\rho$, so in the time-like region it vanishes at the order of our computation. In the space-like region we have

$$
S_{0,0}^{-}=2 J \xi \sqrt{h}\left(\int_{-\pi}^{\pi} \frac{e^{i t \bar{\varepsilon}(x)}}{\varepsilon(x)} \rho(x) d x\right) \exp \left(-2 \int_{-\pi}^{\pi}\left|t \bar{\varepsilon}^{\prime}(x)\right| \rho(x) d x\right) .
$$

There, $\bar{\varepsilon}(x)$ is monotonous and $e^{i t \bar{\varepsilon}(x)}$ is periodic (because the distance $\ell$ is an integer) so the first integral decays with time faster than any power-law and cannot be simplified further.

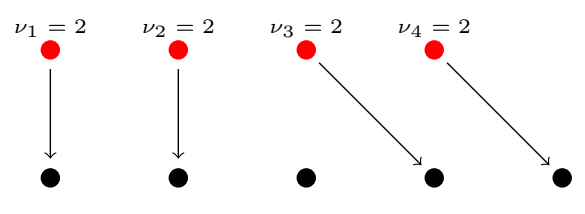

Figure 6: Sketch of the leading configurations contributing to $M=N-1$.

\subsubsection{Case $M=N+1$ and $k=1$}

For $M=N+1$ with one $\nu_{i}=0$, the dominant contribution in 69 is obtained with the remaining $\nu_{j}=2$ for $j \neq i$. Thus $f:\{1, \ldots, N+1\}-\{i\} \mapsto\{1, \ldots, N\}$ has to be one-to-one, leaving $N$ ! equivalent choices. Then one has $A\left(\{q\},\{p\},\{\nu\}, f_{\vec{\nu}}\right)=\frac{1}{\varepsilon\left(p_{i}\right)}$. Using (43), the corresponding contribution to 33 is

$$
S_{0,0}^{+}=\frac{2 J \xi \sqrt{h}}{(N+1) L} \sum_{i=1}^{N+1} \sum_{p_{i}} \frac{e^{-i t \bar{\varepsilon}\left(p_{i}\right)}}{\varepsilon\left(p_{i}\right)} \prod_{j=1}^{N} \chi_{2}\left(q_{j}\right) .
$$

In the infinite volume limit we obtain the following result in the space-like regime

$$
S_{0,0}^{+}=2 J \xi \sqrt{h}\left(\int_{-\pi}^{\pi} \frac{e^{-i t \bar{\varepsilon}(x)}}{2 \pi \varepsilon(x)} d x\right) \exp \left(-2 \int_{-\pi}^{\pi}\left|t \bar{\varepsilon}^{\prime}(x)\right| \rho(x) d x\right),
$$

where the prefactor is accurate to first order in the density $\rho_{\beta}$. In the time-like region the prefactor is only accurate to $\mathcal{O}\left(\rho_{\beta}^{0}\right)$, because saddle point effects arise at order $\mathcal{O}\left(\rho_{\beta}\right)$. A saddle point approximation gives in this regime

$$
S_{0,0}^{+}=\frac{2 J \xi \sqrt{h}}{\sqrt{2 \pi|t|}}\left(\sum_{s \in S P} \frac{e^{\mp i \pi / 4}}{\varepsilon(s) \sqrt{\left|\bar{\varepsilon}^{\prime \prime}(s)\right|}} e^{-i t \bar{\varepsilon}(s)}\right) \exp \left(-2 \int_{-\pi}^{\pi}\left|t \bar{\varepsilon}^{\prime}(x)\right| \rho(x) d x\right),
$$

where $S P=\left\{s, \bar{\varepsilon}^{\prime}(s)=0\right\}$ denotes the set of saddle points and \pm the sign of $t \bar{\varepsilon}^{\prime}(s)$.

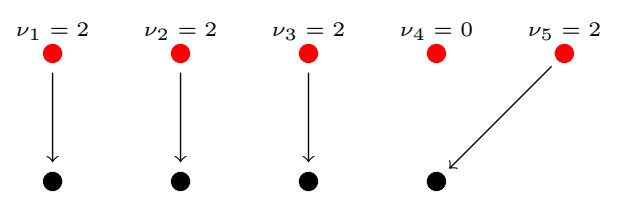

Figure 7: Sketch of the leading configurations contributing to $M=N+1$ and $k=1$. 


\subsubsection{Case $M=N+1$ and $k=0$}

For $M=N+1$ and all $\nu_{i} \neq 0$, the dominant contribution in 69 is obtained by $\{\nu\}, f_{\vec{\nu}}$ such that $\nu_{i}=\nu_{j}=1$ with $f_{\vec{\nu}}(i)=f_{\vec{\nu}}(j)$, and the remaining $\nu_{l}=2$ for $l \neq i, j$. Once $q_{k}=q_{f_{\vec{\nu}}(i)}=q_{f_{\vec{\nu}}(j)}$ are chosen, there are $\left(\begin{array}{c}N+1 \\ 2\end{array}\right) N$ ! possibilities for $f_{\vec{\nu}}$. All of these lead to $A\left(\{q\},\{p\},\{\nu\}, f_{\vec{\nu}}\right)=\frac{-2}{\varepsilon\left(q_{k}\right)}$. Using 43 , the corresponding contribution to 33 becomes

$$
S_{0,2}^{+}=-\frac{2 J \xi \sqrt{h}}{L} \sum_{k=1}^{N} \frac{e^{-i t \bar{\varepsilon}\left(q_{k}\right)}}{\varepsilon\left(q_{k}\right)} \chi_{1}^{2}\left(q_{k}\right) \prod_{j \neq k}^{N} \chi_{2}\left(q_{j}\right) .
$$

Taking the infinite volume limit we obtain

$$
S_{0,2}^{+}=-2 J \xi \sqrt{h}\left(\int_{-\pi}^{\pi} \frac{e^{-i t \bar{\varepsilon}(x)}}{\varepsilon(x)} \chi_{1}^{2}(x) \rho(x) d x\right) \exp \left(-2 \int_{-\pi}^{\pi}\left|t \bar{\varepsilon}^{\prime}(x)\right| \rho(x) d x\right) .
$$

In the space-like region $\chi_{1}(x)=i \operatorname{sgn}\left(\bar{\varepsilon}^{\prime}(x)\right)$ and one has with the prefactor at order $\rho^{1}$

$$
S_{0,2}^{+}=2 J \xi \sqrt{h}\left(\int_{-\pi}^{\pi} \frac{e^{-i t \bar{\varepsilon}(x)}}{\varepsilon(x)} \rho(x) d x\right) \exp \left(-2 \int_{-\pi}^{\pi}\left|t \bar{\varepsilon}^{\prime}(x)\right| \rho(x) d x\right) .
$$

In time-like region, this term vanishes at order $\rho^{0}$.

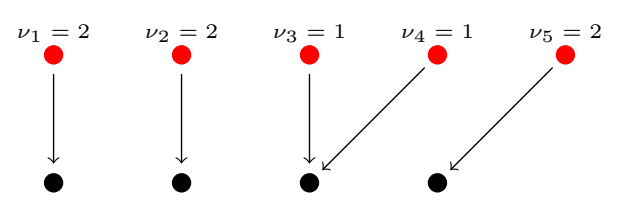

Figure 8: Sketch of the leading configurations contributing to $M=N+1$ and $k=0$.

\subsubsection{Result: correlation functions for $h>1$ at leading order in $\rho_{\beta}$}

Putting everything together, in the space-like regime and at leading order in density we obtain the following result

$$
\chi^{x x}(\ell, t) \approx 2 J \xi \sqrt{h}\left[\int_{-\pi}^{\pi} d x \frac{e^{-i t \bar{\varepsilon}(x)}}{2 \pi \varepsilon(x)}(1+4 \pi \rho(x))\right] \exp \left(-2 \int_{-\pi}^{\pi}\left|t \bar{\varepsilon}^{\prime}(x)\right| \rho(x) d x\right),
$$

In the time-like regime we have instead

$$
\chi^{x x}(\ell, t) \approx \frac{2 J \xi \sqrt{h}}{\sqrt{2 \pi|t|}}\left(\sum_{s \in S P} \frac{e^{\mp i \pi / 4}}{\varepsilon(s) \sqrt{\left|\bar{\varepsilon}^{\prime \prime}(s)\right|}} e^{-i t \bar{\varepsilon}(s)}\right) \exp \left(-2 \int_{-\pi}^{\pi}\left|t \bar{\varepsilon}^{\prime}(x)\right| \rho(x) d x\right),
$$

where the sum is over the saddle points $s$ of $\bar{\varepsilon}(x)$.

This result should be compared to the semiclassical approach of Sachdev and Young [10,79], which gives

$$
\chi_{\mathrm{SY}}^{x x}(t, \ell) \approx 2 J \xi \sqrt{h} \int_{-\pi}^{\pi} \frac{d k}{2 \pi} \frac{e^{-i t \bar{\varepsilon}(x)}}{\varepsilon(x)} \exp \left(-\int_{-\pi}^{\pi} \frac{d k}{\pi} e^{-\beta \varepsilon(k)}\left|t \varepsilon^{\prime}(k)-\ell\right|\right) .
$$

As expected our result reduces to the semiclassical one in the limit $\beta J \gg 1$. 


\subsection{Case $h=1$}

In the case $h=1$ the structure of the form factor (19) is modified compared to the case $h \neq$ 1: since $\varepsilon(0)=0$, there are additional poles. The nature of these poles is moreover different from those appearing in the partial fraction decomposition of the previous sections, since they involve pairs of momenta: for $\epsilon_{p p^{\prime}}$ to vanish we must have $p=p^{\prime}=0$.

We note that at zero temperature the model becomes critical at $h=1$ and correlation functions should exhibit power-law decays. These issues are beyond the scope of this work and will be examined elsewhere.

\subsection{Quantum quench case}

We now turn to the time evolution of the order parameter one-point function after a quench of the transverse field within the ordered phase. Our aim is to evaluate the spectral representation (27) obtained in the framework of the quench action approach.

\subsubsection{Generalities}

The sum over form factors appearing in the context of quantum quench dynamics (27) differs from the finite temperature case (33) notably because now in both states of the form factors the momenta come in pairs $p_{i},-p_{i}$. One can then write

$$
\begin{aligned}
\mathrm{R}\left\langle p_{1},-p_{1}, \ldots, p_{N},-\right. & \left.p_{N}\left|\sigma_{\ell}^{x}\right| q_{1},-q_{1}, \ldots, q_{N},-q_{N}\right\rangle_{\mathrm{NS}}=\frac{(-4)^{N} \sqrt{\xi}}{L^{2 N}} \prod_{j=1}^{N} \sin q_{j} \sin p_{j} \\
\times & \prod_{i, j=1}^{N} \frac{\varepsilon_{q_{j} p_{i}}^{4}}{\varepsilon_{q_{j} q_{i}}^{2} \varepsilon_{p_{j} p_{i}}^{2}} \frac{\prod_{i \neq j=1}^{N}\left(\cos q_{i}-\cos q_{j}\right)\left(\cos p_{i}-\cos p_{j}\right)}{\prod_{i, j=1}^{N}\left(\cos q_{i}-\cos p_{j}\right)^{2}} .
\end{aligned}
$$

Focusing again on $M=N$ in (27), we have

$$
\begin{aligned}
\left\langle\sigma_{\ell}^{x}(t)\right\rangle=\operatorname{Re}\left[\frac{\sqrt{\xi}}{N ! L^{2 N}}\right. & \sum_{\substack{0<p_{1}, \ldots, p_{N} \\
\in \mathrm{R}}} \prod_{j=1}^{N} 4 \frac{f\left(p_{j}\right)}{f\left(q_{j}\right)} \sin p_{j} \sin q_{j} e^{2 i t\left(\varepsilon_{p_{j}}-\varepsilon_{q_{j}}\right)} \\
& \left.\times \prod_{i, j} \frac{\varepsilon_{q_{j} p_{i}}^{4}}{\varepsilon_{q_{j} q_{i}}^{2} \varepsilon_{p_{j} p_{i}}^{2}} \frac{\prod_{i \neq j}\left(\cos q_{i}-\cos q_{j}\right)\left(\cos p_{i}-\cos p_{j}\right)}{\prod_{i, j}\left(\cos q_{i}-\cos p_{j}\right)^{2}}\right],
\end{aligned}
$$

with

$$
f(p) \equiv \frac{K(p)}{\sqrt{1+K^{2}(p)}}=\sqrt{2 \pi \rho(p)} .
$$

\subsubsection{Differences to the finite temperature case}

We apply a partial fraction decomposition to the second line of 82 , seen as a ratio of polynomials in the $\cos p_{j}$. The procedure is the same as in section 3.1 . More precisely, we define

$$
\widetilde{F}_{U}^{V}=\frac{\left|\prod_{u \neq u^{\prime} \in U}\left(\cos u-\cos u^{\prime}\right) \prod_{v \neq v^{\prime} \in V}\left(\cos v-\cos v^{\prime}\right)\right|}{\prod_{u \neq v \in U, V}(\cos u-\cos v)^{2}} \frac{\prod_{u, v \in U, V} \varepsilon_{u v}^{4}}{\prod_{u, u^{\prime} \in U} \varepsilon_{u u^{\prime}}^{2} \prod_{v, v^{\prime} \in V} \varepsilon_{v v^{\prime}}^{2}},
$$


which we decompose into partial fractions with $\cos u$ taken to be the relevant variables. This gives

$$
\widetilde{F}_{\left\{p_{i}\right\}}^{\left\{q_{i}\right\}}=\sum_{\nu_{1}, \ldots, \nu_{N}=0}^{2} \sum_{\left\{f_{\vec{\nu}}\right\}} \frac{\mathcal{A}\left(\{q\},\{p\},\{\nu\}, f_{\vec{\nu}}\right)}{\prod_{j=1}^{N}\left(\cos p_{j}-\cos q_{f_{\vec{\nu}}(j)}\right)^{\nu_{j}}},
$$

where the second sum is over any function $f:\left\{i \in\{1, \ldots, N\} \mid \nu_{i} \neq 0\right\} \mapsto\{1, \ldots, N\}$, and where $\mathcal{A}\left(\{q\},\{p\},\{\nu\}, f_{\vec{\nu}}\right)$ is a bounded function of $p_{j}$ if $\nu_{j}=0$, and independent of $p_{j}$ otherwise.

There are however some noteworthy differences from section 3.1 brought by the presence of factors involving the function $f(p)$ and the fact that there is still a $p_{i}$ dependence in the sum (82) outside the partial fraction decomposition. In place of (43) we now have

$$
\begin{gathered}
\frac{4}{L} \sum_{p>0, \in \mathrm{R}} \frac{\sin p \sin q^{\prime} f(p) / f\left(q^{\prime}\right)}{\cos q-\cos p} e^{2 i t \varepsilon(p)}=2 i \operatorname{sgn}\left(t \varepsilon^{\prime}(q)\right) \sin q^{\prime} \frac{f(q)}{f\left(q^{\prime}\right)} e^{2 i t \varepsilon(q)}+\mathcal{O}\left(L^{0} t^{-1 / 2}\right) \\
\frac{4}{L^{2}} \sum_{p>0, \in \mathrm{R}} \frac{\sin p \sin q f(p) / f(q)}{(\cos q-\cos p)^{2}} e^{2 i t \varepsilon(p)}=\left(1-\frac{4 t\left|\varepsilon^{\prime}(q)\right|}{L}+\frac{2 i \operatorname{sgn}\left(\varepsilon^{\prime}(q)\right)}{L} \frac{f^{\prime}(q)}{f(q)}\right) e^{2 i t \varepsilon(q)} \\
+\mathcal{O}\left(L^{-1} t^{-1 / 2}\right),
\end{gathered}
$$

as shown in appendix A. The analog of eqn 47 is given by

$$
\begin{aligned}
S_{n, 2 m}=\frac{(2 i)^{n} S_{0,0}}{(-2)^{m} L^{n+2 m}} \sum_{\substack{q_{1}^{0}<\ldots<q_{m}^{0} \\
q_{1}^{1}<\ldots<q_{n}^{1} \\
q_{1}^{2}<\ldots<q_{m}^{2} \\
\text { all distinct }}} A\left(\left\{q^{0}\right\},\left\{q^{1}\right\},\left\{q^{2}\right\}\right) \prod_{j=1}^{m}\left(4 \sin q_{j}^{0} \sin q_{j}^{2} \frac{f\left(q_{j}^{2}\right)}{f\left(q_{j}^{0}\right)}\right) \\
\qquad e^{2 i t \sum_{i=1}^{m} \varepsilon\left(q_{i}^{2}\right)-\varepsilon\left(q_{i}^{0}\right)} \prod_{i=1}^{n} \sin q_{i}^{1} \operatorname{sgn}\left(\varepsilon^{\prime}\left(q_{i}^{1}\right)\right),
\end{aligned}
$$

with

$$
S_{0,0}=\sqrt{\xi} \exp \left(-4 \int_{0}^{\pi}\left|t \varepsilon^{\prime}(x)\right| \rho(x) d x\right) .
$$

Here we used that $f(p)(83)$ fulfils

$$
\int_{0}^{\pi} \frac{f^{\prime}(x)}{f(x)} \rho(x) d x=0 .
$$

The possibility of differentiating $f(p)$ also modifies the derivation of (61), which now takes the form

$$
\begin{aligned}
& A\left(\left\{q_{i}\right\}_{i \in I_{1} \cup I_{0}},\{\tilde{\nu}\}, \tilde{f}\right)= \\
& \sum_{K \subset I_{1}} \prod_{k \in K} \frac{f^{\prime}\left(q_{k}\right)}{f\left(q_{k}\right)} \sum_{P \text { pairings of } I_{1}-K} \prod_{(i, j) \in P}\left(\frac{1}{\left(\cos q_{i}-\cos q_{j}\right)^{2}}+4 \frac{\partial_{\cos k} \varepsilon\left(q_{i}\right) \partial_{\cos k} \varepsilon\left(q_{j}\right)}{\left(\varepsilon\left(q_{i}\right)+\varepsilon\left(q_{j}\right)\right)^{2}}\right) .
\end{aligned}
$$

Because of 899, however, only $K=\{\}$ remains after the sum over $q_{k}$.

\subsubsection{Result: $\mathcal{O}\left(\rho_{Q}^{2}\right)$ uniformly in $t$ at large $t$}

The final result of the above calculation for the time evolution of the order parameter one-point function after a quench of the transverse field within the ordered phase is

$$
\left\langle\sigma_{\ell}^{x}(t)\right\rangle=C \exp \left(-4 \int_{0}^{\pi} \rho(x)(1+2 \pi \rho(x))\left|t \varepsilon^{\prime}(x)\right| d x\right),
$$


where

$$
C=\sqrt{\xi} \exp \left(-4 \int_{0}^{\pi} d x \int_{0}^{\pi} d y \rho(y)\left[\frac{\rho^{\prime}(x) \sin y}{\cos y-\cos x}-2 \frac{\left|\varepsilon^{\prime}(x) \varepsilon^{\prime}(y)\right|}{(\varepsilon(x)+\varepsilon(y))^{2}} \rho(x)\right]\right) .
$$

This result holds at the second order in the density $\mathcal{O}\left(\rho_{Q}^{2}\right)$. The reader may have noted that, since $\varepsilon^{\prime}(0)=\varepsilon^{\prime}(\pi)=0$, the quantum quench dynamics is a 'time-like region' case, so the saddle point effects might modify this prefactor at order $\rho^{1}$. It turns out, however, that $\rho(0)=\rho^{\prime}(0)=\rho(\pi)=\rho^{\prime}(\pi)=0$, so the saddle point corrections are higher order in time and do not affect the prefactor.

\subsection{Comments on the form factor summation}

Having carried out form factor summations to obtain the leading late-time asymptotics in the low-density regime in both the finite temperature and the quantum quench contexts it is useful to take stock and stress some features that we expect to be of a general nature.

\subsubsection{Which states govern the late time dynamics?}

The leading late time behaviour follows from eqns (43) that enter the sum over $p$ 's of the partial fraction decomposition (39). These formulas are obtained by isolating the singularity and dropping the integral of an oscillatory bounded function, $c f$. Appendix A. The singular part involves momenta $p_{j}$ in (33) that are at distance $\mathcal{O}\left(L^{-1}\right)$ of any of the $q_{i}$. The aim of this section is to quantify more precisely the number of $p_{j}$ that have to be summed in order to recover the late time dynamics. In other words, we would like to know the smallest function $\eta(L)$ such that

$$
\sum_{n=-L \eta(L)}^{L \eta(L)} \frac{e^{i t\left(\bar{\varepsilon}(q)-\bar{\varepsilon}\left(p_{n}\right)\right)}}{L^{2} \sin ^{2}\left(\frac{p_{n}-q}{2}\right)}=\left(1-\frac{2\left|t \bar{\varepsilon}^{\prime}(q)\right|}{L}\right),
$$

with $p_{n}=q+\frac{2 \pi}{L}(n+1 / 2)$. We first observe that if $L \eta(L) \rightarrow N_{0}$ remains finite when we take $L \rightarrow \infty$, then

$$
\begin{aligned}
\sum_{n=-L \eta(L)}^{L \eta(L)} \frac{e^{i t\left(\bar{\varepsilon}(q)-\bar{\varepsilon}\left(p_{n}\right)\right)}}{L^{2} \sin ^{2}\left(\frac{p_{n}-q}{2}\right)} & \approx \sum_{n=-L \eta(L)}^{L \eta(L)} \frac{1}{L^{2} \sin ^{2}\left(\frac{p_{n}-q}{2}\right)}+\mathcal{O}\left(t^{2} / L^{2}\right) \\
& \approx \sum_{n=-N_{0}}^{N_{0}} \frac{1}{\pi^{2}(n+1 / 2)^{2}}<\sum_{n=-\infty}^{\infty} \frac{1}{\pi^{2}(n+1 / 2)^{2}}=1 .
\end{aligned}
$$

As our spectral representation involves $N \propto L$ sums of this kind we obtain an infinite product over factors that are strictly smaller than 1 and obtain a vanishing answer. Hence retaining only a finite number of $p_{j}$ 's in our sum is clearly insufficient.

Conversely, if $\eta(L)=\eta$ stays finite we do have (93) at leading order in time because the terms we drop compared to having limits $\pm \infty$ contribute to an oscillatory integral of a bounded function

$$
\int_{\eta}^{\infty} \frac{e^{i t x}}{x^{2}} d x+\int_{-\infty}^{-\eta} \frac{e^{i t x}}{x^{2}} d x
$$

that vanishes at large times.

What if now $\eta(L) \rightarrow 0$ with $L \eta(L) \rightarrow \infty$ ? We have, by turning the sum into an integral with Euler-Maclaurin correction terms

$$
\sum_{n>\eta(L) L} \frac{e^{i \frac{\theta}{L}(n+1 / 2)}}{(n+1 / 2)^{2}}=\frac{1}{L} \int_{\eta}^{\infty} \frac{e^{i \theta x}}{x^{2}} d x+\mathcal{O}\left(\frac{1}{L^{n} \eta^{n}}, n \geq 3\right),
$$


and

$$
\int_{\eta}^{\infty} \frac{e^{i \theta x}}{x^{2}} d x=\frac{1}{\eta} E_{2}(-i \theta \eta)=\frac{i}{\eta^{2} \theta} e^{i \eta \theta}+\mathcal{O}\left(\frac{1}{\theta^{n+2} \eta^{n+3}}, n \geq 0\right),
$$

obtained from the expansion of the exponential integral $E_{2}(x)=\int_{1}^{\infty} \frac{e^{-x u}}{u^{2}} d u$. If we want this term to become negligible compared to (43) at late times in the scaling limit, then we need $L \eta^{n+2}(L) \rightarrow \infty$ for all $n$ in order to ensure that both the Euler MacLaurin correction terms in (96) and (97) are negligible. Hence any power-law $\eta(L)=L^{-\nu}$ with $\nu>0$ will not suffice. Stated differently, the number $L \eta(L)$ has to be larger than any $L^{\nu}$ for $0<\nu<1$, but any macroscopic fraction $\epsilon L$ with $\epsilon>0$ is sufficient. We will denote by mesoscopic this number of states (in contrast with microscopic $\mathcal{O}(1)$ and macroscopic $\mathcal{O}(L)$ ).

Finally, it is clear from e.g. (48) that in order to recover an exponential decay in time, one should multiply a $\mathcal{O}(L)$ number of terms like 433 , and to recover the right exponent one should take into account 'almost all' these terms at each momentum $q$.

We conclude that if the sum over the $p_{j}$ in $(33)$ is viewed in terms of particle-hole excitations over the $q_{i}$, the leading late time behaviour emerges from a mesoscopic number (i.e. larger than any $L^{\nu}$ for $0<\nu<1$, but smaller than $\epsilon L$ for any $\epsilon>0$ ) of particlehole excitations around each $q_{i}$. It represents an exponential number of states, but still sub-entropic, in the sense that it includes only states whose macroscopic state is the representative state itself. We expect this to be a general feature of form factor expansions of semi-local operators that holds also in interacting theories.

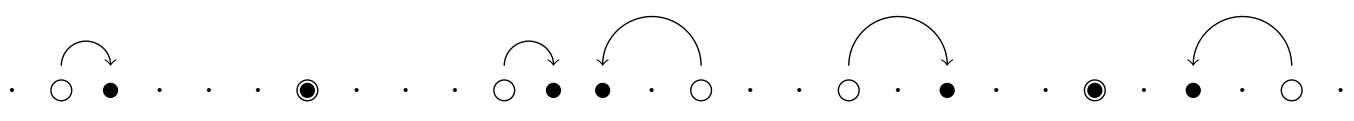

Figure 9: Sketch of the states contributing to the leading asymptotics: position of the momenta of the representative state (empty circles), position of the momenta of the intermediate state (filled circles), and position of the holes (dots).

\subsubsection{Non-vanishing low-density limit of form factors}

The leading order term both in time and density (48), obtained by keeping only the double poles in the partial fraction decomposition (39), has an interesting physical interpretation in terms of low-density limit of the form factor $(19)$. This limit consists in assuming that $\rho(x)$ is small everywhere, i.e. that $L\left(q_{i+1}-q_{i}\right) \rightarrow \infty$ when $L \rightarrow \infty$ in the scaling limit ${ }^{3}$. One can observe then that the square of the form factor (37) vanishes in the scaling limit $L \rightarrow \infty$ unless each $p$ is at a distance of order $\mathcal{O}\left(L^{-1}\right)$ from a $q$, and because of the low-density assumption this $q$ becomes unique in the limit $L \rightarrow \infty$. Thus one can write

$$
p_{i}=q_{i}+\frac{2 \pi}{L}\left(n_{i}+1 / 2\right),
$$

with $n_{i}$ an integer. In the low-density limit we have

$$
\lim _{L \rightarrow \infty} \frac{\sin \frac{q_{j}-q_{j^{\prime}}}{2} \sin \frac{p_{j}-p_{j^{\prime}}}{2}}{\sin \frac{q_{j}-p_{j^{\prime}}}{2} \sin \frac{q_{j^{\prime}}-p_{j}}{2}}=-1, \quad \lim _{L \rightarrow \infty} \frac{\varepsilon_{q_{j} p_{j^{\prime}}} \varepsilon_{q_{j^{\prime}} p_{j}}}{\varepsilon_{q_{j} q_{j^{\prime}}} \varepsilon_{p_{j} p_{j^{\prime}}}}=1, \quad \text { for } j<j^{\prime},
$$

\footnotetext{
${ }^{3}$ Strictly speaking, this is a different limit than merely $N / L \ll 1$, since this latter one does not require $\rho(x)=\mathcal{O}(N / L)$ everywhere. For example, a ground state at zero temperature and high chemical potential would have $N / L \ll 1$ but not $\rho(0) \ll 1$.
} 
and so in the scaling limit

$$
\left|\left\langle q_{1}, \ldots, q_{N}\left|\sigma_{l}^{x}\right| p_{1}, \ldots, p_{N}\right\rangle\right|^{2}=\frac{\xi}{\pi^{2 N}} \prod_{j=1}^{N} \frac{1}{\left(n_{j}+1 / 2\right)^{2}},
$$

which constitutes the low-density approximation of the form factor in the case it is not vanishing in the scaling limit.

Now, we trade the $1 / N$ ! in (33) for an ordering $p_{1}<\ldots<p_{N}$ and choose an ordering $q_{1}<\ldots<q_{N}$. Such a constraint translates into a constraint on the $n_{j}$ in (98), namely $n_{j+1}>n_{j}-\frac{L}{2 \pi}\left(q_{j+1}-q_{j}\right) \approx n_{j}-\frac{1}{2 \pi \rho\left(q_{j}\right)}$. At leading order in $\rho$, this constraint becomes $-\frac{1}{4 \pi \rho\left(q_{j}\right)}<n_{j}<\frac{1}{4 \pi \rho\left(q_{j}\right)}$, so that $(33)$ becomes

$$
\chi^{x x}(\ell, t)=\sum_{n_{1}=-\left\lceil\frac{1}{4 \pi \rho\left(q_{1}\right)}\right\rceil}^{\left\lfloor\frac{1}{4 \pi \rho\left(q_{1}\right)}\right\rfloor} \sum_{n_{N}=\left\lceil-\frac{1}{4 \pi \rho\left(q_{N}\right)}\right\rceil}^{\left\lfloor\frac{1}{4 \pi \rho\left(q_{N}\right)}\right\rfloor} \frac{\xi}{\pi^{2 N}} \prod_{j=1}^{N} \frac{e^{-2 i \pi t \sum_{j=1}^{N} \bar{\varepsilon}^{\prime}\left(q_{j}\right) \frac{\left(n_{j}+1 / 2\right)}{L}}}{\left(n_{j}+1 / 2\right)^{2}} .
$$

Hence in the low density limit $\rho(q) \rightarrow 0$ we have

$$
\chi^{x x}(\ell, t)=\sum_{n_{1}=-\infty}^{\infty} \ldots \sum_{n_{N}=-\infty}^{\infty} \frac{\xi}{\pi^{2 N}} \prod_{j=1}^{N} \frac{e^{-2 i \pi t \sum_{j=1}^{N} \bar{\varepsilon}^{\prime}\left(q_{j}\right) \frac{\left(n_{j}+1 / 2\right)}{L}}}{\left(n_{j}+1 / 2\right)^{2}} .
$$

This expression factorizes and can be computed along (48) with formulas (158).

\subsubsection{Static correlations}

Results (66), (78), (79) and their analogues hereafter (140), (147) for the finite temperature correlations are obtained in the regime $\ell, t \rightarrow \infty$ at fixed $\alpha=t / \ell$. In their derivation, however, we have only used that the phase $i t(E(\{q\})-E(\{p\}))+i \ell(P(\{p\})-P(\{q\}))$ in (33) is large, therefore our calculations are also applicable to static correlations $t=0$ at large $\ell$. It amounts to replacing $t \bar{\varepsilon}(x)$ by $-\ell x$ in all the phases $e^{-i t \bar{\varepsilon}(x)}$. All the oscillatory integrals that we neglected because of their large time behaviour $\sim t^{-1 / 2}$ will now decay at large distances at least as $\ell^{-1}$ (and in general much faster). In particular (43) still hold, but with corrections $\mathcal{O}\left(L^{0} \ell^{-1}\right)$ and $\mathcal{O}\left(L^{-1} \ell^{-1}\right)$ respectively. Static correlations are then obtained by replacing $\left|t \bar{\varepsilon}^{\prime}(x)\right|$ (i.e., $\left.\left|t \varepsilon^{\prime}(x)-\ell\right|\right)$ by $|\ell|$ in the final results.

\section{Quantum quench dynamics beyond low densities}

The framework presented in section 3 is general and permits us to compute the late time behaviour and subleading corrections of form factor sums, order by order in $\rho_{Q}=N / L$. In particular, it yields the expression of the observables of interest as $C e^{-t / \tau}$, where both $C$ and $\tau$ are the exact expressions at a given order (here second order) in $\rho_{Q}$. The computation of a generic order in the density is however rather involved. In this section and in the following one we focus on the exponent $\tau$ of the exponential decay, for which another more efficient but less general approach can be used to calculate it at all orders in $\rho_{Q}$, i.e. writing the correlation function as $e^{-t / \tau}$ where $\tau$ includes all orders in $\rho_{Q}$.

This first section treats the quantum quench problem introduced in subsection 2.1 . 


\subsection{Determinant representation}

As shown in section 3.2, the leading contribution in 27 is obtained for $N=M$, on which we will focus. The starting observation is that the last term of 81 can be written as a Cauchy determinant, indeed we have

$$
\frac{\prod_{i \neq j}\left(\cos q_{i}-\cos q_{j}\right)\left(\cos p_{i}-\cos p_{j}\right)}{\prod_{i, j}\left(\cos q_{i}-\cos p_{j}\right)^{2}}=(\operatorname{det} C)^{2}=\operatorname{det} C^{T} C, \quad C_{i j}=\frac{1}{\cos p_{i}-\cos q_{j}} .
$$

Let us define $\bar{M}=C^{T} C$ and $M_{i k}^{j}=C_{i j} C_{k j}$, so that $\bar{M}_{i k}=\sum_{j} M_{i k}^{j}$. The determinant of $\bar{M}$ can be expanded as follows

$$
\begin{aligned}
\operatorname{det} \bar{M} & =\sum_{\tau \in \mathfrak{S}_{N}} \operatorname{sgn}(\tau) \bar{M}_{1 \tau(1)} \ldots \bar{M}_{N \tau(N)} \\
& =\sum_{j_{1}, \ldots, j_{N} \in\{1, \ldots, N\}} \sum_{\tau \in \mathfrak{S}_{N}} \operatorname{sgn}(\tau) M_{1 \tau(1)}^{j_{1}} \ldots M_{N \tau(N)}^{j_{N}} .
\end{aligned}
$$

The term $M_{a \tau(a)}^{j_{a}} M_{b \tau(b)}^{j_{b}}$ is invariant under the replacement $\tau \rightarrow \tau \cdot(a b)$ if $j_{a}=j_{b}$, whereas the sign of the permutation changes. Hence the sum over $\tau$ vanishes unless all the $j$ 's are distinct, i.e., if they are a permutation of $1, \ldots, N$. In conclusion we find

$$
\operatorname{det} \bar{M}=\sum_{\sigma \in \mathfrak{S}_{N}} \operatorname{det} M^{\sigma}
$$

with $M_{i k}^{\sigma}=M_{i k}^{\sigma(i)}$. This relation permits one to eliminate the $1 / N$ ! factor in 82 , which then reads

$$
\left\langle\sigma_{\ell}^{x}(t)\right\rangle=\operatorname{Re}\left[\frac{\sqrt{\xi}}{L^{2 N}} \sum_{0<p_{1}, \ldots, p_{N}} \prod_{j=1}^{N} 4 \frac{f\left(p_{j}\right)}{f\left(q_{j}\right)} \sin p_{j} \sin q_{j} e^{2 i t\left(\varepsilon\left(p_{j}\right)-\varepsilon\left(q_{j}\right)\right)} \prod_{i, j} \frac{\varepsilon_{q_{j} p_{i}}^{4}}{\varepsilon_{q_{j} q_{i}}^{2} \varepsilon_{p_{j} p_{i}}^{2}} \operatorname{det} M\right]
$$

where $f(p)$ is defined in $(83)$ and $M$ is explicitly given by

$$
M_{i j}=\frac{1}{\cos q_{i}-\cos p_{i}} \frac{1}{\cos q_{j}-\cos p_{i}} .
$$

\subsubsection{Approximation}

In order to proceed we now drop one of the factors in (106), which we argue is justified at late times. The factor involving the $\varepsilon_{k, k^{\prime}}$ in 106 is a function

$$
g_{\left\{q_{1}, \ldots, q_{N}\right\}}\left(p_{1}, \ldots, p_{N}\right)=\prod_{i, j} \frac{\varepsilon_{q_{j} p_{i}}^{4}}{\varepsilon_{q_{j} q_{i}}^{2} \varepsilon_{p_{j} p_{i}}^{2}}
$$

such that for $\sigma \in \mathfrak{S}_{N}$

(i) $g_{\left\{q_{1}, \ldots, q_{N}\right\}}\left(p_{1}, \ldots, p_{N}\right)$ is regular, symmetric and has no poles in $p_{1}, \ldots, p_{N}$;

(ii) $g_{\left\{q_{1}, \ldots, q_{N}\right\}}\left(q_{\sigma(1)}, \ldots, q_{\sigma(N)}\right)=1$;

(iii) $\forall k=1, \ldots, N,\left.\quad g_{\left\{q_{1}, \ldots, q_{N}\right\}}\left(p_{1}, \ldots, p_{N}\right)\right|_{\forall j=k+1, \ldots, N, p_{j}=q_{\sigma(j)}}=g_{\left\{q_{\sigma(1)}, \ldots, q_{\sigma(k)}\right\}}\left(p_{1}, \ldots, p_{k}\right)$;

(iv) $\forall i=1, \ldots, N,\left.\quad \partial_{p_{i}} g_{\left\{q_{1}, \ldots, q_{N}\right\}}\left(p_{1}, \ldots, p_{N}\right)\right|_{\forall j p_{j}=q_{\sigma(j)}}=0$.

The first two properties (i) and (ii) follow immediately from the definition (20). The third one (iii) means that if some $p$ 's are set to some $q$ 's in a one-to-one fashion, then one 
recovers the same function $g$ with the remaining $p$ 's and $q$ 's. As for property (iv), it means that (ii) holds at order $\left(p_{i}-q_{\sigma(i)}\right)^{2}$.

We will now argue that by virtue of these properties setting $g$ to 1 does not affect the leading behaviour at late times $t$. First, property (i) ensures that $g$ does not modify the general structure (39) by allowing e.g. for higher order poles, or poles at other momenta. Property (ii) ensures that $g$ does not modify the values $A\left(I_{0}, I_{1}, I_{2}\right)$ when $I_{1}=\{\}$, because in these cases there is always a double zero to differentiate and $g$ is then evaluated at a permutation of the momenta. When $I_{1} \neq\{\}$, the function $g$ does change $A\left(I_{0}, I_{1}, I_{2}\right)$, but, because of property (iv), it will not modify the pairing structure of (61), and will only modify the factors in (61) by an extra additive term. Finally, property (iii) allows one to repeat these steps recursively in (56). We now observe that the resulting partial fraction decomposition will always boil down to evaluating sums of the form (173) and (181). These are divergent as $L \rightarrow \infty$, cf. (175) and (184), and hence can only appear together, which cancels the divergence. Sums of type (173) emerge from $I_{2}$, which is not affected by $g$, and the ones of type (181) come from $I_{0}$, which is instead affected by $g$. Since the $t$ dependence is carried by $(173)$, the leading time behaviour will never depend on $g$.

Based on these arguments we now make the approximation of setting $g=1$

$$
\begin{aligned}
\left\langle\sigma_{\ell}^{x}(t)\right\rangle & \approx \operatorname{Re} \frac{4^{N} \sqrt{\xi}}{L^{2 N}} \sum_{\substack{0 p_{1}, \ldots, p_{N} \\
\in \mathrm{R}}} \operatorname{det}_{i, j}\left|\frac{e^{i t\left(2 \varepsilon\left(p_{i}\right)-\varepsilon\left(q_{i}\right)-\varepsilon\left(q_{j}\right)\right)} \sin p_{i} \sin q_{j} f\left(p_{i}\right) / f\left(q_{j}\right)}{\left(\cos q_{i}-\cos p_{i}\right)\left(\cos q_{j}-\cos p_{i}\right)}\right| \\
& =\sqrt{\xi} \operatorname{det}(A)
\end{aligned}
$$

where the matrix $A$ is given by

$$
A_{i j}=\frac{4}{L^{2}} \sum_{0<p \in \mathrm{R}} \frac{e^{i t\left(2 \varepsilon(p)-\varepsilon\left(q_{i}\right)-\varepsilon\left(q_{j}\right)\right)} \sin p \sin q_{j} f(p) / f\left(q_{j}\right)}{\left(\cos q_{i}-\cos p\right)\left(\cos q_{j}-\cos p\right)}, \quad i, j=1, \ldots, N
$$

\subsection{Asymptotic forms of the matrix elements}

In the next step we work out the large- $L$ asymptotics of the matrix elements $A_{i j}$.

\subsubsection{Diagonal matrix elements}

The diagonal matrix elements were already computed in (86), cf. Appendix A. They read

$$
A_{i i}=1-\frac{\left|\theta_{i}\right|}{\pi}+\frac{2 i \operatorname{sgn}\left(t \varepsilon^{\prime}\left(q_{i}\right)\right)}{L} \frac{f^{\prime}\left(q_{i}\right)}{f\left(q_{i}\right)}+\mathcal{O}\left(L^{-1} t^{-1 / 2}\right),
$$

where we have defined

$$
\theta_{i}=\frac{4 \pi t \varepsilon^{\prime}\left(q_{i}\right)}{L}
$$

\subsubsection{Off-diagonal matrix elements}

To compute the off-diagonal elements, we write

$$
\frac{1}{\left(\cos q_{i}-\cos p\right)\left(\cos q_{j}-\cos p\right)}=\frac{1}{\cos q_{i}-\cos q_{j}}\left(\frac{1}{\cos q_{j}-\cos p}-\frac{1}{\cos q_{i}-\cos p}\right),
$$

and use the first equation in (86), see also appendix A. We obtain

$$
A_{i j}=\frac{2 i \operatorname{sgn}\left(t \varepsilon^{\prime}\left(q_{j}\right)\right) \sin q_{j}}{L\left(\cos q_{i}-\cos q_{j}\right)}\left[e^{i t\left(\varepsilon\left(q_{j}\right)-\varepsilon\left(q_{i}\right)\right)}-\frac{f\left(q_{i}\right) \operatorname{sgn}\left(\varepsilon^{\prime}\left(q_{j}\right) \varepsilon^{\prime}\left(q_{i}\right)\right)}{f\left(q_{j}\right)} e^{i t\left(\varepsilon\left(q_{i}\right)-\varepsilon\left(q_{j}\right)\right)}\right] .
$$




\subsubsection{Approximate determinant representation}

Combining (112) and 115) provides the following approximate determinant representation

$$
\left\langle\sigma_{\ell}^{x}(t)\right\rangle \approx \operatorname{Re} \sqrt{\xi} \operatorname{det}(I-\Xi),
$$

where

$$
\begin{aligned}
\Xi_{i j} & =\delta_{i j}\left[\frac{\left|\theta_{i}\right|}{\pi}-\frac{2 i \operatorname{sgn}\left(t \varepsilon^{\prime}\left(q_{i}\right)\right)}{L} \frac{f^{\prime}\left(q_{i}\right)}{f\left(q_{i}\right)}\right] \\
& +\left(1-\delta_{i j}\right) \frac{2 i \operatorname{sgn}\left(t \varepsilon^{\prime}\left(q_{j}\right)\right) \sin q_{j}}{L\left(\cos q_{j}-\cos q_{i}\right)}\left[e^{i t\left(\varepsilon\left(q_{j}\right)-\varepsilon\left(q_{i}\right)\right)}-\frac{f\left(q_{i}\right) \operatorname{sgn}\left(\varepsilon^{\prime}\left(q_{j}\right) \varepsilon^{\prime}\left(q_{i}\right)\right)}{f\left(q_{j}\right)} e^{i t\left(\varepsilon\left(q_{i}\right)-\varepsilon\left(q_{j}\right)\right)}\right] \\
& +\mathcal{O}\left(L^{-1} t^{-1 / 2}\right) .
\end{aligned}
$$

\subsection{Evaluating the determinant}

We now write $\operatorname{det} M=\exp \operatorname{tr} \log M$ and use the expansion

$$
\log (I-\Xi)=-\sum_{n \geq 1} \frac{\Xi^{n}}{n}
$$

\subsubsection{First order}

The first order gives

$$
\begin{aligned}
\operatorname{tr} \Xi & =4|t| \int_{0}^{\pi}\left|\varepsilon^{\prime}(x)\right| \rho(x) d x+2 i \int_{0}^{\pi} \frac{f^{\prime}(x)}{f(x)} \rho(x) d x+\mathcal{O}\left(L^{0} t^{-1 / 2}\right) \\
& =4|t| \int_{0}^{\pi}\left|\varepsilon^{\prime}(x)\right| \rho(x) d x+\mathcal{O}\left(L^{0} t^{-1 / 2}\right) .
\end{aligned}
$$

\subsubsection{Second order}

Since $\sum_{i} \Xi_{i i}^{2}=\mathcal{O}\left(L^{-1}\right)$ the second order reads

$$
\operatorname{tr}\left(\Xi^{2}\right)=\sum_{i \neq j} \Xi_{i j} \Xi_{j i}+\mathcal{O}\left(L^{-1}\right)=\Omega_{1}+\Omega_{2}+\mathcal{O}\left(L^{-1}\right)
$$

where

$$
\Omega_{1}=\frac{8}{L^{2}} \sum_{i \neq j} \frac{\sin q_{i} \sin q_{j}}{\left(\cos q_{i}-\cos q_{j}\right)^{2}}, \quad \Omega_{2}=-\frac{8}{L^{2}} \sum_{i \neq j} \frac{\sin q_{i} \sin q_{j} \frac{f\left(q_{i}\right)}{f\left(q_{j}\right)}}{\left(\cos q_{i}-\cos q_{j}\right)^{2}} e^{2 i t\left(\varepsilon\left(q_{i}\right)-\varepsilon\left(q_{j}\right)\right)} .
$$

These sums are computed in appendix A, We obtain

$$
\operatorname{tr}\left(\Xi^{2}\right)=4|t| \int_{0}^{\pi} d x\left|\varepsilon^{\prime}(x)\right| 4 \pi \rho(x)^{2}+8 \int d x d y \frac{\sin y}{\cos y-\cos x} \rho^{\prime}(x) \rho(y)+\mathcal{O}\left(t^{-1 / 2}\right) .
$$

Once exponentiated in the determinant, we recognize the terms obtained earlier with the partial fraction decomposition approach (91), (92), but without the contributions involving the $\varepsilon$ in the prefactor $C$. This difference is a direct consequence of our approximation $g=1$. 


\subsubsection{Leading late-time contribution at all orders}

In order to compute the $O(t)$ term at higher orders in $\rho$, we first notice that it can only arise from the second order poles in the matrix entries, hence by pairs of momenta separated by $o\left(L^{0}\right)$. In this regime the matrix elements become

$$
\Xi_{i j}=\delta_{i j} \frac{\left|\theta_{i}\right|}{\pi}+\left(1-\delta_{i j}\right) \frac{-i \operatorname{sgn}(\theta)}{\pi} \frac{e^{i \theta_{i} T_{i} \Delta_{i j} / 2}-e^{-i \theta_{i} T_{i} \Delta_{i j} / 2}}{T_{i} \Delta_{i j}}
$$

with $\Delta_{i j}=i-j$. We obtain in this regime

$$
\begin{aligned}
\operatorname{tr}\left(\Xi^{n}\right) & =\sum_{i_{1}, i_{2}, \ldots, i_{n}} \Xi_{i_{1} i_{2}} \ldots \Xi_{i_{n} i_{1}} \\
& =-\sum_{i} \frac{1}{\left(i \operatorname{sgn}\left(\theta_{i}\right) \pi\right)^{n} T_{i}^{n}} \sum_{\Delta_{1}, \ldots, \Delta_{n-1} \neq 0}\left[\prod_{m=1}^{n-1} \frac{1-e^{-i \theta_{i} T_{i} \Delta_{m}}}{\Delta_{m}}\right] \frac{1-e^{i \theta_{i} T_{i}\left(\Delta_{1}+\ldots+\Delta_{n-1}\right)}}{\Delta_{1}+\ldots+\Delta_{n-1}} .
\end{aligned}
$$

The sums over $\Delta_{j}$ can be carried out using that for $\Delta^{\prime} \neq 0$

$$
\sum_{\Delta \neq 0,-\Delta^{\prime}} \frac{1-e^{-i \theta \Delta}}{\Delta} \frac{1-e^{i \theta\left(\Delta+\Delta^{\prime}\right)}}{\Delta+\Delta^{\prime}}=2 i(\pi-|\theta|) \operatorname{sgn}(\theta) \frac{1-e^{i \theta \Delta^{\prime}}}{\Delta^{\prime}},
$$

which is obtained from $\frac{1}{\Delta\left(\Delta+\Delta^{\prime}\right)}=\frac{1}{\Delta^{\prime}}\left(\frac{1}{\Delta}-\frac{1}{\Delta+\Delta^{\prime}}\right)$ and relations 158 by carefully treating the cases $\Delta=0,-\Delta^{\prime}$. Using that $\theta=\mathcal{O}\left(L^{-1}\right)$ to neglect the $|\theta|$ term in 125 we arrive at

$$
\begin{aligned}
\operatorname{tr}\left(\Xi^{n}\right) & =-\sum_{i} \frac{\left(2 i \operatorname{sgn}\left(\theta_{i}\right) \pi\right)^{n-2}}{\left(i \operatorname{sgn}\left(\theta_{i}\right) \pi\right)^{n} T_{i}^{n}} \sum_{\Delta_{1} \neq 0} \frac{\left(1-e^{-i \theta_{i} T_{i} \Delta_{1}}\right)\left(1-e^{i \theta_{i} T_{i} \Delta_{1}}\right)}{\Delta_{1}^{2}}+\mathcal{O}\left(L^{-1}\right) \\
& =\sum_{i} \frac{2^{n-1}}{\pi^{2} T_{i}^{n}}\left(2 \operatorname{Li}_{2}(1)-\operatorname{Li}_{2}\left(e^{i \theta_{i} T_{i}}\right)-\operatorname{Li}_{2}\left(e^{-i \theta_{i} T_{i}}\right)\right)+\mathcal{O}\left(L^{-1}\right) \\
& =\sum_{i} \frac{2^{n-1}\left|\theta_{i}\right|}{\pi T_{i}^{n-1}}+\mathcal{O}\left(L^{-1}\right)=4|t| \int_{0}^{\pi}\left|\varepsilon^{\prime}(x)\right|(4 \pi \rho(x))^{n-1} \rho(x) d x+\mathcal{O}\left(L^{-1}\right) .
\end{aligned}
$$

\subsubsection{Influence of the boundaries}

In the discussion above the momenta $p$ are constrained to be positive. In order to use eqns (43) we therefore had to neglect possible boundary effects for $q$ 's close to zero. We now verify that this does not influence the result. We have

$$
\sum_{n=-\eta_{1} L}^{\eta_{2} L} \frac{e^{i(n+1 / 2) \frac{w}{L} t}}{(n+1 / 2)^{2}}=\sum_{n=-\infty}^{\infty} \frac{e^{i(n+1 / 2) \frac{w}{L} t}}{(n+1 / 2)^{2}}-\frac{1}{\eta_{2} L} E_{2}\left(-i w \eta_{2} t\right)-\frac{1}{\eta_{1} L} E_{2}\left(i w \eta_{1} t\right)
$$

with $E_{2}(x)=\int_{1}^{\infty} \frac{e^{-x t}}{t^{2}} d t$ the exponential integral function. $L \eta_{1,2}$ are the number of vacancies between $0, \pi$ and $q_{i}$. They are $\eta_{1}=\frac{q_{i}}{2 \pi}$ and $\eta_{2}=\frac{\pi-q_{i}}{2 \pi}$. Hence the correction to $\operatorname{tr} \Xi$ is

$$
-\frac{2}{\pi} \int_{0}^{\pi}\left(\frac{E_{2}\left(-2 i \varepsilon^{\prime}(x) x t\right)}{x}+\frac{E_{2}\left(2 i \varepsilon^{\prime}(x)(\pi-x) t\right)}{\pi-x}\right) \rho(x) d x
$$

which goes to zero for large $t$ because the density vanishes quadratically at 0 and $\pi$. 


\subsection{Result: late-time asymptotics of the order parameter after a quench}

Substituting (119), 122 and (126) into 118 we arrive at the following result for the late-time asymptotics of the order parameter one-point function after a quench within the ferromagnetic phase

$$
\frac{\left\langle\Psi_{N}(t)\left|\sigma_{\ell}^{x}\right| \Psi_{s}(t)\right\rangle}{\left\langle\Psi_{N}(t) \mid \Psi_{s}(t)\right\rangle}=C \exp \left(\frac{|t|}{\pi} \int_{0}^{\pi}\left|\varepsilon^{\prime}(x)\right| \log (1-4 \pi \rho(x)) d x\right) \equiv C e^{-t / \tau}
$$

with $C$ given in 92 at order $\mathcal{O}\left(\rho_{Q}^{2}\right)$. The decay rate reproduces the exact result obtained in 50,51. However, the prefactor $C$ differs from the one conjectured in 50,51. We address this difference in subsection 4.5 below.

\subsection{Numerical Checks}

We now present some numerical checks of eqn 129 for the time evolution of the order parameter. In the limit of large separations $\ell \gg 2 J t$, using the Lieb-Robinson bound and the clustering properties of the initial state $|\Psi\rangle$, the two-point function factorizes into the product of two one-point functions that are identical by translational invariance

$$
\left\langle\Psi\left|\sigma_{\ell+1}^{x}(t) \sigma_{1}^{x}(t)\right| \Psi\right\rangle=\left\langle\Psi\left|\sigma_{1}^{x}(t)\right| \Psi\right\rangle^{2}+\mathcal{O}\left(e^{-\gamma(\ell-2 J t)}\right),
$$

with $\gamma$ a constant of order 1 . We can then obtain the one-point function $\left\langle\Psi\left|\sigma_{1}^{x}(t)\right| \Psi\right\rangle$ as the square root of the two-point function $\left\langle\sigma_{\ell+1}^{x}(t) \sigma_{1}^{x}(t)\right\rangle$ in the limit $\ell \gg 2 J t$, which can be efficiently computed numerically, as it can be expressed as the determinant of a block Toeplitz matrix even in the thermodynamic limit [51].

Numerical checks of the exponential decay in $(129)$ have already been reported in [51. Since our prediction (92) differs from the one conjectured in [51, we will focus on the prefactor $\mathcal{C}_{\mathrm{FF}}^{x}(\alpha)$ of the asymptotic behaviour of the two-point function in the limit $\ell, t \rightarrow \infty, \alpha=t / \ell$ fixed

$$
\begin{aligned}
\left\langle\Psi\left|\sigma_{\ell+1}^{x}(t) \sigma_{1}^{x}(t)\right| \Psi\right\rangle & \simeq \mathcal{C}_{\mathrm{FF}}^{x}(\alpha) \exp \left[\ell \int_{0}^{\pi} \frac{\mathrm{d} k}{\pi} \ln \left|\cos \Delta_{k}\right| \theta_{H}\left(2 \varepsilon_{h}^{\prime}(k) t-\ell\right)\right] \\
& \times \exp \left[2 t \int_{0}^{\pi} \frac{\mathrm{d} k}{\pi} \varepsilon_{h}^{\prime}(k) \ln \left|\cos \Delta_{k}\right| \theta_{H}\left(\ell-2 \varepsilon_{h}^{\prime}(k) t\right)\right]
\end{aligned}
$$

In [51] it was assumed that the constant $C_{\mathrm{FF}}^{x}(\alpha)$ is independent of $\alpha$. Calculating the asymptotics of the correlator for $\alpha \rightarrow \infty$ then leads to [52]

$$
C_{\mathrm{FF}}^{x}(\infty)=\frac{1-h h_{0}+\sqrt{\left(1-h^{2}\right)\left(1-h_{0}^{2}\right)}}{2 \sqrt{1-h h_{0}} \sqrt[4]{1-h_{0}^{2}}} .
$$

From $(92)$ it however follows $C \neq \sqrt{C_{\mathrm{FF}}^{x}(\infty)}$, suggesting in turn that $C_{\mathrm{FF}}^{x}(\alpha)$ is in fact $\alpha$ dependent. This is indeed supported by our numerical results, even though the difference $\left|C-\sqrt{C_{\mathrm{FF}}^{x}(\infty)}\right|$ is tiny. In Figs 10 we show that our results 129$)$ and $(92)$ are in agreement with numerical calculations of the order parameter one-point function. 

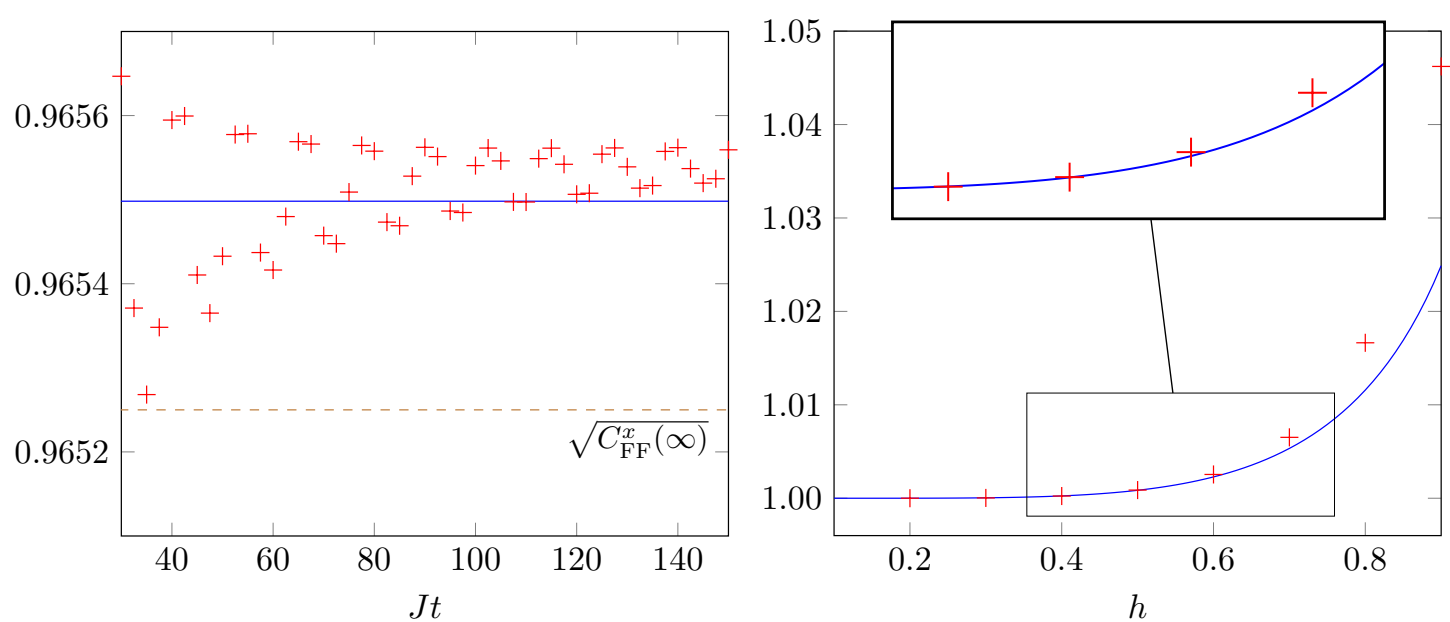

Figure 10: Left: Numerical results for $\left\langle\sigma_{1}^{x}(t)\right\rangle e^{t / \tau}$ with $\tau$ defined in (129) as a function of $t$ for a quench from $h_{0}=0.1$ to $h=0.5$, corresponding to a density $\rho_{Q}=0.0218$. The prefactor at order $\mathcal{O}\left(\rho_{Q}^{2}\right) 92$ is shown in blue and is seen to be compatible with our numerical results. For comparison we also show $\sqrt{C_{\mathrm{FF}}^{x}(\infty)}=0.96525$. Right: Numerically determined (red) and calculated leading order (blue) prefactor 92 divided by $\sqrt{\xi}$, for quenches from $h_{0}=0.1$ to $h$ as a function of $h$. Because of the oscillations, the measured value is an average of six points between times $t=75$ and 100 .

\section{Dynamical correlation functions at arbitrary finite tem- peratures}

In this section we follow the same reasoning as in section 4 but for dynamical correlation functions at finite temperature. We again treat the two cases $h<1$ and $h>1$ separately.

\subsection{Ordered phase $h<1$}

In the ordered phase, the sum in (33) involves states with the same number of particles as in the quench case (27). However, it is not possible to express each term as a Cauchy determinant as in section 4. This can however be overcome for the leading late time behaviour, where one can work with an approximate version of the form factor 19.

We focus again on intermediate states with $M=N$ in (33), which were argued in section 5.2 to give the leading late time behaviour. As discussed in section 4.1.1, the dominant contribution to the correlation function arises from the sums $(173)$, and the term proportional to $t$ on the right-hand side of 1173 has its origin in the isolated singularity $\frac{1}{x^{2}}$ in $\frac{1}{\sin ^{2} x}$. Hence, replacing terms of the form $\frac{1}{\sin ^{2} x}$ by $\frac{1}{x^{2}}$ in the form factor (19) will not affect the leading behaviour at late times. It follows that

$$
\lim _{t \rightarrow \infty} \frac{\tilde{\chi}^{x x}(\ell, t)}{\chi^{x x}(\ell, t)}=1
$$


where we have defined

$$
\begin{aligned}
\tilde{\chi}^{x x}(\ell, t) & =\frac{1}{N !} \sum_{\substack{p_{1}, \ldots, p_{N} \\
\in \in \mathbb{R}}} \tilde{F}_{\left\{p_{1}, \ldots, p_{N}\right\}}^{\left\{q_{1}, \ldots, q_{N}\right\}} e^{i t(E(\{q\})-E(\{p\}))+i \ell(P(\{p\})-P(\{q\}))}, \\
\tilde{F}_{\left\{p_{1}, \ldots, p_{N}\right\}}^{\left\{q_{1}, \ldots, q_{N}\right\}} & =\frac{\xi}{L^{2 N}} \frac{\prod_{i \neq j} \frac{q_{i}-q_{j}}{2} \frac{p_{i}-p_{j}}{2}}{\prod_{i, j}\left(\frac{q_{i}-p_{j}}{2}\right)^{2}}
\end{aligned}
$$

The form factor $\tilde{F}$ has the same Cauchy determinant structure we encountered in the quench case (103). Hence we can follow through the same steps and obtain

$$
\tilde{\chi}^{x x}(\ell, t)=\xi \operatorname{det}_{i, j=1, \ldots, N}\left[\frac{4}{L^{2}} \sum_{p \in \mathrm{R}} \frac{\left.e^{i \frac{t}{2}\left(\bar{\varepsilon}\left(q_{i}\right)+\bar{\varepsilon}\left(q_{j}\right)-2 \bar{\varepsilon}(p)\right.}\right)}{\left(q_{j}-p\right)\left(q_{i}-p\right)}\right] .
$$

The leading time behaviour of the coefficients of this matrix can be computing as in section 4.2 with formulas (158). One obtains

$$
\tilde{\chi}^{x x}(\ell, t)=\xi \operatorname{det}(1-\Xi)
$$

with

$$
\begin{aligned}
\Xi_{i j} & =\delta_{i j} \frac{\left|2 t \bar{\varepsilon}^{\prime}\left(q_{i}\right)\right|}{L} \\
& +\left(1-\delta_{i j}\right) \frac{2 i \operatorname{sgn}\left(t \bar{\varepsilon}^{\prime}\left(q_{j}\right)\right)}{L\left(q_{j}-q_{i}\right)}\left[e^{i \frac{t}{2}\left(\bar{\varepsilon}\left(q_{i}\right)-\bar{\varepsilon}\left(q_{j}\right)\right)}-\operatorname{sgn}\left(\bar{\varepsilon}^{\prime}\left(q_{j}\right) \bar{\varepsilon}^{\prime}\left(q_{i}\right)\right) e^{i \frac{t}{2}\left(\bar{\varepsilon}\left(q_{j}\right)-\bar{\varepsilon}\left(q_{i}\right)\right)}\right] \\
& +\mathcal{O}\left(L^{-1} t^{-1 / 2}\right) .
\end{aligned}
$$

Since one is interested only in the late time dynamics, we can focus on terms $i, j$ such that $q_{i}-q_{j}=o\left(L^{0}\right)$ as in 123 . Then one obtains the same formula as in 123

$$
\Xi_{i j}=\delta_{i j} \frac{\left|\theta_{i}\right|}{\pi}+\left(1-\delta_{i j}\right) \frac{-i \operatorname{sgn}(\theta)}{\pi} \frac{e^{i \theta_{i} T_{i} \Delta_{i j} / 2}-e^{-i \theta_{i} T_{i} \Delta_{i j} / 2}}{T_{i} \Delta_{i j}}
$$

with $\Delta_{i j}=i-j, T_{i}=\frac{1}{2 \pi \rho\left(q_{i}\right)}$ and $\theta_{i}=\frac{2 \pi t \bar{\varepsilon}^{\prime}\left(q_{i}\right)}{L}$.

\subsubsection{Two-point dynamical correlation functions in the ordered phase}

Following through the same steps as in the quench case we arrive at

$$
\chi^{x x}(\ell, t) \approx \exp \left(\frac{1}{2 \pi} \int_{-\pi}^{\pi}\left|t \varepsilon^{\prime}(x)-\ell\right| \log (1-4 \pi \rho(x)) d x\right),
$$

where, using the results of section 3 .

$$
C= \begin{cases}\xi \exp \left(-2 \int_{-\pi}^{\pi} \int_{-\pi}^{\pi} \frac{\rho(y) \rho^{\prime}(x)}{\tan \left(\frac{x-y}{2}\right)} d x d y\right) & \text { in the space like-region at order } \rho_{\beta}^{2} \\ \xi & \text { in the time like-region at order } \rho_{\beta}^{0} .\end{cases}
$$

As far as we know the result 140 has not previously been obtained in the literature. 


\subsubsection{Numerical checks}

In order to check the accuracy of 140 at finite times we have carried out numerical simulations following Ref. [84], where the two finite temperature dynamical two-point function for a finite open chain is computed exactly as a Pfaffian of a known matrix. As long as the two points are sufficiently far from the boundaries, then they take almost the same values as in an infinite chain. In Fig. 11 we compare 140 to numerical results in the space-like region by considering the logarithm of the correlator

$$
\mathcal{L}(\ell, t)=\ln \left(\left\langle\sigma_{\ell+1}^{x}(t) \sigma_{1}^{x}(0)\right\rangle\right) .
$$

For simplicity we take the extreme case $\alpha=0$, which corresponds to setting $t=0$. We recall indeed that static correlations are also covered by our calculations, as explained in section 3.5.3. In the left panel we plot (140) as a function of distance $\ell$ and mark by red crosses numerical results obtained for a chain of $L=200$ sites. We see that the asymptotic result $(140)$ is in excellent agreement for $\alpha=0$. In the right panel we test the accuracy of the $\mathcal{O}\left(\rho_{\beta}^{2}\right)$ value of the prefactor (141) by considering the quantity

$$
\Gamma(T)=\frac{\xi-\left\langle\sigma_{\ell+1}^{x}(0) \sigma_{1}^{x}(0)\right\rangle e^{\ell \int_{-\pi}^{\pi} \frac{d x}{2 \pi} \log (1-4 \pi \rho(x))}}{\xi-C},
$$

where $C$ is given by 141 .
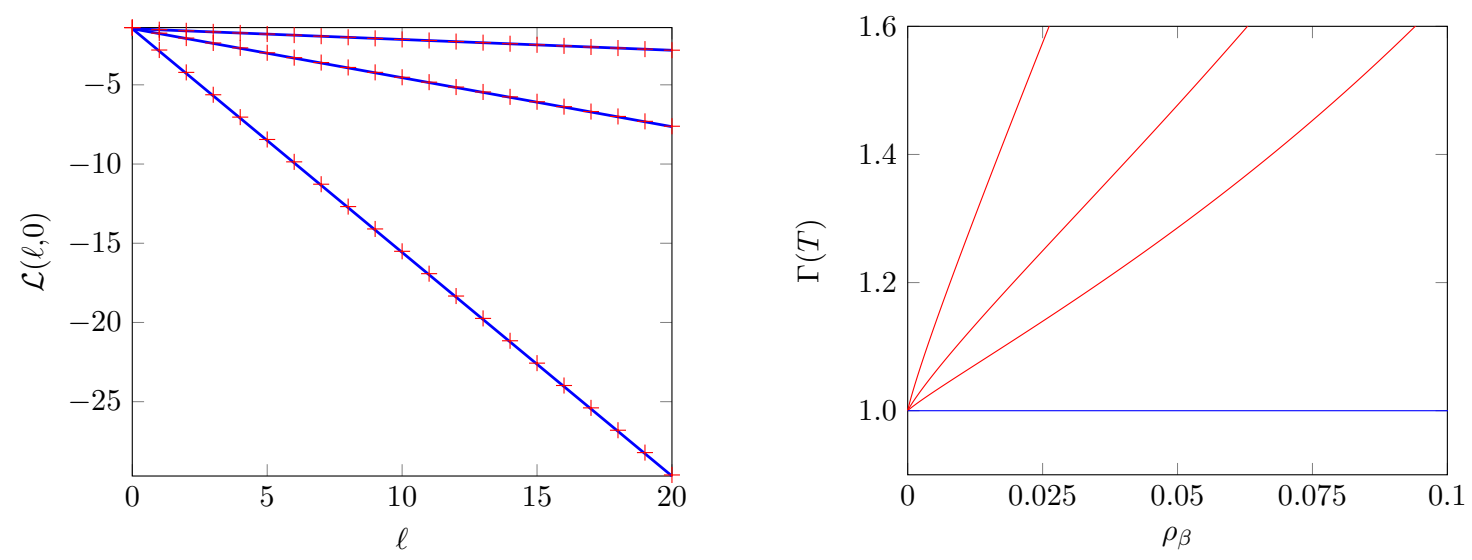

Figure 11: Left: $\mathcal{L}(\ell, 0)$ for $h=0.5$ and $\beta=2,1,0.25$ (top to bottom). Numerical results for a $L=200$ site open chain are shown as red crosses and eqn 140 by a continuous blue line. Right: Numerically determined $\Gamma(T)$ as a function of $\rho_{\beta}$ for $\ell=30$ and $h=$ $3 / 4,1 / 2,1 / 4$ from top to bottom; in blue is the expected value as $\rho_{\beta} \rightarrow 0$.

We see that $\Gamma(T)$ approaches 1 for small values of $T$, which means that the prefactor (141) is indeed correct to order $\mathcal{O}\left(\rho_{\beta}^{2}\right)$. The linear increase in $\rho_{\beta}$ shows that the correction to our result for $C$ is $\mathcal{O}\left(\rho_{\beta}^{3}\right)$.

We now turn to the time-like region. In Fig. 12 we compare numerical results for $\mathcal{L}(\ell, t)$ as a function of $t$ for different values of $\ell$ to 140 . We recall that in the time-like region eqn (140) only gives the leading time behaviour, i.e. the exponent of the exponential decay. Hence only the slope of our analytic result for $\mathcal{L}(\ell, t)$ has to match the numerics. This is indeed seen to be the case in Fig. 12. In the space-like regime, i.e. at sufficiently short times, 140 is again seen to be in very good agreement with the numerical results. 

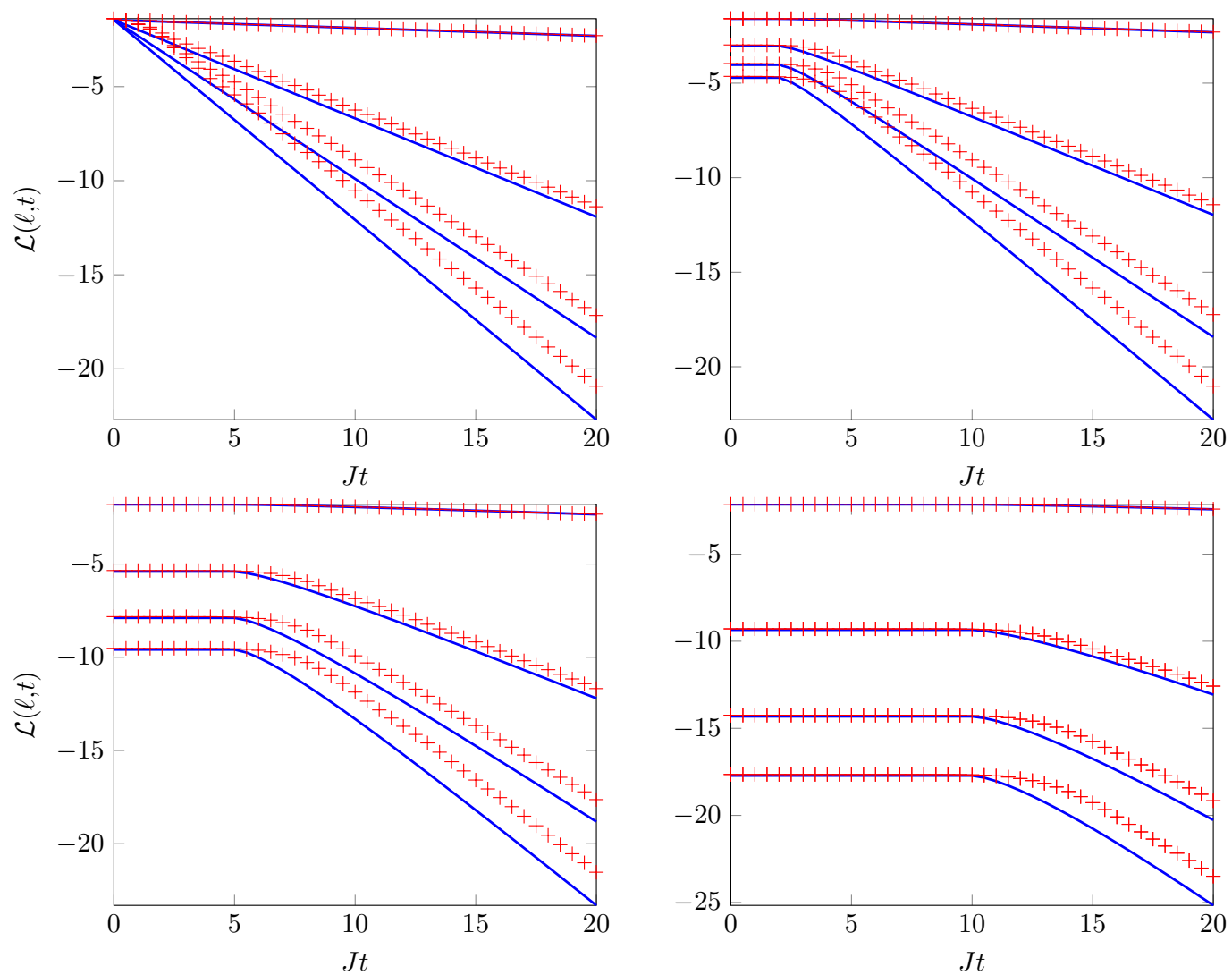

Figure 12: $\mathcal{L}(\ell, t)$ as a function of $t$ for $h=0.5$ and $\beta=2,0.5,0.286,0.2$ from top to bottom inside each panel, with $\ell=0,2,5,10$ from top left to bottom right. Numerical results for a $L=200$ site open chain are shown by red crosses and eqn 140 by a straight blue line. The slopes of the numerical results are correctly reproduced by $(140)$ in the time-like region, i.e. for $v_{\max } t>\ell$.

In order to have a more quantitative check on the exponential factor in 140 it is useful to consider the difference

$$
\mathcal{L}(\ell, t)-\log \chi^{x x}(\ell, t),
$$

where $\chi^{x x}(\ell, t)$ is given by 140 . If our exponential factor is exact, the difference should decay at late times more slowly than exponential, i.e. supposedly as a power law. In Figure 13 we plot (144) as a function of $\log J t$ and indeed observe a linear behaviour. This confirms that the exponential factor in (140) is exact and moreover establishes that the subleading asymptotics is power law in time. 

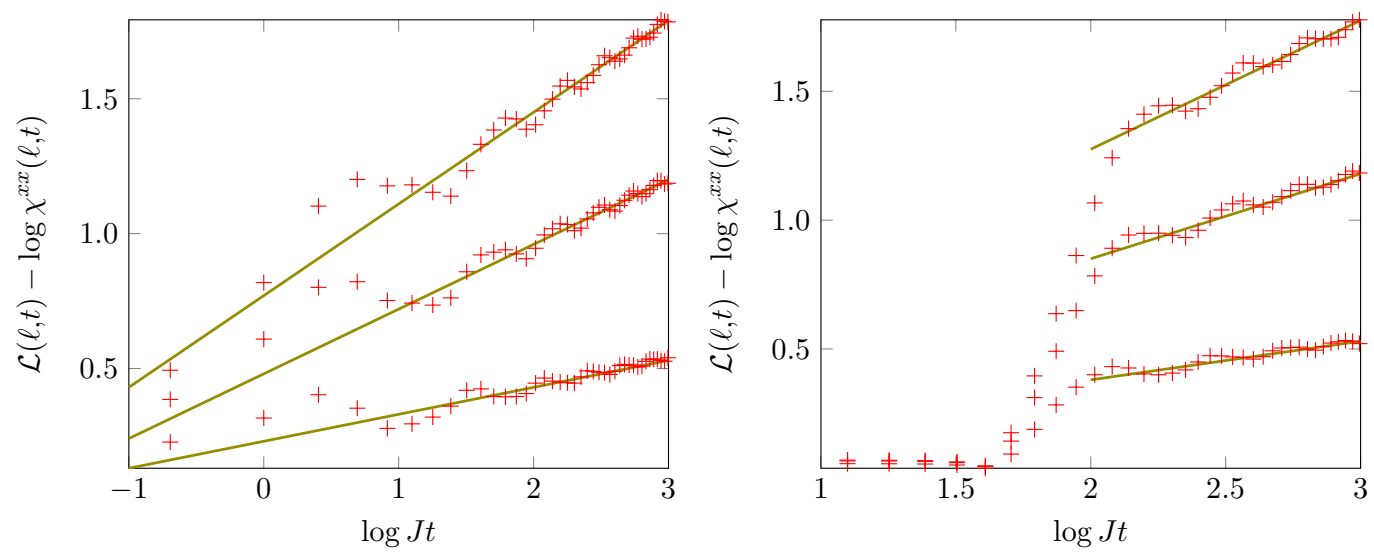

Figure 13: $\mathcal{L}(\ell, t)-\log \chi^{x x}(\ell, t)$ with $\chi^{x x}(\ell, t)$ given by 140$)$ as a function of $\log J t$ for $h=0.5$ and $\beta=0.5,0.286,0.2$ from bottom to top inside each panel, with $\ell=0$ (left) and $\ell=5$ (right) in red. In green is indicated a linear fit in the time-like region $v_{\max } t>\ell$.

\subsection{Disordered phase $h>1$}

\subsubsection{An approximate mapping to the ordered case}

In the disordered phase the form factors in (33) still differ from the finite temperature ordered case by the fact that there are no intermediate states with the same number of momenta as in the representative state. From section 3.2 we know that the terms $M=N+1$ and $M=N-1$ are equally important at late times, but the subleading terms are dominant in $\rho_{\beta}$ for $M=N+1$. Since we are interested in the leading time behaviour only, with the order $\rho_{\beta}^{0}$ subleading corrections, we will restrict our analysis to the case $M=N+1$.

In this case the partial fraction decomposition 690 of $\left.\left.\right|_{\mathrm{NS}}\left\langle q_{1}, \ldots, q_{N}\left|\sigma_{l}^{x}\right| p_{1}, \ldots, p_{M}\right\rangle_{\mathrm{R}}\right|^{2}$ necessarily involves one $\nu_{j}=0$. To obtain the $p_{j}$ dependence of the corresponding $A$ 's in (69), it suffices to decompose the form factor $\left.\left.\right|_{\mathrm{NS}}\left\langle q_{1}, \ldots, q_{N}\left|\sigma_{l}^{x}\right| p_{1}, \ldots, p_{M}\right\rangle_{\mathrm{R}}\right|^{2}$ by starting to write the partial fraction decomposition with respect to $p_{j}$ and retaining only the $\nu_{j}=0$ part (which corresponds to $P_{0}(X)$ in (36)), and decomposing with respect to the other momenta in the usual fashion. This part is $\frac{\prod_{i=1}^{N} \varepsilon_{p_{j} q_{i}}^{2}}{\prod_{i=1}^{N+1} \varepsilon_{p_{j} p_{i}}}$, that is $\frac{1}{\varepsilon\left(p_{j}\right)}$ times $\frac{\prod_{i=1}^{N} \varepsilon_{p_{j} q_{i}}^{2}}{\prod_{i=1 \neq j}^{N+1} \varepsilon_{p_{j} p_{i}}}$. This latter factor satisfies the hypotheses of 109 which allows us to replace it by 1 as long as we are interested only in the leading time behaviour. It follows that under this approximation the $\nu_{j}=0$ terms in the partial fraction decomposition 690 of $\left|{ }_{\mathrm{NS}}\left\langle q_{1}, \ldots, q_{N}\left|\sigma_{\ell}^{x}\right| p_{1}, \ldots, p_{M}\right\rangle_{\mathrm{R}}\right|^{2}$ contribute to (33) as

$$
\begin{aligned}
\left.\left.\frac{2 J \sqrt{h} \xi}{(N+1) ! L} \sum_{p_{j} \in \mathrm{R}} \frac{e^{-i t \bar{\varepsilon}\left(p_{j}\right)}}{\varepsilon\left(p_{j}\right)} \sum_{p_{i} \in \mathrm{R}, \mathrm{i} \neq \mathrm{j}}\right|_{\mathrm{R}}\left\langle p_{1}, \ldots, p_{j-1} p_{j+1}, \ldots, p_{N+1}\left|\sigma_{\ell}^{x}\right| q_{1}, \ldots, q_{N}\right\rangle_{\mathrm{NS}}\right|^{2} \\
\times e^{i t\left(E(\{q\})-E(\{p\})-\left\{p_{j}\right\}\right)+i \ell\left(P\left(\{p\}-\left\{p_{j}\right\}\right)-P(\{q\})\right)} .
\end{aligned}
$$

Taking into account all the possible $j=1, \ldots, N+1$ for which one can have $\nu_{j}=0$ in 69, 
we obtain

$$
\begin{aligned}
\chi^{x x}(\ell, t) \approx & \left(\frac{2 J \sqrt{h} \xi}{L} \sum_{p \in \mathrm{R}} \frac{e^{-i t \bar{\varepsilon}(p)}}{\varepsilon(p)}\right) \\
& \times\left.\left.\frac{1}{N !} \sum_{\substack{1 \\
\in \\
\in \mathrm{R}}}\right|_{\mathrm{R}}\left\langle p_{1}, \ldots, p_{N}\left|\sigma_{\ell}^{x}\right| q_{1}, \ldots, q_{N}\right\rangle_{\mathrm{NS}}\right|^{2} e^{i t(E(\{q\})-E(\{p\}))+i \ell(P(\{p\})-P(\{q\}))},
\end{aligned}
$$

where $\{p\}=\left\{p_{1}, \ldots, p_{N}\right\}$ has now the same number of momenta as in the representative state. The second factor in 146 is precisely of the same form as the one we considered in the ordered phase.

\subsubsection{Two-point dynamical correlation functions in the disordered phase}

Putting everything together we obtain the following result for the leading late time behaviour of the two-point function

$$
\chi^{x x}(\ell, t) \approx C(\ell, t) \exp \left(\frac{1}{2 \pi} \int_{-\pi}^{\pi}\left|t \varepsilon^{\prime}(x)+\ell\right| \log \tanh \frac{\beta \varepsilon(x)}{2} d x\right),
$$

where (cf. section 3.2 )

$$
C(\ell, t)= \begin{cases}2 J \sqrt{h} \xi \int_{-\pi}^{\pi}\left(\frac{e^{-i t \bar{\varepsilon}(x)}}{2 \pi}+2 \rho(x) \cos (t \bar{\varepsilon}(x))\right) \frac{d x}{\varepsilon(x)} & \text { if } v_{\max } t<\ell \text { at } \mathcal{O}\left(\rho_{\beta}\right) \\ 2 J \sqrt{h} \xi \int_{-\pi}^{\pi} \frac{e^{-i t \bar{\varepsilon}(x)}}{2 \pi \varepsilon(x)} d x & \text { if } v_{\max } t>\ell \text { at } \mathcal{O}\left(\rho_{\beta}^{0}\right)\end{cases}
$$

$l, t \geq 0$, and $v_{\max }$ is defined in $(35)$.

\subsubsection{Numerical checks}

We have checked the accuracy of (147) by comparing it to numerical calculations following Ref. [84]. In the following we show results for

$$
\mathcal{R}(\ell, t)=\operatorname{Re}\left(\left\langle\sigma_{\ell+1}^{x}(t) \sigma_{1}^{x}(0)\right\rangle\right) .
$$

In the space-like region $v_{\text {max }} t<\ell$ we furthermore check our result for the prefactor $C(\ell, t)$ (148) by computing

$$
\Lambda(\ell, T)=\frac{\mathcal{R}(\ell, 0) e^{-\ell \int_{-\pi}^{\pi} \frac{d x}{2 \pi} \log \tanh \left(\frac{\beta \varepsilon(x)}{2}\right)}-C_{0}(\ell, 0)}{C(\ell, 0)-C_{0}(\ell, 0)},
$$

where $C_{0}(\ell, t)=\frac{J \sqrt{h} \xi}{\pi} \int_{-\pi}^{\pi} \frac{d x}{\varepsilon(x)} e^{-i t \bar{\varepsilon}(x)}$ is the $\mathcal{O}\left(\rho_{\beta}^{0}\right)$ contribution to $C(\ell, t)$. If and only if the prefactor in 148 is correct at order $\mathcal{O}\left(\rho_{\beta}\right), C(\ell, t) \rightarrow 1$ when $T \rightarrow 0$.

In Fig. 14 we compare our analytic expression (147) in the space-like region to numerical results for $R(\ell, t)$. 

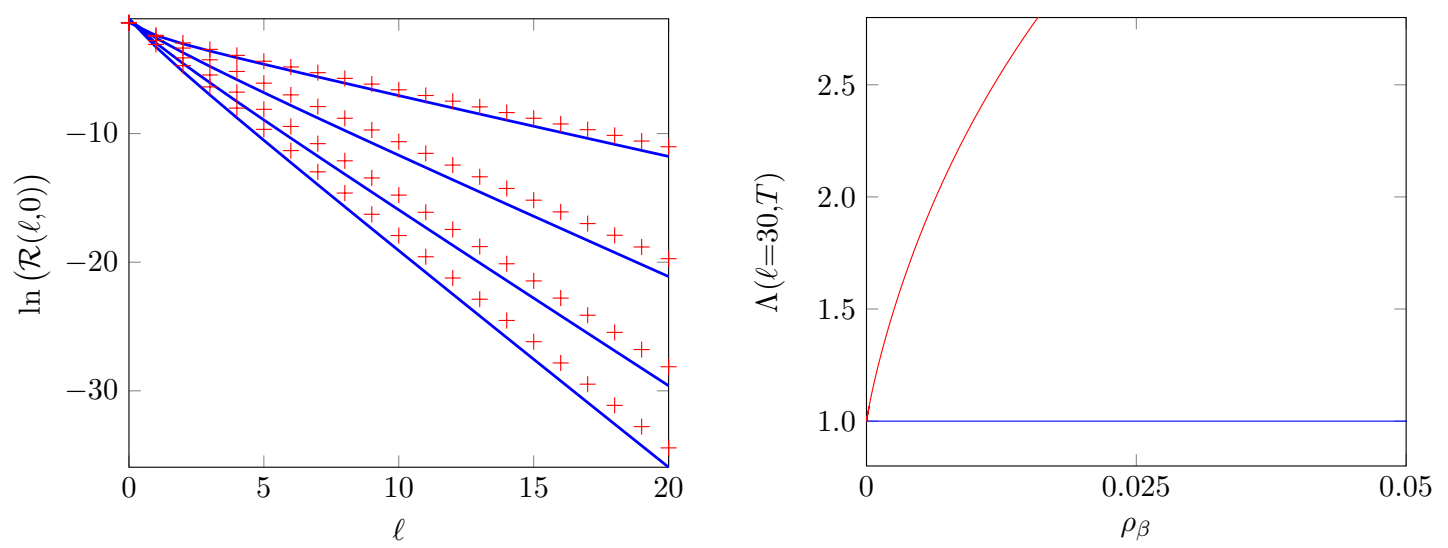

Figure 14: Left: $\log (\mathcal{R}(\ell, 0))$ as a function of $\ell$ for $h=1.5$ and $\beta=2,0.5,0.285,0.2$ from top to bottom. Numerical results for a $L=200$ site open chain are shown as red crosses and eqn (147) as straight blue lines. Right: numerical results for $\Lambda(\ell=30, T)$ as a function of $\rho_{\beta}$ for $h=3 / 2$ (red line). The zero temperature result $\Lambda(\ell=30,0)=1$ is shown for comparison.

Our analytic expression is seen to be in good agreement with the numerical results and the remaining discrepancy is due to $\mathcal{O}\left(\rho_{\beta}^{2}\right)$ corrections to the prefactor.

In Fig. 15 we present results for $\mathcal{R}(\ell, t)$ in the time-like region $v_{\max } t>\ell$ at low temperatures. We see that the analytical result is in excellent agreement with the numerics.
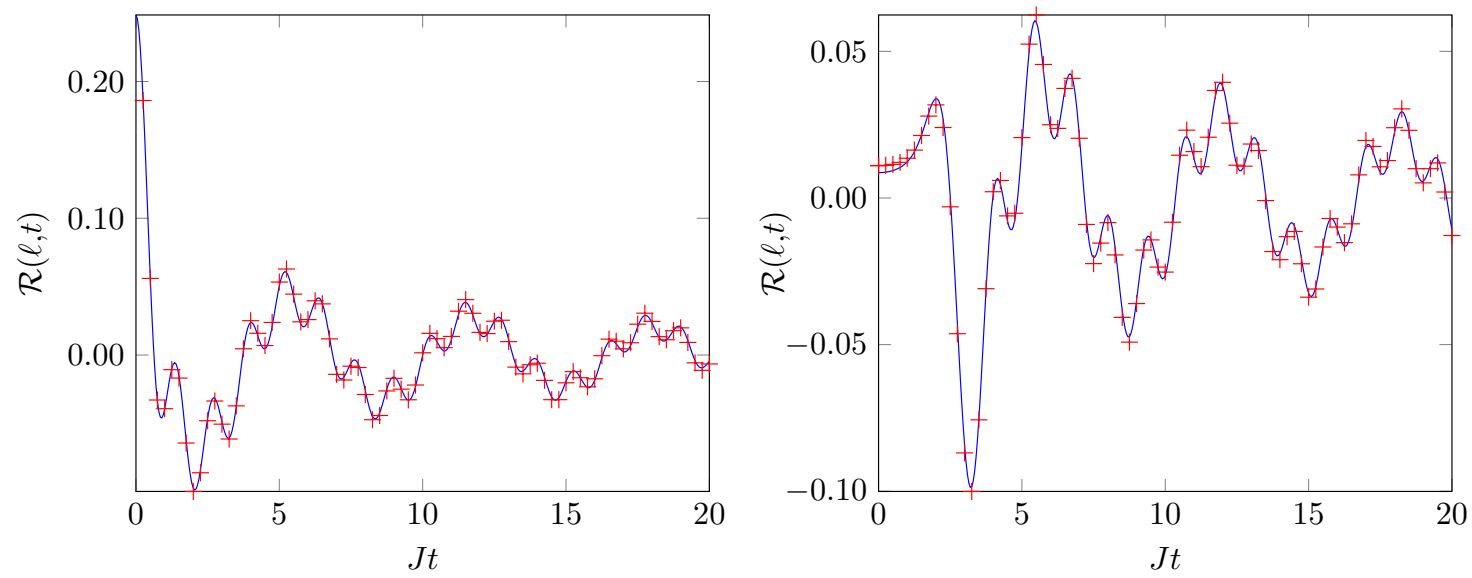

Figure 15: $\mathcal{R}(\ell, t)$ as a function of $t$ with $h=1.5$ and $\beta=3$ for $\ell=0$ (left) and $\ell=5$ (right). Numerical results for a $L=200$ site open chain are shown as red crosses and eqn (147) as a solid blue line.

In order to check the accuracy of our result for the exponential decay of the two-point function for intermediate and high temperatures we compare (147) to numerical results $\ln |\mathcal{R}(\ell, t)|$ in Fig. 16. As our result for the prefactor $C(\ell, t)$ only holds at low temperatures we expect the numerical results to differ from our analytical prediction by an essentially constant offset. This is indeed what we observe. 

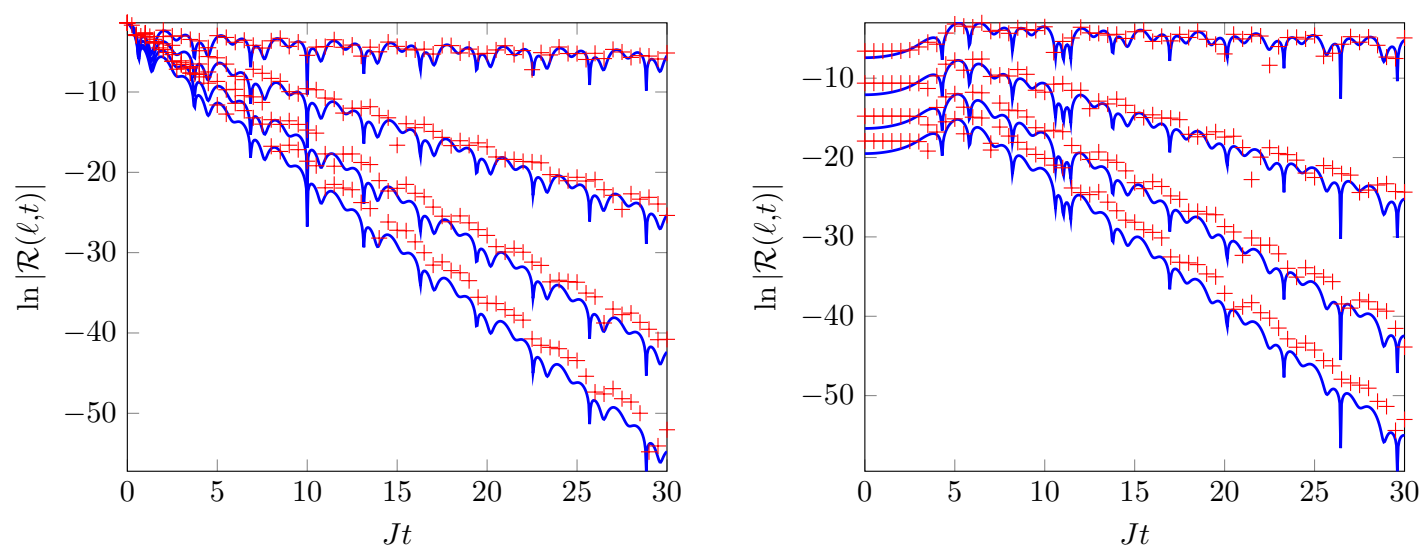

Figure 16: $\log |\mathcal{R}(\ell, t)|$ as a function of $t$ for $h=0.5$ and $\beta=2,0.5,0.286,0.2$ from top to bottom inside each panel, with $\ell=0$ (left) and $\ell=10$ (right). Numerical results for a $L=200$ site open chain are shown as red crosses and eqn (147) as a solid blue line.

\section{Summary and Discussion}

In this work we have considered two problems in the transverse field Ising model: (i) The time dependence of the order parameter after a quantum quench; (ii) The dynamical order parameter two point function in equilibrium at finite temperatures. Using the quench action approach for (i) and a micro-canonical formulation combined with typicality ideas for (ii) these problems can both be formulated in terms of spectral representations using Hamiltonian eigenstates, i.e. sums over form factors.

These highly intricate sums over a macroscopic quantity of momenta of a form factor with many singularities represent however a considerable technical challenge. We showed that in the case of semi-local operators such as $\sigma_{j}^{x}$ in the TFIM, this difficulty can be addressed by decomposing the form factor into partial fractions, which permits the sums over momenta to be decoupled and the late time behaviour to be determined. These partial fractions can be organized in terms of the degree and the position of theirs poles, which naturally leads to an expansion in the density of particles of the representative state. The leading behaviour at late times can be then computed at all orders in the density through a determinant representation of these poles, which leads invariably to an exponential decay.

Our analysis provides a precise characterization of the excitations over the representative state that contributes to the late time behaviour of correlation functions of semi-local operators. We find that simultaneous particle-hole excitations of all particles in the representative state contribute to the correlation function, and we altogether have to sum over $\mathcal{O}\left((\epsilon L)^{N}\right)$ excited states, where $\epsilon$ is a fixed number that can be taken as small as desired and $N / L$ is the density. In particular, this implies that the appealing picture of an expansion in terms of a finite number of particle-hole excitations over the full momentum space fails for semi-local operators at finite temperatures. The form factor sum is dominated by mesoscopic excitations (in the sense given in section 3.5.1) around each single particle, which is an exponential number of states, but subentropic in the sense that includes only states whose macroscopic state is the representative state itself.

We have compared our analytic results to numerical computations in both the finite temperature and quench contexts. In the absence of saddle points, thus where the sums (43) are valid at all momenta, we find remarkable agreement at all times. In cases where 
saddle points occur our numerical results indicate the presence of a multiplicative powerlaw behaviour as a subleading correction to the exponential decay. We believe that this will emerge from saddle point effects of these classes of excitations, due to the fact that (43) is not valid anymore close to the saddle point. Higher-order corrections in time will also arise from contributions with $\nu_{i}=0$ in (39); in this case there is no singularity and the full momentum space should be summed over to take them into account. We believe that partial fraction decompositions for form factors of semi-local operators are not only suited for extracting the late time asymptotics, but should also be useful for determining intermediate and short time behaviours.

In interacting models, the singularity structure of the form factors of semi-local operators is not fundamentally changed and a partial fraction decomposition will provide a useful organizing principle as well. A notable difference is that the Bethe equations link the different particles so that the momenta sums can never fully decouple. This will be the subject of a subsequent paper.

For local operators however, the story is radically different. The singularity structure of the form factors $(12)$ is completely dissimilar and one cannot use partial fraction decomposition related to an expansion in powers of particle density to extract the late time behaviour. Excitations over the full momentum space and not only near the singularities will play equally important roles. This will be discussed elsewhere.

Acknowledgements We are grateful to Jacopo de Nardis and Frank Göhmann for helpful discussions. This work was supported by the EPSRC under grant EP/S020527/1 (FHLE and EG) and by the ERC under Starting Grant No. 805252 LoCoMacro (MF).

\section{A Riemann sums of singular functions}

Sums of form factors lead to Riemann sums of functions with a quadratic singularity of the form

$$
\frac{1}{L} \sum_{k=-L+1}^{L} f\left(\frac{k}{L}\right) \quad \text { with } f(x) \underset{x \rightarrow 0}{\sim} \frac{1}{x^{2}} .
$$

Since the integral of $f(x)$ is divergent, the limit $L \rightarrow \infty$ cannot be directly taken as for regular functions and needs special treatment. The purpose of this appendix is to explain techniques to compute them.

\section{A.1 Oscillatory sums}

Oscillatory Riemann sums of functions with a quadratic singularity cannot be estimated with the usual results on stationary phase approximation to obtain their large time behaviour. The principle is then to add and remove an elementary function with the same singularity, but whose Riemann sum can be computed directly, so that the remaining function has no singularity and thus has an oscillatory Riemann sum that vanishes at large times.

\section{A.1.1 Generic example}

For concreteness, let us illustrate this procedure with the Riemann sum

$$
S_{L}(\theta)=\frac{1}{L} \sum_{k=-L+1 \neq 0}^{L} f\left(\frac{k}{L}\right) e^{i \frac{k}{L} \theta},
$$


for $f(x)$ a function such that $f(x)=\frac{1}{x^{2}}+\mathcal{O}(1)$ for $x \rightarrow 0, f$ being regular otherwise. Then

$$
S_{L}(\theta)=\frac{1}{L} \sum_{k=-L+1 \neq 0}^{L}\left(\frac{L}{k}\right)^{2} e^{i \frac{k}{L} \theta}+\frac{1}{L} \sum_{k=-L+1}^{L} \bar{f}\left(\frac{k}{L}\right) e^{i \frac{k}{L} \theta},
$$

The second term on the right-hand side can be turned into an integral, while we rewrite the first as follows

$$
\frac{1}{L} \sum_{k=-L+1 \neq 0}^{L}\left(\frac{L}{k}\right)^{2} e^{i \frac{k}{L} \theta}=L \sum_{k=-\infty, \neq 0}^{+\infty} \frac{e^{i \frac{k}{L} \theta}}{k^{2}}-\frac{1}{L} \sum_{k=L+1}^{+\infty}\left(\frac{L}{k}\right)^{2} e^{i \frac{k}{L} \theta}-\frac{1}{L} \sum_{k=-\infty}^{-L}\left(\frac{L}{k}\right)^{2} e^{i \frac{k}{L} \theta} .
$$

The second and third Riemann sums can now be turned into integrals without any problems, which gives

$$
S_{L}(\theta)=L \sum_{k=-\infty, \neq 0}^{+\infty} \frac{e^{i \frac{k}{L} \theta}}{k^{2}}+\int_{-1}^{1} \bar{f}(x) e^{i \theta x} d x-\int_{1}^{\infty} \frac{e^{i \theta x}}{x^{2}} d x-\int_{-\infty}^{-1} \frac{e^{i \theta x}}{x^{2}} d x+\mathcal{O}\left(L^{-1}\right) .
$$

The three integrals are oscillatory integrals of bounded functions and hence vanish for large $\theta$. We conclude that

$$
S_{L}(\theta)=L \sum_{k=-\infty, \neq 0}^{+\infty} \frac{e^{i \frac{k}{L} \theta}}{k^{2}}+\mathcal{O}\left(L^{0} \theta^{-1 / 2}\right)+\mathcal{O}\left(L^{-1}\right)
$$

\section{A.1.2 Elementary oscillatory sums with singularities}

The above analysis shows that the leading asymptotics of Riemann sums with simple or double poles involves the following sums

$$
\begin{aligned}
& \sum_{n \in \mathbb{Z}} \frac{e^{i w(n+\alpha)}}{(n+\alpha)}=\frac{\pi}{\sin \pi \alpha} e^{i \pi \alpha \operatorname{sgn}(w)}, \quad \text { for }-\pi<w \leq \pi \\
& \sum_{n \in \mathbb{Z}} \frac{e^{i w(n+\alpha)}}{(n+\alpha)^{2}}=\left(\frac{\pi}{\sin \pi \alpha}\right)^{2}+\frac{i \pi}{\sin \pi \alpha} w e^{i \pi \alpha \operatorname{sgn}(w)}, \quad \text { for }-\pi<w \leq \pi .
\end{aligned}
$$

These are readily obtained by computing the Fourier series coefficients of the right-hand sides multiplied by $e^{-i w \alpha}$ and seen as a $2 \pi$-periodic function of $w$. In particular we have

$$
\begin{gathered}
\sum_{n \in \mathbb{Z}} \frac{e^{i w(n+1 / 2)}}{(n+1 / 2)^{m}}= \begin{cases}\pi^{2}\left(1-\frac{|w|}{\pi}\right) & \text { if } m=2, \\
i \pi \operatorname{sgn}(w) & \text { if } m=1 .\end{cases} \\
\sum_{n \in \mathbb{Z} \backslash\{0\}} \frac{e^{i w n}}{n^{m}}= \begin{cases}\pi^{2}\left(\frac{1}{3}-\frac{|w|}{\pi}+\frac{w^{2}}{2 \pi^{2}}\right) & \text { if } m=2, \\
i(\pi-|w|) \operatorname{sgn}(w) & \text { if } m=1 .\end{cases}
\end{gathered}
$$

\section{A.1.3 Equations 43}

Following the steps outlined above we obtain for $q \in \mathrm{NS}$

$$
\begin{aligned}
\sum_{p \in \mathrm{R}} \frac{e^{-i t \bar{\varepsilon}(p)}}{L \sin ^{2}\left(\frac{p-q}{2}\right)}= & L \sum_{k=-\infty}^{+\infty} \frac{e^{-i t \bar{\varepsilon}(q+2 \pi(k+1 / 2) / L)}}{\pi^{2}(k+1 / 2)^{2}}-\int_{\pi}^{\infty} \frac{4 e^{-i t \bar{\varepsilon}(x)}}{(x-q)^{2}} \frac{d x}{2 \pi}-\int_{-\infty}^{-\pi} \frac{4 e^{-i t \bar{\varepsilon}(x)}}{(x-q)^{2}} \frac{d x}{2 \pi} \\
& +\int_{-\pi}^{\pi} e^{-i t \bar{\varepsilon}(x)}\left(\frac{1}{\sin ^{2} \frac{x-q}{2}}-\frac{1}{(x-q)^{2} / 4}\right) \frac{d x}{2 \pi}+\mathcal{O}\left(L^{-1}\right) .
\end{aligned}
$$


At leading order in time, one can Taylor expand the $\bar{\varepsilon}$ in the remaining sum to fall back on an elementary oscillatory sum. The three integrals involve oscillatory bounded functions and hence decay to zero with time.

Similarly we obtain for $q \in$ NS

$$
\begin{aligned}
\sum_{p \in \mathrm{R}} \frac{e^{-i t \bar{\varepsilon}(p)}}{L \sin \left(\frac{p-q}{2}\right)} & =\sum_{k=-\infty}^{+\infty} \frac{e^{-i t \bar{\varepsilon}(q+2 \pi(k+1 / 2) / L)}}{\pi(k+1 / 2)}-\int_{\pi}^{\infty} \frac{2 e^{-i t \bar{\varepsilon}(x)}}{(x-q)} \frac{d x}{2 \pi}-\int_{-\infty}^{-\pi} \frac{2 e^{-i t \bar{\varepsilon}(x)}}{(x-q)} \frac{d x}{2 \pi} \\
& +\int_{-\pi}^{\pi} e^{-i t \bar{\varepsilon}(x)}\left(\frac{1}{\sin \frac{x-q}{2}}-\frac{1}{(x-q) / 2}\right) \frac{d x}{2 \pi}+\mathcal{O}\left(L^{-1}\right)
\end{aligned}
$$

where the integrals are again integrals of oscillatory bounded functions and vanish at late times.

Finally we use (158) to obtain the asymptotic values of the sums in 159 ) and (160) for $q \in$ NS and $\bar{\varepsilon}^{\prime}(q) \neq 0$

$$
\begin{gathered}
\sum_{p \in \mathrm{R}} \frac{e^{-i t \bar{\varepsilon}(p)}}{L \sin \left(\frac{p-q}{2}\right)}=-i \operatorname{sgn}\left(t \bar{\varepsilon}^{\prime}(q)\right) e^{-i t \bar{\varepsilon}(q)}+\mathcal{O}\left(L^{0} t^{-1 / 2}\right)+\mathcal{O}\left(L^{-1}\right), \\
\sum_{p \in \mathrm{R}} \frac{e^{-i t \bar{\varepsilon}(p)}}{L^{2} \sin ^{2}\left(\frac{p-q}{2}\right)}=\left(1-\frac{2\left|t \bar{\varepsilon}^{\prime}(q)\right|}{L}\right) e^{-i t \bar{\varepsilon}(q)}+\mathcal{O}\left(L^{-1} t^{-1 / 2}\right)+\mathcal{O}\left(L^{-2}\right) .
\end{gathered}
$$

\section{A.1.4 Equations 86}

We now turn to momentum sums of the form

$$
\Sigma^{(n)}\left(q, q^{\prime}, t\right)=\frac{4}{L^{n}} \sum_{0<p \in \mathrm{R}} \frac{\sin p \sin q^{\prime} f(p)}{(\cos q-\cos p)^{n} f\left(q^{\prime}\right)} e^{2 i t(\varepsilon(p)-\varepsilon(q))}, \quad n=1,2,
$$

where $f(q)$ is defined in 83 . Using that $\frac{\sin x f(x)}{\cos q-\cos x}-\frac{f(q)}{x-q}$ is a bounded function of $x$ we can proceed along the same lines as in subsection A.1.3 to conclude that for $q \in$ NS

$$
\Sigma^{(1)}\left(q, q^{\prime}, t\right)=\frac{4}{L} \sum_{0<p \in \mathrm{R}} \frac{\sin q^{\prime}}{p-q} e^{2 i t(\varepsilon(p)-\varepsilon(q))} \frac{f(q)}{f\left(q^{\prime}\right)}+\mathcal{O}\left(L^{0} t^{-1 / 2}\right) .
$$

At leading order in time we may then Taylor expand $\varepsilon(p)$ around $p=q$, and write $p=q+\frac{2 \pi}{L}(n+1 / 2)$ to fall back on one of the oscillatory sums in 158$)$. In this way we obtain our final result

$$
\Sigma^{(1)}\left(q, q^{\prime}, t\right)=2 i \operatorname{sgn}\left(t \varepsilon^{\prime}(q)\right) \sin q^{\prime} \frac{f(q)}{f\left(q^{\prime}\right)}+\mathcal{O}\left(L^{0} t^{-1 / 2}\right) .
$$

The analysis of the $\Sigma^{(2)}\left(q, q^{\prime}, t\right)$ proceeds in complete analogy: we use that $\frac{\sin x f(x)}{(\cos x-\cos q)^{2}}-$ $\frac{f(q)}{\sin q(x-q)^{2}}-\frac{f^{\prime}(q)}{\sin q(x-q)}$ is a bounded function of $x$ to conclude that

$$
\Sigma^{(2)}\left(q, q^{\prime}, t\right)=\frac{4}{L^{2}} \sum_{p>0, \in \mathrm{R}} \frac{e^{2 i t(\varepsilon(p)-\varepsilon(q))}}{(p-q)^{2}}+\frac{4}{L^{2}} \sum_{p>0, \in \mathrm{R}} \frac{f^{\prime}(q)}{f(q)} \frac{e^{2 i t(\varepsilon(p)-\varepsilon(q))}}{p-q}+\mathcal{O}\left(L^{-1} t^{-1 / 2}\right) .
$$

Taylor expanding $\varepsilon(p)$ around $p=q$ and using (158) we finally arrive at

$$
\Sigma^{(2)}\left(q, q^{\prime}, t\right)=1-\frac{4\left|t \varepsilon^{\prime}(q)\right|}{L}+\frac{2 i \operatorname{sgn}\left(t \varepsilon^{\prime}(q)\right)}{L} \frac{f^{\prime}(q)}{f(q)}+\mathcal{O}\left(L^{-1} t^{-1 / 2}\right) .
$$




\section{A.1.5 Two-dimensional sums}

In this subsection we apply the method described above to the calculation of the twodimensional sums arising in section 3.1.5. We first consider

$$
\Omega_{1}(t)=-\frac{8}{L^{2}} \sum_{i \neq j} \frac{\sin q_{i} \sin q_{j} \frac{f\left(q_{i}\right)}{f\left(q_{j}\right)}}{\left(\cos q_{i}-\cos q_{j}\right)^{2}} e^{2 i t\left(\varepsilon\left(q_{i}\right)-\varepsilon\left(q_{j}\right)\right)} .
$$

where for large $L$ the $q$ 's are distributed according to the density $\rho(q)$. Starting with the sum over $q_{i}$ and using that $\frac{\sin x f(x)}{(\cos x-\cos q)^{2}}-\frac{f(q)}{\sin q(x-q)^{2}}-\frac{f^{\prime}(q)}{\sin q(x-q)}$ is a bounded function of $x$, we conclude that

$$
\Omega_{1}(t)=-\frac{8}{L^{2}} \sum_{i \neq j} \frac{e^{2 i t\left(\varepsilon\left(q_{i}\right)-\varepsilon\left(q_{j}\right)\right)}}{\left(q_{i}-q_{j}\right)^{2}}-\frac{8}{L^{2}} \sum_{i \neq j} \frac{f^{\prime}\left(q_{j}\right)}{f\left(q_{j}\right)} \frac{e^{2 i t\left(\varepsilon\left(q_{i}\right)-\varepsilon\left(q_{j}\right)\right)}}{q_{i}-q_{j}}+\mathcal{O}\left(t^{-\frac{1}{2}} L^{0}\right) .
$$

Next we Taylor expand $\varepsilon\left(q_{j}\right)$ around $q_{j}=q_{i}$ and use

$$
q_{j}-q_{i}=\frac{j-i}{L \rho\left(q_{j}\right)}+o\left(L^{-1}\right),
$$

to obtain

$$
\Omega_{1}(t)=-8 \sum_{j \neq i} \frac{e^{i \vartheta_{j}(i-j)}}{(i-j)^{2}} \rho^{2}\left(q_{j}\right)-\frac{8}{L} \sum_{j \neq i} \frac{e^{i \vartheta_{j}(i-j)}}{i-j} \rho\left(q_{j}\right) \frac{f^{\prime}\left(q_{j}\right)}{f\left(q_{j}\right)}+\mathcal{O}\left(t^{-\frac{1}{2}} L^{0}\right),
$$

where $\vartheta_{j}=2 t \varepsilon^{\prime}\left(q_{j}\right) /\left(L \rho\left(q_{j}\right)\right)$. The sums over $i$ can then be carried out using (158)

$$
\Omega_{1}(t)=-8 \sum_{j} \rho^{2}\left(q_{j}\right)\left[\frac{\pi^{2}}{3}-\pi \vartheta_{j}\right]-\frac{8 \pi i}{L} \sum_{j} \rho\left(q_{j}\right) \frac{f^{\prime}\left(q_{j}\right)}{f\left(q_{j}\right)}+\mathcal{O} L^{0}\left(t^{-1 / 2}\right) .
$$

Finally we turns sums into integrals and use 89 to obtain

$$
\Omega_{1}(t)=-L \frac{8 \pi^{2}}{3} \int_{0}^{\pi} \rho(x)^{3} d x+16 \pi \int_{0}^{\pi} \rho(x)^{2}\left|t \bar{\varepsilon}^{\prime}(x)\right| d x+\mathcal{O}\left(t^{-\frac{1}{2}} L^{0}\right) .
$$

Next we consider

$$
\Omega_{2}(t)=\frac{1}{L^{2}} \sum_{i \neq j} \frac{e^{i t\left(\bar{\varepsilon}\left(q_{j}\right)-\bar{\varepsilon}\left(q_{i}\right)\right)}}{\sin ^{2}\left(\frac{q_{i}-q_{j}}{2}\right)} \operatorname{sgn}\left(\bar{\varepsilon}^{\prime}\left(q_{j}\right) \bar{\varepsilon}^{\prime}\left(q_{i}\right)\right),
$$

The sum over $j$ can be carried out by proceeding in the same way as for $\Omega_{1}(t)$, which gives

$$
\Omega_{2}(t)=\frac{1}{L} \sum_{i} L \frac{\left(2 \pi \rho\left(q_{i}\right)\right)^{2}}{3}-4 \pi \rho\left(q_{i}\right)\left|t \bar{\varepsilon}^{\prime}\left(q_{i}\right)\right|+\mathcal{O}\left(L^{0} t^{-1 / 2}\right)+\mathcal{O}\left(L^{-1}\right) .
$$

Finally we turn sums into integrals to arrive at our final result

$$
\Omega_{2}(t)=L \frac{4 \pi^{2}}{3} \int_{-\pi}^{\pi} \rho(x)^{3} d x-4 \pi \int_{-\pi}^{\pi} \rho(x)^{2}\left|t \bar{\varepsilon}^{\prime}(x)\right| d x+\mathcal{O}\left(L^{0} t^{-1 / 2}\right)+\mathcal{O}\left(L^{-1}\right) .
$$




\section{A.2 Non-oscillatory sums}

We saw in the previous section that the non-decaying part of an oscillatory Riemann sum with singularity only depends on the singularity. However, if it is not oscillatory the Riemann sum will depend on the full function and its calculation is a bit more subtle. The principle is to separate the sum over two regions, one that is over points at distance less than $A L$ from the singularity, and the other one at distance larger than $A L$, for a fixed arbitrary $A>0$. By adding and removing the corresponding singularity in the first sum, one can take the limit $L \rightarrow \infty$ and obtain the result as a seemingly $A$-dependent but in fact constant expression. To obtain a simpler expression for this constant one then typically looks at the limit $A \rightarrow 0$ of the $A$-dependent expression.

\section{A.2.1 Generic example}

Let us again illustrate the procedure with an example:

$$
S_{L}=\frac{1}{L} \sum_{k=-L+1 \neq 0}^{L} f\left(\frac{k}{L}\right)
$$

for $f(x)$ a function such that $f(x)=\frac{1}{x^{2}}+\mathcal{O}(1)$ for $x \rightarrow 0, f$ being regular otherwise. Let us fix $A>0$ and decompose

$$
S_{L}=\frac{1}{L} \sum_{\substack{k=-L+1 \\|k|>A L}}^{L} f\left(\frac{k}{L}\right)+\frac{1}{L} \sum_{k=-A L+1}^{A L} \bar{f}\left(\frac{k}{L}\right)+\frac{1}{L} \sum_{k=-A L+1 \neq 0}^{A L}\left(\frac{L}{k}\right)^{2} .
$$

with $\bar{f}(x)=f(x)-1 / x^{2}$ a regular function. Using $\sum_{k=1}^{B} k^{-2}=\sum_{k=1}^{\infty} k^{-2}-B^{-1}+\mathcal{O}\left(B^{-2}\right)$, one obtains at fixed $A$ and $L \rightarrow \infty$

$$
S_{L}=\int_{A<|x|<1} f(x) d x+\int_{0<|x|<A} \bar{f}(x) d x+L \sum_{k=-\infty, \neq 0}^{+\infty} \frac{1}{k^{2}}-\frac{2}{A}+\mathcal{O}\left(L^{-1}\right) .
$$

Then, writing $f(x)=\left(x^{2} f(x)\right) / x^{2}$, integrating by part the first integral

$$
\begin{aligned}
S_{L}= & \int_{A<|x|<1} \frac{\left(x^{2} f(x)\right)^{\prime}}{x} d x-f(1)-f(-1)+A f(A)+A f(-A) \\
& +\int_{0<|x|<A} \bar{f}(x) d x+L \frac{\pi^{2}}{3}-\frac{2}{A}+\mathcal{O}\left(L^{-1}\right) .
\end{aligned}
$$

We note that the second integral involves a function that is bounded for $|x| \leq A$ and so vanishes for $A \rightarrow 0$. Since the relation holds for any $A>0$, in particular for $A \rightarrow 0$ we get

$$
S_{L}=L \frac{\pi^{2}}{3}+\int_{-1}^{1} \frac{\left(x^{2} f(x)\right)^{\prime}}{x} d x-f(1)-f(-1)+\mathcal{O}\left(L^{-1}\right)
$$

\section{A.2.2 Two-dimensional sums}

We now apply these ideas to the calculation of

$$
\Sigma_{0} \equiv \frac{1}{L^{2}} \sum_{i \neq j} \frac{1}{\sin ^{2}\left(\frac{q_{i}-q_{j}}{2}\right)} \operatorname{sgn}\left(\bar{\varepsilon}^{\prime}\left(q_{j}\right) \bar{\varepsilon}^{\prime}\left(q_{i}\right)\right)
$$


in the space-like region, where $\operatorname{sgn}\left(\bar{\varepsilon}^{\prime}\left(q_{j}\right) \bar{\varepsilon}^{\prime}\left(q_{i}\right)\right)=1$ everywhere. We first decompose the sum into divergent and finite parts

$$
\begin{aligned}
\Sigma_{0} & =4 \sum_{i \neq j} \frac{\rho\left(q_{i}\right)^{2}}{(i-j)^{2}}+\frac{1}{L^{2}} \sum_{i \neq j} \frac{1}{\sin ^{2}\left(\frac{q_{i}-q_{j}}{2}\right)}-\frac{4 L^{2} \rho\left(q_{i}\right)^{2}}{(i-j)^{2}} \\
& =\frac{4 \pi^{2}}{3} \sum_{j} \rho\left(q_{j}\right)^{2}+\frac{1}{L^{2}} \sum_{i \neq j} \frac{1}{\sin ^{2}\left(\frac{q_{i}-q_{j}}{2}\right)}-\frac{4 L^{2} \rho\left(q_{i}\right)^{2}}{(i-j)^{2}} .
\end{aligned}
$$

We evaluate the second term by splitting it into contributions from $\left|q_{i}-q_{j}\right|>B$ and $\left|q_{i}-q_{j}\right|<B$. The latter vanishes in the limit $B \rightarrow 0$, while the former becomes

$$
\begin{aligned}
& \frac{1}{L^{2}} \sum_{j} \sum_{|i-j|>B L \rho\left(q_{j}\right)} \frac{1}{\sin ^{2}\left(\frac{q_{i}-q_{j}}{2}\right)}-\frac{8}{B L} \sum_{j} \rho\left(q_{j}\right)+\mathcal{O}\left(L^{-1}\right) \\
& =\iint_{|x-y|>B} \frac{\rho(x) \rho(y)}{\sin ^{2} \frac{x-y}{2}} d x d y-\frac{8}{B} \int \rho(x)^{2} d x+\mathcal{O}(B)+\mathcal{O}\left(L^{-1}\right) \\
& =-2 \iint_{|x-y|>B} \frac{\rho(x) \rho^{\prime}(y)}{\tan \frac{x-y}{2}} d x d y+\mathcal{O}(B)+\mathcal{O}\left(L^{-1}\right)
\end{aligned}
$$

where we used in the first line $\sum_{k=1}^{B L} k^{-2}=\pi^{2} / 6-(B L)^{-1}+\mathcal{O}\left(L^{-2}\right)$, and where in the last line we have integrated by parts. Finally we take $B \rightarrow 0$ to obtain

$$
\Sigma_{0}=L \frac{4 \pi^{2}}{3} \int_{-\pi}^{\pi} \rho(x)^{3} d x-2 \int_{-\pi}^{\pi} \int_{-\pi}^{\pi} \frac{\rho(x) \rho^{\prime}(y)}{\tan \frac{x-y}{2}} d x d y+\mathcal{O}\left(L^{-1}\right) .
$$

Next we consider the sum

$$
\Sigma_{1}=\frac{8}{L^{2}} \sum_{i \neq j} \frac{\sin q_{i} \sin q_{j}}{\left(\cos q_{i}-\cos q_{j}\right)^{2}} .
$$

We again separate it into divergent and finite parts

$$
\begin{aligned}
\Omega_{1} & =8 \sum_{l \neq j} \frac{\rho^{2}\left(q_{j}\right)}{(l-j)^{2}}+\frac{8}{L^{2}} \sum_{l \neq j}\left[\frac{\sin q_{l} \sin q_{j}}{\left(\cos q_{j}-\cos q_{l}\right)^{2}}-\frac{L^{2} \rho^{2}\left(q_{j}\right)}{(l-j)^{2}}\right] \\
& =\frac{8 \pi^{3}}{3} \sum_{j} \rho^{2}\left(q_{j}\right)+\frac{8}{L^{2}} \sum_{l \neq j}\left[\frac{\sin q_{l} \sin q_{j}}{\left(\cos q_{j}-\cos q_{l}\right)^{2}}-\frac{L^{2} \rho^{2}\left(q_{j}\right)}{(l-j)^{2}}\right] .
\end{aligned}
$$

We evaluate the second term by splitting it into contributions from $\left|q_{l}-q_{j}\right|>B$ and $\left|q_{l}-q_{j}\right|<B$. The latter vanishes in the limit $B \rightarrow 0$, while the former becomes

$$
\begin{aligned}
& \frac{8}{L^{2}} \sum_{j} \sum_{|l-j|>B L \rho\left(q_{j}\right)} \frac{\sin q_{l} \sin q_{j}}{\left(\cos q_{j}-\cos q_{l}\right)^{2}}-\frac{16}{B L} \sum_{j} \rho\left(q_{j}\right)+\mathcal{O}\left(L^{-1}\right) \\
& =8 \iint_{|x-y|>B} \frac{\sin x \sin y}{(\cos x-\cos y)^{2}} \rho(x) \rho(y) d x d y-\frac{16}{B} \int d x \rho^{2}(x)+\mathcal{O}(B)+\mathcal{O}\left(L^{-1}\right) \\
& =8 \iint_{|x-y|>B} d x d y \frac{\sin y}{\cos y-\cos x} \rho^{\prime}(x) \rho(y)+\mathcal{O}(B)+\mathcal{O}\left(L^{-1}\right),
\end{aligned}
$$

where in the last line we have integrated by parts. Finally we take $B \rightarrow 0$ to obtain

$$
\Sigma_{1}=L \frac{8 \pi^{2}}{3} \int_{0}^{\pi} \rho(x)^{3} d x+8 \int d x d y \frac{\sin y}{\cos y-\cos x} \rho^{\prime}(x) \rho(y)+\mathcal{O}\left(L^{-1}\right) .
$$




\section{References}

[1] E. Lieb, T. Schultz and D. Mattis, Two soluble models of an antiferromagnetic chain, 16. 407 (1961).

[2] J.H.H. Perk, Equations of motion for the transverse correlations of the onedimensional XY-model at finite temperature, Phys. Lett. A 79,1 (1980).

[3] J.H.H. Perk, H.W. Capel, G.R.W. Quispel, and F.W. Nijhoff, Finite-temperature correlations for the Ising chain in a transverse field, Physica A 123, 1 (1984).

[4] J.H.H. Perk and H. Au-Yang, New Results for the Correlation Functions of the Ising Model and the Transverse Ising Chain, J. Stat. Phys. 135, 599 (2009).

[5] B.M. McCoy, J.H.H. Perk, and R.E. Shrock, Time-dependent correlation functions of the transverse Ising chain at the critical magnetic field, Nucl. Phys. B 220, 35 (1983).

[6] B.M. McCoy, J.H.H. Perk, and R.E. Shrock, Correlation functions of the transverse Ising chain at the critical field for large temporal and spatial separations, Nucl. Phys. B 220, 269 (1983).

[7] A. R. Its, A. G. Izergin, V. E. Korepin, and N. A. Slavnov, Temperature correlations of quantum spins, Phys. Rev. Lett. 70, 1704 (1993).

[8] F. Colomo, A. G. Izergin, V. E. Korepin and V. Tognetti, Temperature correlation functions in the XXO Heisenberg chain. I, Theor. Math. Phys 94, 11 (1993).

[9] V.E. Korepin, A.G. Izergin and N.M. Bogoliubov, Quantum Inverse Scattering Method, Correlation Functions and Algebraic Bethe Ansatz (Cambridge University Press, 1993).

[10] S. Sachdev, A. P. Young, Low Temperature Relaxational Dynamics of the Ising Chain in a Transverse Field, Phys. Rev. Lett. 78, 2220 (1997).

[11] K. Damle and S. Sachdev, Spin dynamics and transport in gapped one-dimensional Heisenberg antiferromagnets at nonzero temperatures, Phys. Rev. B 57, 8307 (1998).

[12] K. Damle, S. Sachdev, Universal Relaxational Dynamics of Gapped One-Dimensional Models in the Quantum Sine-Gordon Universality Class, Phys. Rev. Lett. 95, 187201 (2005).

[13] A. Rapp, G. Zarand, Universal diffusive decay of correlations in gapped onedimensional systems, Eur. Phys. Jour. B67, 7 (2009).

[14] A. LeClair, G. Mussardo, Finite temperature correlation functions in integrable QFT, Nucl. Phys. B 552, 624 (1999).

[15] H. Saleur, A comment on finite temperature correlations in integrable QFT, Nucl. Phys. B567 602 (2000).

[16] O.A. Castro Alvaredo and A. Fring, Finite temperature correlation functions from form factors, Nucl. Phys. B636 611 (2002).

[17] R.M. Konik, Haldane-gapped spin chains : Exact low-temperature expansion of correlation functions, Phys. Rev. B 68, 104435 (2003).

[18] B.L. Altshuler, R.M. Konik and A.M. Tsvelik, Low temperature correlation functions in integrable models: Derivation of the large distance and time asymptotics from the form factor expansion, Nucl. Phys. B739, 311 (2006).

[19] S.A. Reyes, A. Tsvelik, Phys. Rev. B 73, 220405(R) (2006).

[20] B. Pozsgay and G. Takacs, Form factors in finite volume I: form factor bootstrap and truncated conformal space, Nucl. Phys. B. $\mathbf{7 8 8} 167$ (2008).

[21] B. Pozsgay and G. Takacs, Form factors in finite volume II: Disconnected terms and finite temperature correlators, Nucl. Phys. B. 788209 (2008).

[22] F.H.L. Essler and R.M. Konik, Finite-temperature lineshapes in gapped quantum spin chains, Phys. Rev. B 78, 100403(R) (2008). 
[23] F.H.L. Essler and R.M. Konik, Finite Temperature Dynamical Correlations in Massive Integrable Quantum Field Theories, J. Stat. Mech. (2009) P09018.

[24] B. Pozsgay and G Takacs, Form factor expansion for thermal correlators, J. Stat. Mech. (2010) P11012.

[25] J. Steinberg, N.P. Armitage, F.H.L. Essler and S. Sachdev, Phys. Rev. B 99, 035156 (2019).

[26] B. Doyon, Finite-temperature form factors in the free Majorana theory, J. Stat. Mech. P11006 (2005).

[27] B. Doyon and A. Gamsa, Integral equations and long-time asymptotics for finitetemperature Ising chain correlation functions, J. Stat. Mech. P03012 (2008).

[28] M. Panfil and J.-S. Caux, Finite-temperature correlations in the Lieb-Liniger onedimensional Bose gas, Phys. Rev. A89, 033605 (2014).

[29] J. de Nardis and M. Panfil, Particle-hole pairs and density-density correlations in the Lieb-Liniger model, J. Stat. Mech. (2018) 033102.

[30] A. Cortes Cubero and M. Panfil, Thermodynamic bootstrap program for integrable QFTs: form factors and correlation functions at finite energy density., J. High Energ. Phys. 104 (2019).

[31] A. Klümper, Thermodynamics of the anisotropic spin-1/2 Heisenberg chain and related quantum chains, Z. Phys. B 91, 507 (1993).

[32] C. Destri and H.J. de Vega, Unified Approach to Thermodynamic Bethe Ansatz and Finite Size Corrections for Lattice Models and Field Theories, Nucl. Phys. B 438, 314 (1995).

[33] J. Damerau, F. Göhmann, N.P. Hasenclever and A. Klümper, Density matrices for finite segments of Heisenberg chains of arbitrary length, J. Phys. A 404439 (2007).

[34] H.E. Boos, J. Damerau, F. Göhmann, A. Klümper, J. Suzuki, and A. Weiße, Shortdistance thermal correlations in the XXZ chain, J. Stat. Mech. (2008) P08010.

[35] C. Trippe, F. Göhmann, and A. Klümper, Short-distance thermal correlations in the massive XXZ chain, Eur. Phys. J. B 73, 253 (2010).

[36] M. Dugave, F. Göhmann and K.K. Kozlowski, Low-temperature large-distance asymptotics of the transversal two-point functions of the XXZ chain, J. Stat. Mech. (2014) P04012.

[37] F. Göhmann, M. Karbach, A. Klümper, K.K. Kozlowski and J. Suzuki, Thermal form-factor approach to dynamical correlation functions of integrable lattice models, J. Stat. Mech. (2017) P113106.

[38] F. Göhmann, K.K. Kozlowski and J. Suzuki, High-temperature analysis of the transverse dynamical two-point correlation function of the $X X$ quantum-spin chain, J. Math. Phys.61, 013301 (2020).

[39] F. Göhmann, K.K. Kozlowski, J. Sirker and J. Suzuki, Equilibrium dynamics of the XX chain, Phys. Rev. B100, 155428 (2019).

[40] F. Göhmann, K.K. Kozlowski and J. Suzuki, Late-time large-distance asymptotics of the transverse correlation functions of the $X X$ chain in the space-like regime, arXiv:1908.11555.

[41] O. A. Castro-Alvaredo, B. Doyon, T. Yoshimura, Emergent hydrodynamics in integrable quantum systems out of equilibrium, Phys. Rev. X 6, 041065 (2016).

[42] B. Bertini, M. Collura, J. De Nardis, M. Fagotti, Transport in out-of-equilibrium xxz chains: Exact profiles of charges and currents, Phys. Rev. Lett. 117, 207201 (2016).

[43] B. Doyon, Exact large-scale correlations in integrable systems out of equilibrium, SciPost Phys. 5, 054 (2018).

[44] S. Gopalakrishnan, R. Vasseur and B. Ware, Anomalous relaxation and the hightemperature structure factor of XXZ spin chains, PNAS 116, 16250 (2019). 
[45] E. Barouch, B. McCoy, and M. Dresden, Statistical Mechanics of the XY Model. I, Phys. Rev. A 2, 1075 (1970).

[46] E. Barouch and B. McCoy, Statistical Mechanics of the XY Model. III, Phys. Rev. A 3, 2137 (1971).

[47] K. Sengupta, S. Powell, and S. Sachdev, Quench dynamics across quantum critical points, Phys. Rev. A 69, 053616 (2004).

[48] D. Rossini, A. Silva, G. Mussardo, and G.E. Santoro, Effective Thermal Dynamics Following a Quantum Quench in a Spin Chain, Phys. Rev. Lett. 102, 127204 (2009).

[49] D. Rossini, S. Suzuki, G. Mussardo, G.E. Santoro, and A. Silva, Long time dynamics following a quench in an integrable quantum spin chain: Local versus nonlocal operators and effective thermal behavior, Phys. Rev. B 82, 144302 (2010).

[50] P. Calabrese, F.H.L. Essler, and M. Fagotti, Quantum Quench in the Transverse-Field Ising Chain, Phys. Rev. Lett. 106, 227203 (2011).

[51] P. Calabrese, F.H.L. Essler, and M. Fagotti, Quantum quench in the transverse field Ising chain: I. Time evolution of order parameter correlators. J. Stat. Mech. (2012) P07016.

[52] P. Calabrese, F.H.L. Essler, and M. Fagotti, Quantum Quench in the Transverse Field Ising Chain II: Stationary State Properties, J. Stat. Mech. (2012) P07022.

[53] M. Fagotti and F.H.L. Essler, Reduced density matrix after a quantum quench, Phys. Rev. B 87, 245107 (2013).

[54] D. Schuricht and F.H.L. Essler, Dynamics in the Ising field theory after a quantum quench, J. Stat. Mech. (2012) P04017.

[55] F.H.L. Essler, S. Evangelisti and M. Fagotti, Dynamical Correlations After a Quantum Quench, Phys. Rev. Lett. 109, 247206 (2012).

[56] M. Kormos, M. Collura and P. Calabrese, Analytic results for a quantum quench from free to hard-core one-dimensional bosons, Phys. Rev. A 89, 013609 (2014).

[57] J. De Nardis and J.-S. Caux, Analytical expression for a post-quench time evolution of the one-body density matrix of one-dimensional hard-core bosons, J. Stat. Mech. (2014) P12012.

[58] B. Bertini, D. Schuricht, and F.H.L. Essler, Quantum quench in the sine-Gordon model, J. Stat. Mech. (2014) P10035.

[59] A. Cortés Cubero and D. Schuricht, Quantum quench in the attractive regime of the sine-Gordon model, J. Stat. Mech. (2017) 103106.

[60] D. X. Horvath, M. Kormos and G. Takacs, Overlap singularity and time evolution in integrable quantum field theory, JHEP 08 (2018) 170.

[61] F. Iglói and H. Rieger, Quantum Relaxation after a Quench in Systems with Boundaries, Phys. Rev. Lett. 106, 035701 (2011).

[62] H. Rieger and F. Iglói, Semiclassical theory for quantum quenches in finite transverse Ising chains, Phys. Rev. B 84, 165117 (2011).

[63] B. Blass, H. Rieger, and F. Iglói, Quantum relaxation and finite-size effects in the XY chain in a transverse field after global quenches, EPL 9930004 (2012).

[64] S. Evangelisti, Semi-classical theory for quantum quenches in the O(3) non-linear sigma model, J. Stat. Mech. (2013) P04003.

[65] M. Kormos and G. Zaránd, Quantum quenches in the sine-Gordon model: a semiclassical approach, Phys. Rev. E 93, 062101 (2016).

[66] J.-S. Caux and F.H.L. Essler, Time Evolution of Local Observables After Quenching to an Integrable Model, Phys. Rev. Lett. 110, 257203 (2013).

[67] E. Granet, J. L. Jacobsen and H. Saleur, Analytical results on the Heisenberg spin chain in a magnetic field, J. Phys. A: Math. Theor. 52, 255302 (2019). 
[68] Z. Ristivojevic, Excitation spectrum of the Lieb-Liniger model, Phys. Rev. Lett. 113 , 015301 (2014).

[69] A. Leclair, F. Lesage, S. Sachdev and H. Saleur, Finite temperature correlations in the one-dimensional quantum Ising model, Nucl. Phys. B 482, 579 (1996).

[70] B. Wouters, J. De Nardis, M. Brockmann, D. Fioretto, M. Rigol, and J.-S. Caux, Quenching the Anisotropic Heisenberg Chain: Exact Solution and Generalized Gibbs Ensemble Predictions, Phys. Rev. Lett. 113,117202 (2014).

[71] M. Brockmann, B. Wouters, D. Fioretto, J. De Nardis, R. Vlijm and J.-S. Caux, Quench action approach for releasing the Néel state into the spin-1/2 XXZ chain, Stat. Mech. (2014) P12009.

[72] B. Pozsgay, M. Mestyán, M.A. Werner, M. Kormos, G. Zaránd, and G. Takács, Correlations after Quantum Quenches in the XXZ Spin Chain: Failure of the Generalized Gibbs Ensemble, Phys. Rev. Lett. 113, 117203 (2014).

[73] M. Mestyán, B. Pozsgay, G. Takács, and M.A. Werner, Quenching the XXZ spin chain: quench action approach versus generalized Gibbs ensemble, J. Stat. Mech. (2015) P04001.

[74] J. De Nardis, B. Wouters, M. Brockmann, and J.-S. Caux, Solution for an interaction quench in the Lieb-Liniger Bose gas, Phys. Rev. A 89, 033601 (2014).

[75] L. Piroli, P. Calabrese, and F.H.L. Essler, Multiparticle Bound-State Formation following a Quantum Quench to the One-Dimensional Bose Gas with Attractive Interactions, Phys. Rev. Lett. 116, 070408 (2016).

[76] J. De Nardis, L. Piroli and J.-S. Caux, Relaxation dynamics of local observables in integrable systems, Phys. A: Math. Theor. 48 43FT01 (2015).

[77] S. Lukyanov, Free Field Representation For Massive Integrable Models, Commun. Math. Phys. 167, 183 (1995).

[78] F. A. Smirnov, Form factors in completely integrable models of quantum field theory (World Scientific, Singapore, 1992).

[79] S. Sachdev, Quantum Phase Transitions, Cambridge University Press, 2001.

[80] A. Bugrij, Correlation function of the two-dimensional Ising model on the finite lattice. I, Theor. Math. Phys. 127, 528 (2001).

[81] A. Bugrij and O. Lisovyy, Spin matrix elements in 2D Ising model on the finite lattice, Phys. Lett. A 319, 390 (2003).

[82] G. von Gehlen, N. Iorgov, S. Pakuliak, V. Shadura and Y. Tykhyy, Form-factors in the Baxter-Bazhanov-Stroganov model II: Ising model on the finite lattice, J. Phys. $\mathbf{A}$ 41, 095003 (2008).

[83] N. Iorgov, V. Shadura and Yu. Tykhyy, Spin operator matrix elements in the quantum Ising chain: fermion approach, J. Stat. Mech. (2011) P02028.

[84] O. Derzhko and T. Krokhmalskii, Dynamic structure factor of the spin-1/2 transverse Ising chain, Phys. Rev. B, 56, 11659, (1997). 